\title{
A revised classification of the sister tribes Palicoureeae and Psychotrieae (Rubiaceae) indicates genus-specific alkaloid accumulation
}

\author{
Andreas Berger (1D) Karin Valant-Vetschera $(\mathbb{D}) \cdot$ Johann Schinnerl $\mathbb{D}$ - \\ Lothar Brecker (i)
}

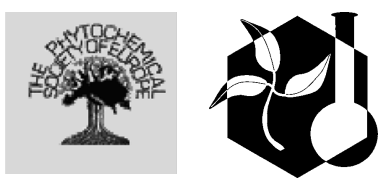

Received: 26 December 2020/ Accepted: 17 July 2021 / Published online: 17 September 2021

(C) The Author(s) 2021

\begin{abstract}
Tribes Palicoureeae and Psychotrieae (Rubiaceae, Gentianales) are complex and speciose sister groups with a pantropical distribution. Since the initial studies on ipecacuanha more than two centuries ago, species of the group have been subject to numerous phytochemical studies yielding diverse specialized ("secondary") metabolites, most of them alkaloids. However, the generic limits within the tribes have long been unclear and only recently, monophyletic genera have been delimited and segregated from a once broadly circumscribed Psychotria. Thus, a phylogenybased and taxonomically updated review of phytochemical literature was performed which allowed assigning the bulk of phytochemical data previously reported for Psychotria to various segregate genera such as Carapichea, Eumachia and Palicourea. This review not only challenges the common perception of Psychotria as a monoterpene-indole alkaloid-rich
\end{abstract}

A. Berger $(\varangle) \cdot$ K. Valant-Vetschera $\cdot$ J. Schinnerl Department of Botany and Biodiversity Research, University of Vienna, Rennweg 14, 1030 Vienna, Austria e-mail: andi.berger@univie.ac.at

L. Brecker $(\bowtie)$

Department of Organic Chemistry, University of Vienna, Währinger Strasse 38, 1090 Vienna, Austria

e-mail: lothar.brecker@univie.ac.at genus. It also highlights that each of its relatives differs by accumulating specific groups of alkaloids, which is of major importance for understanding animal-plant interactions such as herbivory, as well as for drug discovery. The alkaloid complement of each of these genera is here enumerated and discussed, which should provide a framework for future studies addressing the biosynthesis, evolution, ecological and pharmacological significance of specialized metabolite differentiation in this abundant, ecologically and ethnopharmacologically important group.

Keywords Palicoureeae - Psychotrieae · Chemosystematics $\cdot$ Secondary metabolites . Alkaloids
Abbreviations
DMT $N, N$-Dimethyltryptamine
IA Indole alkaloid
MAO Monoamine oxidase
MIA Monoterpene-indole alkaloid
PA Protoalkaloid
PIA Polypyrroloindoline alkaloid
PSR Pictet-Spengler reaction
SGD Strictosidine B-glucosidase
STR Strictosidine synthase
T5H Tryptamine 5-hydroxylase
TA Tryptamine analogue
TIQA Tetrahydroisoquinoline alkaloid
$\beta C A \quad \beta$-Carboline alkaloid 


\section{Introduction}

Taxonomy of Palicoureeae and Psychotrieae

The Psychotria alliance is a speciose and complex group of more than 3100 species, now classified in two sister tribes Palicoureeae and Psychotrieae within the coffee family (Rubiaceae, Gentianales; Nepokroeff et al. 1999; Razafimandimbison et al. 2014; Robbrecht and Manen 2006). Most of the species included here are shrubs and understory treelets, but other growth forms are also occasionally found. They contribute a significant part to rainforest understory species diversity, abundance and biomass (Gentry 1990), and provide an important food source for frugivorous birds (Krebber et al., in prep.; Snow 1981). Furthermore, many species are of ethnobotanical importance (e.g. Rivier and Lindgren 1972) and have proven to be a rich source of various classes of alkaloids (e.g. Calixto et al. 2016; de Carvalho Junior et al. 2017; Martins and Nunez 2015; Porto et al. 2009; Yang et al. 2016).

Traditionally, an overly broad generic concept was applied in the classification of the group, which resulted in lumping most species under the large and polyphyletic genus Psychotria (e.g. Steyermark 1972). Recent DNA-phylogenetic studies and a reevaluation of morphological characters have radically challenged the traditional circumscription of Psychotria, the largest genus of the alliance and one of the largest genera of flowering plants (e.g. Nepokroeff et al. 1999; Razafimandimbison et al. 2014; Robbrecht and Manen 2006). As a result, views shifted towards a narrower concept of Psychotria and Psychotrieae that peaked in the establishment of the sister tribe Palicoureeae and the ongoing transfer of hundreds of species of Psychotria subg. Heteropsychotria to other genera. The new generic circumscription renders all the genera monophyletic groups, and is now widely accepted in floristic and systematic literature (e.g. Lorence and Taylor 2012; Kiehn and Berger 2020; Taylor 2014). The entire group is particularly diverse in the Neotropics, where it includes the genera Psychotria (tribe Psychotrieae), as well as Carapichea, Eumachia, Geophila, Notopleura, Palicourea and Rudgea (tribe Palicoureeae). Phylogenetic relationships among the genera are shown in a cladogram in Fig. 1.
The genera Geophila and Rudgea have long been recognized and their generic circumscription remained rather stable over time. The genera Carapichea, Notopleura and Eumachia are more problematic with respect to delimitation, but the corresponding species of these lineages have already been identified (Taylor and Gereau 2013; Taylor et al. 2017; Taylor 2001, 2005). In order to render both Palicourea and Psychotria monophyletic groups, all species of Psychotria subg. Heteropsychotria have to be transferred to Palicourea, the oldest available name for the genus. Most of these combinations have already been provided in a number of recent publications (Berger 2017, 2018b; Borhidi 2011, 2017; Delprete and Kirkbride 2016; Delprete and Lachenaud 2018; Taylor and Hollowell 2016; Taylor et al. 2010; Taylor 2015a, b, 2017, 2018, 2019a, b), but many species still lack a formal name under Palicourea pending future studies.

Given the taxonomic complexity and recent changes in the generic placement of many species, the problem that metabolites reported from Psychotria were actually isolated from species now assigned to other genera became apparent. Consequently, it appears necessary to review data on specialized metabolite accumulation of the whole group and assign the correct generic identity to each of the previously studied species. This approach allows the re-interpretation of phytochemical data and alkaloid accumulation patterns in a phylogenetic context. Ultimately, this helps to understand the evolution of plant metabolites and their biosynthetic relationships, and is of major importance in drug discovery and for shaping animal-plant interactions such as herbivory.

\section{Alkaloids of Palicoureeae and Psychotrieae}

Species of tribes Palicoureeae and Psychotrieae are a rich source of structurally diverse alkaloids (e.g., Bernhard et al. 2011; Berger et al. 2012, 2015a, 2017; Kornpointner et al. 2018, 2020; Lopes et al. 2004; Schinnerl et al. 2012). Further described compound groups include cyclotides (Koehbach et al. 2013), flavonoids and other polyphenols (Berger et al. 2016) and iridoids (Berger 2012; Lopes et al. 2004), highlighting the chemical diversity of the tribes. Alkaloids are particularly diverse in the genus Palicourea lending the group to a more in-depth analysis 
(a)

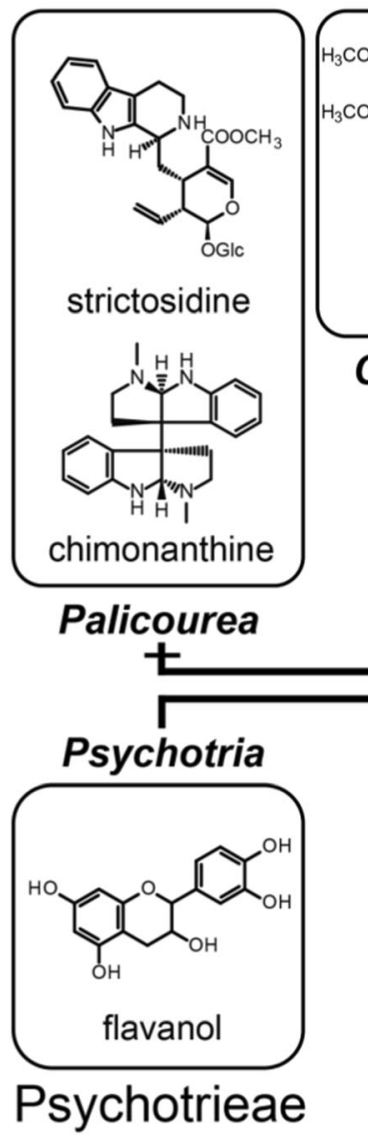

Fig. 1 Major classes of specialized metabolites characterising the genera of the sister tribes Palicoureeae (all genera except Psychotria) and Psychotrieae (Psychotria) plotted on a simplified phylogeny of the group. (a) Boxes with representative structures illustrate the compound groups characterizing each genus. For alkaloid containing lineages, rare compound classes found in less than $15 \%$ of the surveyed species are not shown for

(b)
Hymenocoleus + Puffia

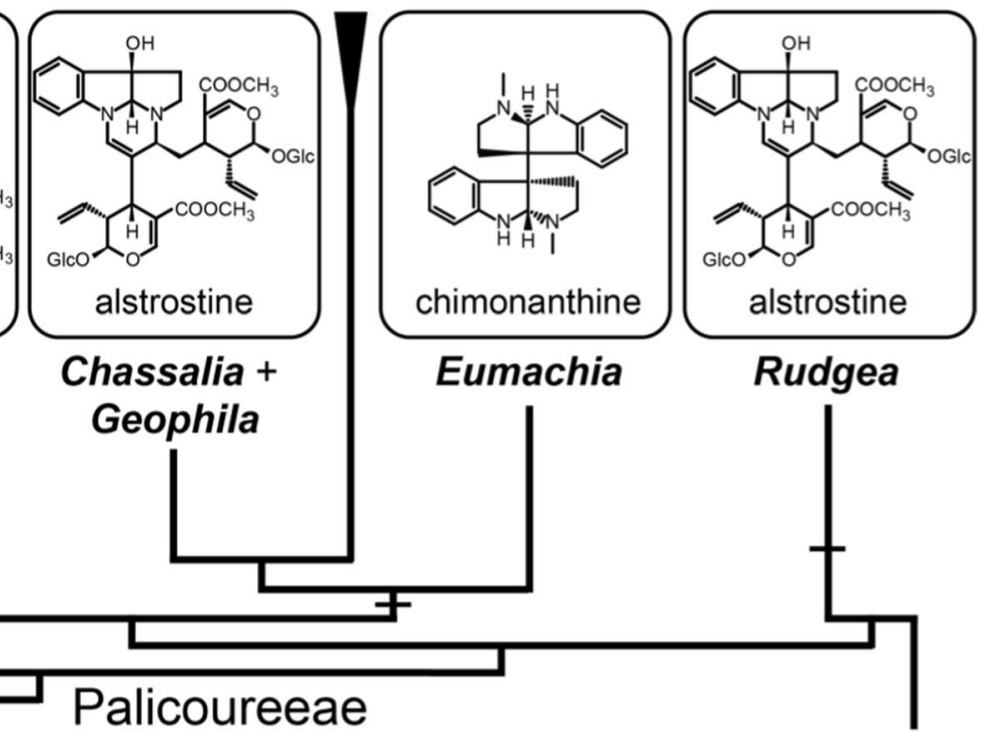

$\neq$ tyrosine tryptophan<smiles>NC(Cc1c[nH]c2ccccc12)C(=O)O</smiles>

(typhan

matters of clarity. (b) In alkaloid-accumulating clades, crossbars on the phylogenetic tree indicate the amino acid building blocks incorporated in the alkaloids of the respective genera. Note that many species and genera have at times been included in a broadly circumscribed Psychotria, and their compounds have likewise been ascribed to the genus

molecular structures, the compounds can only hardly be differentiated from other natural product classes. Furthermore, it is difficult to divide them into subgroups according to chemical structure. To basically divide the alkaloid discussed here into subgroups, we refer to the divisions in "true alkaloids" and "protoalkaloids" used, e.g., by Aniszewski (2015). As this division is not always unambiguous, we use these terms in quotation marks.

The bulk of alkaloids of Palicoureeae and Psychotrieae-as well as in general-are "true alkaloids" containing one or more amino acid-derived nitrogen atoms which are part of a heterocycle. By contrast sustainable definition of alkaloids, which is based on 
"protoalkaloids" lack such a nitrogen-containing heterocycle (Aniszewski 2015). One of the largest and most important groups of "true alkaloids" are indole alkaloids (IA) which originate from the amino acid tryptophan and its decarboxylation product tryptamine bearing the nominate indole scaffold. This group includes simple compounds such as serotonin and harmine, but it is better known for the complex and structurally diverse monoterpene-indole alkaloids (MIA). More than 5,100 derivatives are known (Cordell et al. 2001), and all of these are formed by a stereospecific strictosidine synthase (STR)-catalysed Pictet-Spengler reaction (PSR) between the amine function of tryptamine, the decarboxylation product of tryptophan, and the aldehyde function of secologanin, a seco-iridoid derived from non-mevalonate terpene biosynthesis (Aniszewski 2015; O'Connor and Maresh 2006).

\section{Generic affiliation of phytochemically studied species}

During our studies on tribes Palicoureeae and Psychotrieae an extensive literature survey yielded a presumably complete list of phytochemical publications. A combination of extensive fieldwork by two of the authors (AB, JS), herbarium studies in the herbaria CR, W and WU (e.g. Berger 2018a, b) and the consultation of recent taxonomic revisions (e.g. Berger 2017, 2018b; Borhidi 2011, 2017; Delprete and Kirkbride 2016; Delprete and Lachenaud 2018; Lorence and Taylor 2012; Taylor and Gereau 2013; Taylor and Hollowell 2016; Taylor et al. 2010; Taylor 2001, 2005, 2014, 2015a, b, 2017, 2018, 2019a, b), TROPICOS (https://www.tropicos.org) and other relevant databases (e.g. JACQ, http://jacq.org; POWO, http://www.plantsoftheworldonline.org) subsequently allowed assessing the generic placement of the studied species based on the currently accepted phylogenetic framework (Nepokroeff et al. 1999; Razafimandimbison et al. 2014; Robbrecht and Manen 2006). Using a modern generic circumscription that renders the genera monophyletic groups finally allows reviewing specialized metabolites in an evolutionary context.

A total of 180 phytochemical publications were retrieved and evaluated in the present study. Species merely reported as alkaloid-positive on basis of TLCanalyses with alkaloid-sensitive Dragendorff's reagent or similar analyses, as well as species accumulating monofluoroacetate (Cook et al. 2014; de L Carvalho et al. 2016) are not considered in the present work. In addition, one study was excluded due to unclear taxonomic affinity of the studied material, even at the tribal level (Sandra et al. 2018, appendix, Table 11). As currently circumscribed (see above) the remaining 179 studies refer to eight genera and 102 species, if two unidentified taxa of Psychotria are considered as separate species.

Table 1 provides an overview on the current state of phytochemical research within the ten genera currently assigned to Palicoureeae and Psychotrieae. Most studies pertain on species of Palicoureeae, and Palicourea is the best studied genus of the tribe. Furthermore, the data shows gaps in knowledge and pinpoints to some groups remaining underrepresented or unstudied. Table 11 (see appendix) provides a referenced and taxonomically updated compilation of all 102 phytochemically-studied species and their alkaloid content. The list is arranged by accepted names, but also includes synonyms if they have been used in the original publications. Compounds from other biosynthetic groups (coumarins, flavonoids etc.) are not individually mentioned and subsumed under their compound groups.

\section{Phytochemical differentiation of genera}

As the most-significant result of the present analysis we show that tribes Palicoureeae and Psychotrieae as well as the respective genera are chemically distinct and each is characterized by a specific blend of metabolites. Briefly, Psychotrieae and Psychotria are largely characterized by polyphenols and tannin accumulation, with polypyrroloindoline type IA reported from a couple of Asian and Pacific species. MIA are here shown to be absent in the genus instead being restricted to the tribe Palicoureeae. As such the here-elaborated phytochemical view of the group is in strong contrast to previous analyses listing MIA as specific for Psychotria (Calixto et al. 2016; de Carvalho Junior et al. 2017; Martins and Nunez 2015; Yang et al. 2016).

In the tribe Palicoureeae the phytochemical situation is more diverse, and most lineages are capable of biosynthesising IA and/or MIA. Palicourea largely accumulates strictosidine type MIA with few species 
Table 1 Accepted genera included in tribes Palicoureeae and Psychotrieae, some of the more frequently used synonyms, and the current state of knowledge of their phytochemistry

\begin{tabular}{|c|c|c|c|c|c|}
\hline Accepted genus & Tribe & Selected synonyms & Spp. no. ${ }^{\mathrm{a}}$ & Studied & $\%$ studied \\
\hline Carapichea & Palicoureeae & Ipecacuanha & 23 & 3 & 13.0 \\
\hline Chassalia & Palicoureeae & & 140 & 6 & 4.3 \\
\hline Eumachia & Palicoureeae & Chazaliella, Margaritopsis & 83 & 10 & 12.0 \\
\hline Geophila & Palicoureeae & & 24 & 2 & 8.3 \\
\hline Hymenocoleus & Palicoureeae & & 13 & 0 & 0.0 \\
\hline Notopleura & Palicoureeae & Psychotria sect. Notopleura & 210 & 3 & 1.4 \\
\hline Palicourea & Palicoureeae & Cephaelis, Psychotria subg. Heteropsychotria & 800 & 49 & 6.1 \\
\hline Puffia & Palicoureeae & & 1 & 0 & 0.0 \\
\hline Rudgea & Palicoureeae & & 200 & 3 & 1.5 \\
\hline Psychotria & Psychotrieae & Grumilea, Hydnophytum, Mapouria & 1600 & 47 & 2.9 \\
\hline
\end{tabular}

${ }^{a}$ Species numbers according to Razafimandimbison et al. (2014), only Eumachia according to Taylor et al. (2017). Spp.: species

forming other classes of IA and/or MIA. The genera Chassalia, Geophila and Rudgea are characterized by alstrostine-type MIA. The genus Carapichea forms tetrahydroisoquinoline alkaloids (TIQA) based on tyrosine-derived dopamine and secologanin. Finally, the genus Notopleura is devoid of all of these alkaloids, instead accumulating various types of quinones. No data is currently available for the African genera Hymenocoleus and the Malagasy endemic monotypic Puffia. Minor exceptions in the retrieved patterns include a few species in alkaloidaccumulating clades that have probably lost the ability to form alkaloids. In such cases, ubiquitous iridoids or polyphenols such as flavonoids and chlorogenic acids often replace these (e.g. Benevides et al. 2005, Berger et al. 2016; Sosa Moreno 2011).

As a word of caution, however, the absence of a report of a certain class of compounds does not necessarily imply that they don't exist in a given plant species. Probable reasons are seasonal or regional chemical and/or genetic differentiations (e.g. Berger et al. 2015; de Sousa Queiroz et al. 2011), targeted isolation efforts (e.g. bioactivityguided fractionation, acid-base extraction) or other sampling or methodological issues. Most phytochemical publications were focussed on the isolation of putatively bioactive alkaloids as evidenced by the frequent use of acid-base extraction. We therefore expect less bias against alkaloids when compared to other compound classes that have not been in focus, facilitating the chemosystematic interpretation presented here.
In order to illustrate the phytochemical differentiation of the genera, the obtained metabolite groups were plotted on a phylogeny (Razafimandimbison et al. 2014) showing relationships within the genera of tribes Palicoureeae and Psychotrieae (Fig. 1). Furthermore, the biosynthetic origin of the nitrogen atom from either of the amino acids tyrosine (in Carapichea) of tryptophan (other genera) are indicated by crossbars on the tree. In the present study a brief taxonomic introduction is given for each genus and the respective alkaloids are grouped and enumerated according to structural similarity and putative biosynthetic relationships (see also Berger et al. 2021).

\section{Palicoureeae}

\section{Carapichea Aubl.}

The neotropical genus Carapichea (Palicoureeae) comprises of about 23 species of shrubs and treelets distributed from Nicaragua south to Bolivia and eastern Brazil, and its species were long included in Psychotria. The genus features great morphological diversity which makes it difficult to diagnose (Taylor and Gereau 2013): Dried leaves grayish green to brownish; stipules persistent-marcescent, entire, lobed or laciniate, with margins fragmenting with age; inflorescences terminal, (sub)capitate glomerulate or branched, green, white to purple, sessile to pedunculated, bracts well-developed to reduced, the outermost sometimes involucral; corolla straight, tubular to 
funnelform, white, yellow, orange to purple; fruit colour ranging from white, red, blue to black; pyrenes with a smooth, 1-crested or 3-5-ridged dorsal side, and a plane or grooved ventral side, opening by a single basal ventral, or 3-4 dorsal preformed germination slits along ridges. According to molecular phylogenetic data, the genus is well-supported as sister to a clade containing Chassalia, Eumachia, Geophila, Hymenocoleus and Puffia (Andersson 2002; Razafimandimbison et al. 2014; see Fig. 1).

The genus Carapichea is the well-known source of structurally unique and pharmacologically important ipecac alkaloids otherwise known only from the unrelated genus Alangium (Cornaceae, Cornales). They were discovered more than two centuries ago in the historically important medicinal plant ipecacuanha, which is also known as the vomiting root. Since that time, the drug has been widely used for the induction of vomiting as well as for the treatment of amoebic dysentery (e.g. Lee 2008). The drug is derived from the roots of Carapichea ipecacuanha (Brot.) L. Andersson, a species previously confused with the chemically distinct Ronabea emetica (L. f.) A. Rich. containing asperuloside and other iridoids (Berger et al. 2011). Although the use of the vomiting root and its products has decreased due to severe side effects, some derivatives are currently under consideration as possible leads for the discovery of anticancer drugs (Uzor 2016; Akinboye et al. 2017). The emetic and antiamoebic effects of the drug are largely related to the major alkaloids emetine and cephaeline, possessing a monoterpenoid tetrahydroisoquinoline skeleton. In addition, many other alkaloids are present in minor quantities (e.g. Garcia et al. 2005; Hatfield et al. 1981).

Biosynthesis of ipecac alkaloids has been comparably well-studied and starts with a stereospecific Pictet-Spengler condensation of tyrosine-derived dopamine and secologanin in a similar way as in strictosidine. The corresponding enzyme $N$-deacetyl isoipecoside synthase forms the $1 \alpha$ epimer whereas $N$ deacetyl ipecoside synthase forms the respective $1 \beta$ epimer. The latter $1 \beta$ - $N$-deacetyl ipecoside is subject to various reactions such as $O$-methylation, $N$-acetylation or lactam formation leading to ipecoside, alangiside and related alkaloids (Nomura et al. 2008; Nomura and Kutchan 2010a, b; see Fig. 2).

By contrast, deglucosylation of $1 \alpha-N$-deacetyl isoipecoside by the enzyme ipecac alkaloid $\beta$-D- glucosidase (Ipeglu1) leads to an aglycon which is further processed to protoemetine. Finally, protoemetine is condensed with a second dopamine unit leading to cephaeline, emetine and related alkaloids (Nomura et al. 2008; see Fig. 3). Both groups are subject to various $O$-methylations by three dedicated ipecac alkaloid $O$-methyltransferases creating much of the observed structural diversity (Nomura and Kutchan 2010a). Finally, biosynthesis involves complex subcellular compartmentation between cytosol and vacuole (Nomura and Kutchan 2010b).

In addition to Carapichea ipecacuanha, ipecac alkaloids were isolated from Carapichea affinis (Standl.) L. Andersson (Bernhard et al. 2011; Kornpointner et al. 2018) and Carapichea klugii (Standl.) C.M. Taylor (Muhammad et al. 2003, as Psychotria klugii Standl.). Shamma (1972) and Wiegrebe et al. (1984) also report ipecac alkaloids from what they called "Psychotria granadensis Benth." However, they confused the latter name with Uragoga granatensis Baill., a name that lacks a respective combination under the genus Psychotria and is a synonym of Carapichea ipecacuanha. In turn, Psychotria granadensis Benth. is a synonym of Psychotria nervosa Sw. which lacks alkaloids instead accumulating tannins (Berger 2012). Table 11 (appendix) lists all alkaloids isolated from species of Carapichea and the corresponding structures are found in Figs. 2 and 3. Besides ipecac alkaloids, two iridoids glucosides were also isolated from the genus (Itoh et al. 1991).

Chassalia Comm. ex Poir.

Chassalia (Palicoureeae) is a paleotropical genus found in Africa, Asia, and the West Indian Ocean region. It includes ca. 140 species of shrubs and treelets, although a few species are epiphytic or lianescent. The genus is largely diagnosed by indurated and persistent stipules; fleshy-succulent, white or brightly coloured inflorescence axes; often winged flower buds, long-tubed and slightly curved corollas; and pyrenes possessing a large ventral excavation, as well as a dorsal, basal, median preformed germination slit. Chassalia is paraphyletic with respect to Geophila and comprises of three clades: the basal Southeast Asian 'Chassalia sp.ck25', the small 'East African Chassalia clade' and Chassalia s. str. The former two clades have to be recognized at the generic level, if the morphologically 


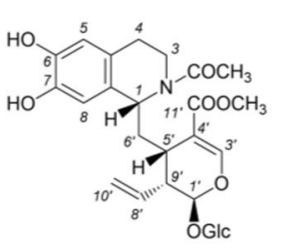

ipecoside

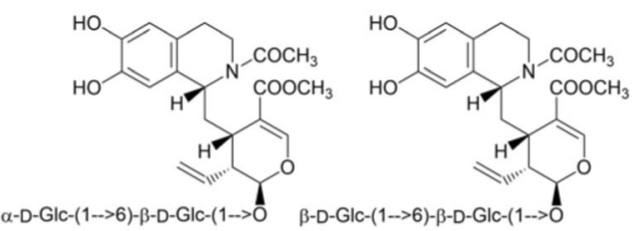

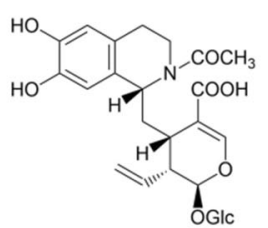

ipecosidic acid<smiles>C=C[C@H]1[C@@H](OC(C)=O)OC=C(O)[C@H]1[C@H]1c2cc(O)c(O)cc2CCN1C(C)=O</smiles>

6-O-methyl ipecoside

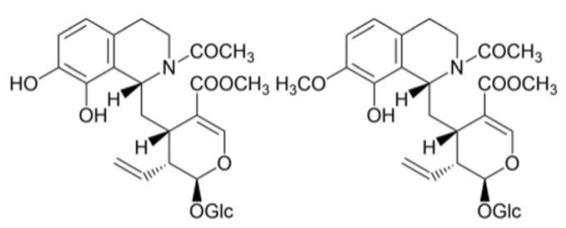

neoipecoside

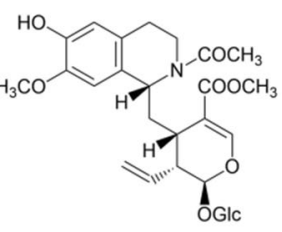

7-0-methyl ipecoside

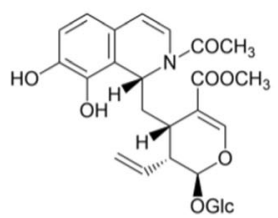

3,4-dehydro neoipecoside

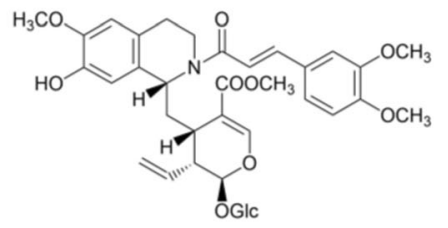

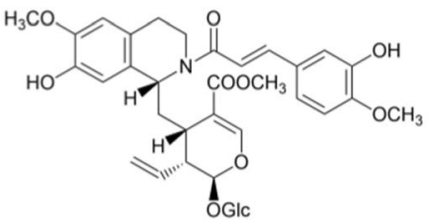

borucoside

3"'-O-demethyl borucoside<smiles>C=C[C@@H]1c2cc(O)c(O)cc2CCN(C(=O)/C=C/c2ccc(O)c(OC)c2)[C@H]1C[C@H]1C(C(=O)OC)=CO[C@H](Cl)[C@@H]1OC(C)=O</smiles>

trans-cephaeloside

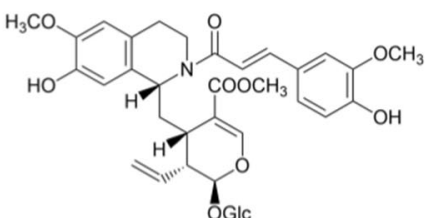

6-O-methyl trans-cephaeloside

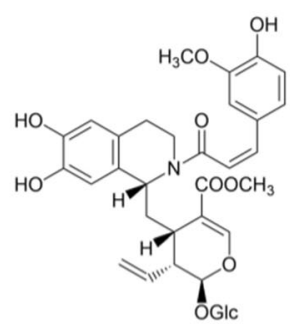

cis-cephaeloside

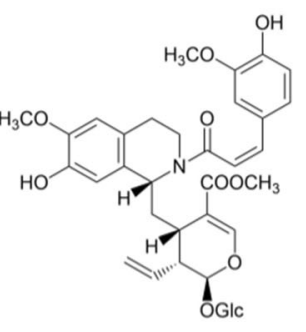

6-O-methyl cis-cephaeloside<smiles>C=C[C@@H]1[C@H](OC(C)=O)OC=C2C(=O)N3CCc4cc(OC)c(O)cc4[C@@H]3C[C@@H]21</smiles>

alangiside<smiles>C=C[C@@H]1[C@@H]2C[C@@H]3c4cc(O)c(O)cc4CCN3C(=O)C2=CO[C@@H]1OC(C)=O</smiles>

demethyl alangiside

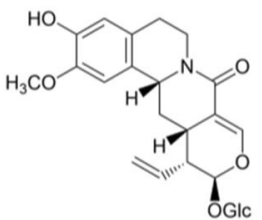

3-O-demethyl-2-O-methyl alangiside<smiles>C=CC1C(O)OC=C2C(=O)N3CCc4cc(O)c(OC)cc4[C@H]3[C@H]2C1O</smiles>

2-O- $\beta$-D-glucopyranosyl-demethyl alangiside

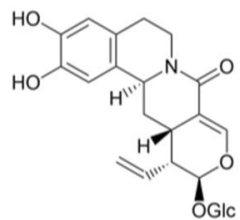

demethyl isoalangiside<smiles>C=C[C@H](CO)[C@H]1CCN2CCc3cc(OC)c(OC)cc3[C@H]2C1</smiles>

7-O-methyl alangine

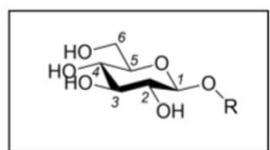

$$
\text { он }
$$

Fig. 2 Ipecac alkaloids isolated from Carapichea species, I. Most of the alkaloids belonging to the biosynthetic group of ipecosides, alangines and related compounds show a glucose moiety in $\beta$ configuration originating from the secologanin moiety. The structure of the glucoside is shown in a frame at the bottom of the figure, and represents all "Glc" units indicated in this and other figures of the present article. The numbering of the positions of ipecoside and compounds in other figures shows the most commonly used numbering schemes of the respective substance classes. These, however, do not necessarily relate to the numbering of the IUPAC names of the corresponding compounds. The numbering of ipecoside is based on Itoh et al. (1989), but other numbering schemes are also used (e.g. Bernhard et al. 2011; see supplementary figure S1) 


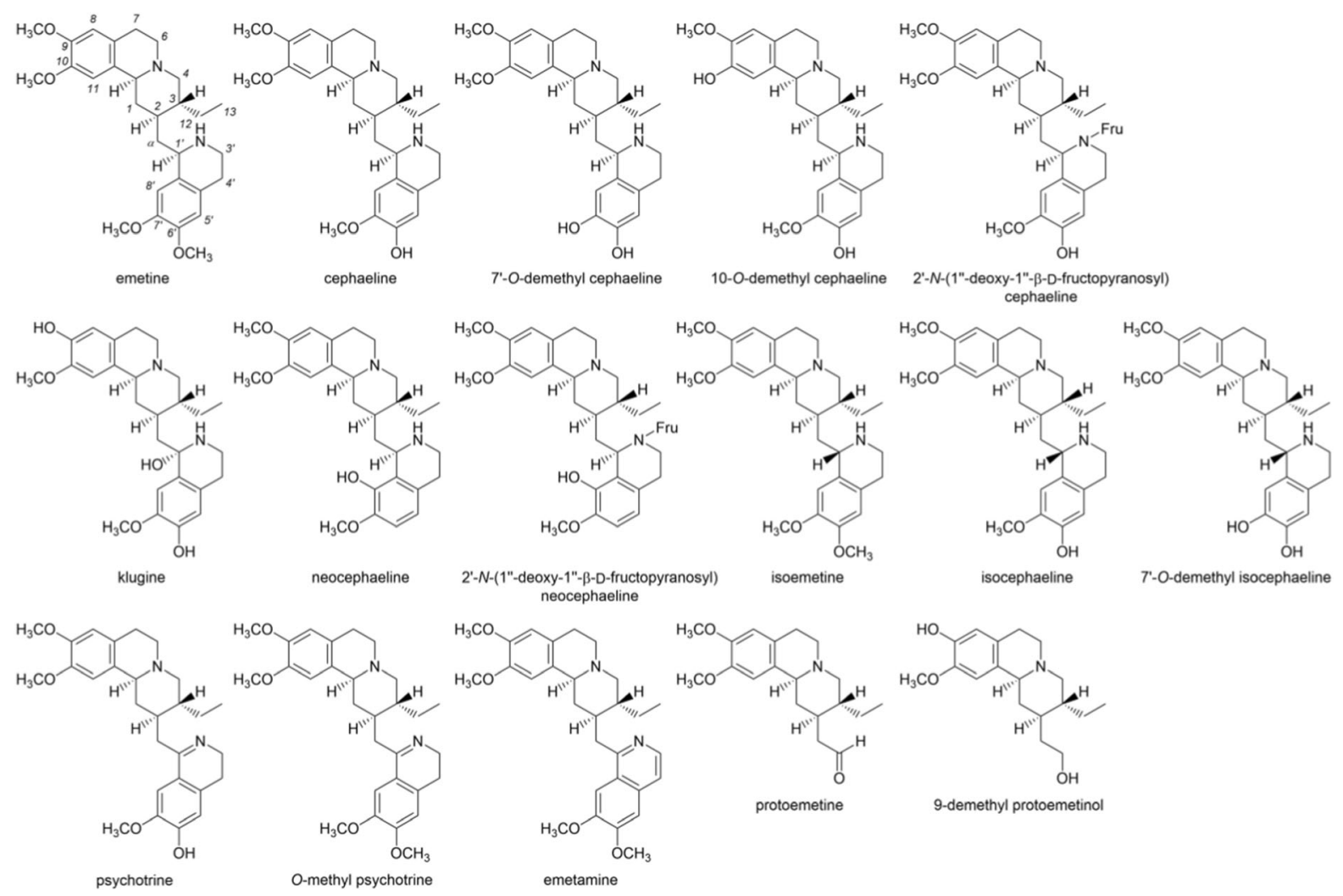

Fig. 3 Ipecac alkaloids isolated from Carapichea species, II. The biosynthetic group of protoemetine, emetine and related alkaloids showing a $1 \alpha$ configuration. With two exceptions these are aglycones containing two dopamine units. The numbering of

emetine is based on Shamma (1972), but other numbering schemes are also used (e.g. Bernhard et al. 2011; Uzor 2016; see supplementary figure $\mathrm{S} 1$ )

et al. 2012; Kornpointner et al. 2020). As such alstrostines have a peculiar distribution being both widespread but uncommon in Palicoureeae.

\section{Eumachia DC.}

The pantropical genus Eumachia (Palicoureeae) has a long and confusing taxonomic and nomenclatural history, and includes more than 83 species found in the Neotropics, Africa, Asia and the Pacific region. Most of its species were initially placed in a broadly defined Psychotria although taxa showing aberrant morphological features have long been separated at the generic level. For example, African species were named Chazaliella E.M.A. Petit \& Verdc. and a number of sclerophyllous Cuban and Hispaniolan endemics with spiny leaf tips were named Margaritopsis $\mathrm{C}$. Wright. Phylogenetic studies indicated that all form a well-supported clade, initially named 
Margaritopsis (Taylor 2005). Finally, it was shown that Eumachia-previously applied to a single species endemic to Fiji, Tonga and Samoa-is the oldest available name for the group and therefore has nomenclatural priority (Barrabé and Davis 2013; Barrabé et al. 2012; Taylor et al. 2017). The genus is well-supported as sister to a clade containing Chassalia, Geophila, Hymenocoleus and Puffia (Razafimandimbison et al. 2014).

Species of Eumachia are rather poor in diagnostic characters but are recognized by: A shrubby habit; frequently flattened and longitudinally ridged internodes; persistent stipules which become indurate and fragmented with age and sometimes show glandular appendages; leaves drying greyish or pale yellowish greenish; terminal inflorescences with green to whitish axes; actinomorphic, white, creamy to yellow-green corollas with straight base; orange to red fruits; pyrenes hemispherical in cross-section, without ventral groove or intrusion and with two basal ventral marginal preformed germination slits; seeds lacking a red ethanol-soluble seed-coat pigment; endosperm not ruminate, and frequently with small inner central ventral invagination (Barrabé et al. 2012; Delprete and Kirkbride 2015; Razafimandimbison et al. 2014; Taylor 2005; Taylor et al. 2017).

To date, nine species of Eumachia were studied phytochemically. Most originate from Asia, Australasia and the Pacific region, with a single neotropical species yet studied (see Table 2). Species of the genus accumulate a group of IA known as polypyrroloindoline alkaloids, but these are also referred to as cyclotryptamine, cis-pyrrolidino[2,3$b$ ]indoline or hexahydropyrrolo indole alkaloids
(Jamison et al. 2017). They consist of two or more monomers connected by two or more quaternary carbon stereocenters that allow for a great diversity of stereoisomers, some of which have not been definitely assigned (but see Jannic et al. 1999). Most of the studied species accumulate dimers such as (+)chimonanthine and (-)-calycanthine, but oligomers of varying chain lengths (tri- to heptamers) are also known (Fig. 4). Polypyrroloindoline alkaloids are well-known constituents from the sweetshrub family (Calycanthaceae) and have received considerable attention due to their analgesic, antibacterial, antifungal, antiviral and cytotoxic activities (e.g. Canham et al. 2015; Jamison et al. 2017). Apart from the genus Eumachia, polypyrroloindoline alkaloids are also reported from a few species of Palicourea as well as Asian and Pacific species of Psychotria (see sections "Polypyrroloindoline alkaloids" under the discussion of both genera).

Oligomers are usually composed of repeating polypyrroloindoline units joined by $\mathrm{C} 3 \mathrm{a}-\mathrm{C} 7^{\prime}$ linkages interrupted by a single $\mathrm{C} 3 \mathrm{a}-\mathrm{C} 3 \mathrm{a}^{\prime}$ linkage, i.e., a chimonanthine subunit. The location (between terminal vs. internal units) of the more labile $\mathrm{C} 3 \mathrm{a}-\mathrm{C} 3 \mathrm{a}^{\prime}$ bond results in characteristic MS fragmentation patterns, and may be used to classify oligomeric polypyrroloindoline alkaloids into various subgroups. For example, in the tetrameric quadrigemine $\mathrm{B}$ the labile bond is located between unit 3 and 4 ("terminal") and it belongs to the group of $[3+1]$ polypyrroloindolines. By contrast the labile bond links units 2 and 3 ("internal") of quadrigemine $\mathrm{C}$ and the alkaloid fragments in a $[2+2]$ fashion (Jamison et al. 2017). In addition, a few compounds

Table 2 Phytochemically studied species of the genus Eumachia

\begin{tabular}{lll}
\hline Accepted species & Reported under $^{\mathrm{a}}$ & References \\
\hline Eumachia cymuligera & Eumachia cymuligera & Brand et al. (2012) \\
Eumachia depauperata & $\equiv$ Margaritopsis carrascoana & Nascimento et al. (2015a, b) \\
Eumachia forsteriana & $\equiv$ Psychotria forsteriana & Adjibadé et al. (1985, 1986, 1989, 1992), Roth et al. (1985, 1986) \\
Eumachia frutescens & $\equiv$ Hodgkinsonia frutescens & Anet et al. (1961), Fridrichsons et al. (1967, 1974), Parry et al. (1978) \\
Eumachia leptothyrsa & $=$ Psychotria beccarioides & Hart et al. (1974) \\
Eumachia lyciiflora & $\equiv$ Psychotria lyciiflora & Jannic et al. (1999) \\
Eumachia oleoides & $\equiv$ Psychotria oleoides & Guéritte-Voegelein et al. (1992), Jannic et al. (1999), Libot et al. (1987) \\
Eumachia rostrata & $\equiv$ Psychotria rostrata & Lajis et el. (1993), Mahmud et al. (1993), Takayama et al. (2004) \\
Eumachia straminea & $\equiv$ Psychotria straminea & Fu et al. (2015) \\
\hline
\end{tabular}

a Synonyms: = heterotypic synonyms, $\equiv$ homotypic synonyms 


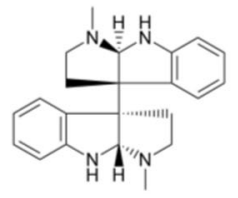

meso-chimonanthine

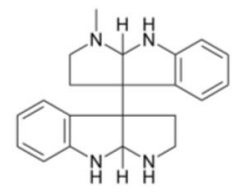

$N_{b}$-desmethyl meso-chimonanthine

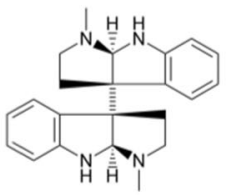

(+)-chimonanthine

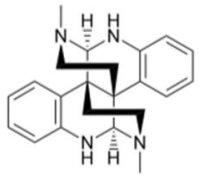

(-)-calycanthine

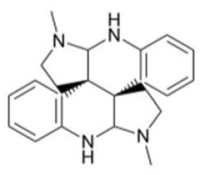

iso-calycanthine
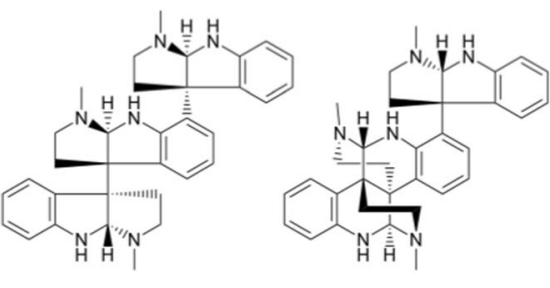

calycosidine

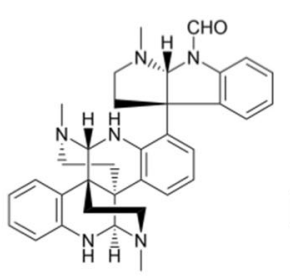

$\mathrm{N}-8$ "-formyl calycosidine

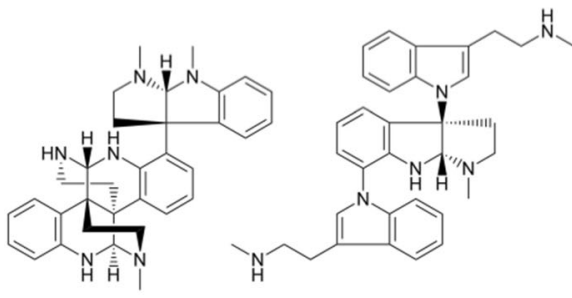

$N-8$ "-methyl- $N$-1'-demethyl

psychotrimine iso-calycosidine
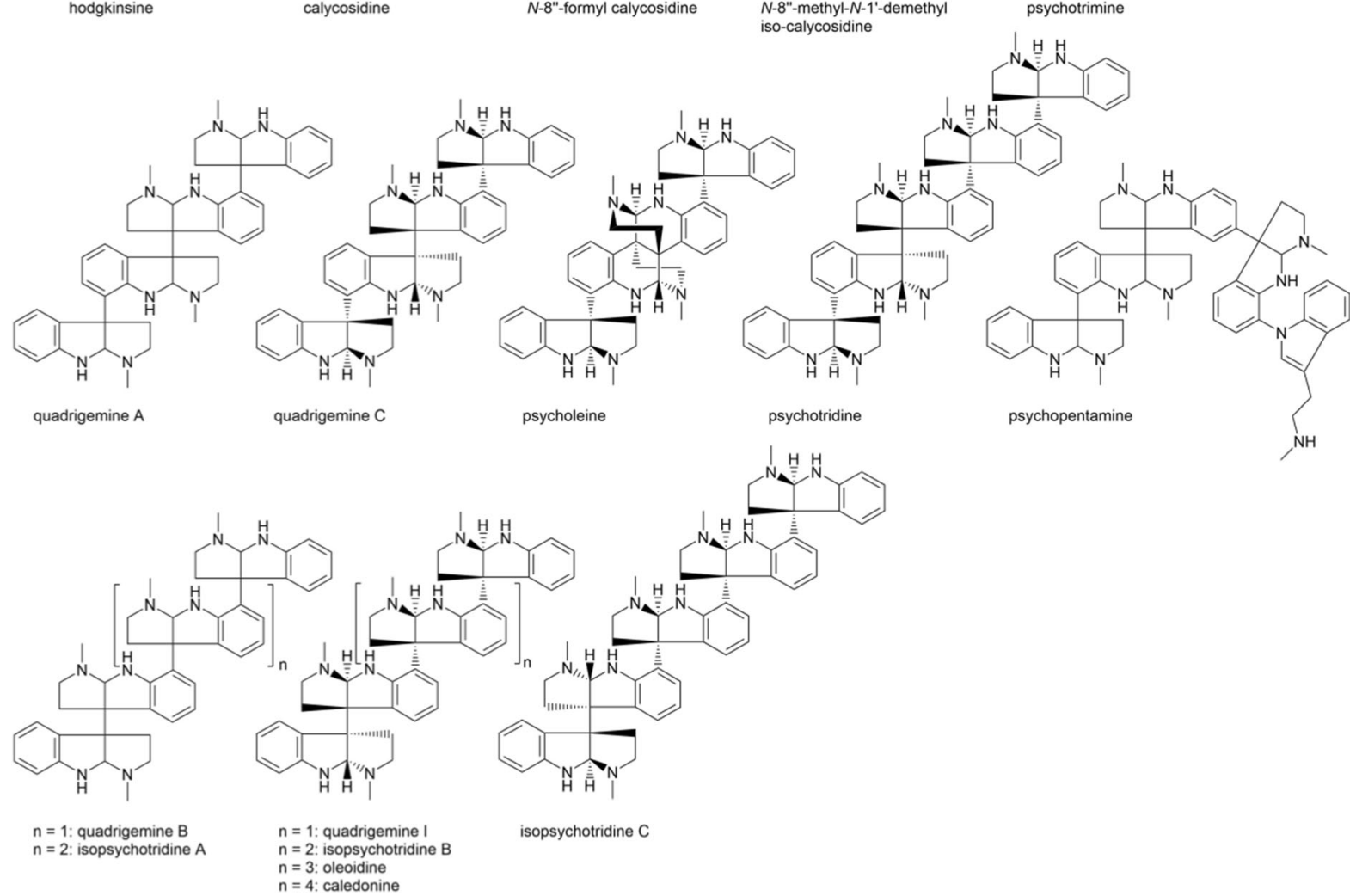

Fig. 4 Polypyrroloindoline alkaloid di- and oligomers isolated from Eumachia species. Note the unusual C-N linkage in psychotrimine and psychopentamine. Structures aligned to the central chimonanthine core. Numbering according to Jamison et al. (2017)

feature unusual $\mathrm{C}-\mathrm{N}$ linkages $\left(\mathrm{C} 3 \mathrm{a}-\mathrm{N} 1^{\prime}\right.$ or $\left.\mathrm{C} 7-\mathrm{N} 1^{\prime}\right)$ between individual units. From these, psychotrimine is unusual in having a single polypyrroloindoline unit (e.g. Takayama et al. 2004). With the exception of quadrigemine $\mathrm{C}$ whose structure was unambiguously assigned by X-ray crystallographic analysis, the exact configuration of compounds with four or more units remains to be confirmed. It was suggested that many of the named compounds are in fact identical when stereochemistry is considered (e.g. quadrigemines A, C and E; Canham et al. 2015; Jamison et al. 2017).

\section{Geophila D. Don}

Geophila is a pantropical genus with ca. 25 species, is sister to Chassalia s. str., but nested within a 
paraphyletic Chassalia s. 1. (see section "Chassalia Comm. ex Poir."). Most of its species are found in the Neotropics and Africa, with a few occurring in Asia. The genus is easily diagnosed by creeping, stoloniferous and herbaceous habit; cordate leaves with bifid stipules; white corollas; orange/red or black fruits, and often twisted pyrenes with one to several ribs that lack preformed germination slits (Razafimandimbison et al. 2014).

To date, a single species of Geophila was subject to a phytochemical investigation: Based on material collected in Yunnan Province, China, Luo et al. (2011) reported the isolation of a coumarin, a triterpene and two polyphenols from "Geophila herbacea K. Schum." However, the taxonomic identity of the studied material is problematic for a number of reasons. Geophila herbacea is a nomenclaturally superfluous and therefore illegitimate later name for Geophila repens (L.) I.M. Johnst. The latter species was long thought to be of pantropical distribution, but Razafimandimbison et al. (2014) recently showed that Geophila repens is restricted to the Neotropics. In turn the name Geophila uniflora Hiern. applies to paleotropical populations, and the name consequently applies to the species studied by Luo et al. (2011). Likewise, Rao et al. (2017) studied material of "Geophila repens" from the Chinese Guangxi province and reported the composition of its essential oil, whereas Dash et al. (2019) described the isolation of a diterpene from plants collected in the Indian state Odisha. Both accessions likewise belong to Geophila uniflora.

According to preliminary data, the Central and South American G. macropoda (Ruiz \& Pav.) DC. contains alstrostine-type alkaloids, which possess highly characteristic UV spectra (Berger, in prep.). Although data on alkaloids in the genus Geophila is scarce, the unpublished report of an alstrostine derivative is in accordance with the phylogenetic position of Geophila as sister to the alstrostine-type alkaloid containing Chassalia s. str. clade (Razafimandimbison et al. 2014).

Hymenocoleus Robbr. and Puffia Razafim. \& B. Bremer

The tropical African Hymenocoleus and the monotypic SE Malagasy endemic Puffia (both Palicoureeae) form a clade which is sister to the group of paleotropical Chassalia s. str., the 'East African Chassalia clade' and Geophila (see Fig. 1). Both genera are characterized by creeping, herbaceous and stoloniferous habit, as well as bifid stipules. They are therefore similar to species of Geophila, and have been included in that genus before Hymenocoleus and Puffia were recognized. A membranaceous sheath inside the stipules and heterostylous flowers differentiate Hymenocoleus, whereas Puffia lacks the stipular sheath and features isostylous flowers (Razafimandimbison et al. 2014; Robbrecht 1975). The phytochemistry of both genera remains unknown.

\section{Notopleura (Benth.) Bremek.}

The neotropical genus Notopleura (Palicoureeae) was long classified as Psychotria sect. Notopleura Benth. before it was finally recognized as a separate genus. As such, Notopleura is sister to Rudgea (see Fig. 1) and includes ca. 210 species distributed from Mexico and the Antilles south to Bolivia and Brazil, and it is often found in rather wet microsites or at higher elevations. The genus is generally recognized by: succulent herbaceous to subshrubby habit; stipules fused to a sheath with a single succulent glandular interpetiolarcentral appendage; terminal or more often pseudoaxillary inflorescences, small white to greenish flowers; succulent white, or black mature fruits then passing through a red stage, with 2-6, sometimes dorsiventrally flattened pyrenes with two long, ventral, marginal preformed germination slits often accompanied by a short median ventral germination slit (Razafimandimbison et al. 2014). The genus includes two subgenera: the terrestrial Notopleura subg. Notopleura and the epiphytic Notopleura subg. Viscagoga. The former subgenus includes most species and is largely diagnosed by unbranched stems, pseudoaxillary inflorescences and fruits with two pyrenes, whereas the latter includes species with branched and less succulent stems, terminal inflorescences and 2-6 pyrenes (Taylor 2001).

To date, phytochemical data on the genus Notopleura is limited to a small number of species, but all of them are devoid of alkaloids (Berger et al. 2016; Kostyan 2017). Instead, quinones were isolated from Notopleura camponutans (Dwyer \& M.V. Hayden) C.M. Taylor (Jacobs et al. 2008; Solís et al. 1995, as Psychotria camponutans (Dwyer \& M.V. Hayden) Hammel), Notopleura polyphlebia (Donn. Sm.) C.M. 
Taylor and Notopleura uliginosa (Sw.) Bremek. (Kostyan 2017). In the latter two species quinones were found together with widespread flavonoids and megastigmanes (Berger 2012; Berger et al. 2016; Kostyan 2017). Although data on additional species is urgently needed, quinones appear to characterize Notopleura.

\section{Palicourea Aubl.}

The neotropical genus Palicourea (Palicoureeae) includes at least 800 species found from the Bahamas, the Greater Antilles and Mexico south to northern Argentina. Phylogenetic studies and a re-evaluation of morphological characters have recently changed the circumscription of the genera Palicourea and Psychotria rendering both monophyletic groups (Nepokroeff et al. 1999; Razafimandimbison et al. 2014). In its 'modern' circumscription Palicourea includes Psychotria subg. Heteropsychotria and is diagnosed by: A rather greenish dried colour; persistent stipules with a sheath usually bearing two lobes or awns on each side; fruits that are metallic blue or purple-black when mature; pyrenes with preformed germination slits and seeds without an alcohol-soluble red seed coat pigment. Flower characters are notoriously variable in the genus, which is related to different pollination syndromes: Coloured inflorescence axes, large and long pedicellate flowers and vividly coloured corollas with well-developed tubes are found in hummingbirdpollinated species, and these were traditionally placed in Palicourea. By contrast, flowers of insect-pollinated species-traditionally placed in Psychotria subg. Heteropsychotria-usually have small, white, greenish, or yellow corollas with short tubes in bee-pollinated species, or white corollas with long tubes in hawk moth-pollinated species.

With phytochemical data available for 49 species, Palicourea is the best studied genus of tribes Palicoureeae and Psychotrieae. Whilst six species lack alkaloids and accumulate flavonoids, iridoids, triterpenoids and other compounds, various types of IAs characterize the remaining 43 species. If such a trend holds true for the remainder of the genus, that would easily make Palicourea the largest radiation of plants with IA formation. Accumulation of strictosidine and related MIA glucosides is reported for 36 species, and it is therefore the predominant chemical feature of the genus. Other alkaloids types include polypyrroloindoline IA in ten species, $\beta$-carbolines in seven species, simple tryptamine analogues in five species and protoalkaloids in three species. For each group of alkaloids present in Palicourea, individual structures and their source plants are briefly discussed, and biosynthetic considerations for these are found in Berger et al. (2021).

\section{"Protoalkaloids"}

Two "protoalkaloids" derived from the amino acid tyrosine were isolated from species of Palicourea: $\mathrm{N}$ Methyltyramine was found in Palicourea marcgravii A. St.-Hil. (Kemmerling 1996) and hordenine in Psychotria nemorosa Gardner (Calixto et al. 2017), for which no name is yet available in Palicourea. Furthermore, six hydroxycinnamic acid amides were detected by UPLC-MS in Palicourea sessilis (Vell.) C.M. Taylor (Samulski et al. 2020). These are derived from a condensation of hydroxycinnamic acids with biogenic amines such as putrescine being break-down products of amino acids (Macoy et al. 2015). Their structures are shown in Fig. 5.

\section{Simple indole alkaloids}

The group is composed of alkaloids derived from tryptamine without a condensation with an iridoid moiety. Thus, they are termed 'simple' IA, which stands in contrast to more complex alkaloids that are formed by the incorporation of an iridoid moiety derived from the non-mevalonate pathway. According to the mode of cyclisation and the number of monomers involved, simple IA may be divided in two subgroups, and they are discussed below. Respective biosynthetic considerations are presented by Berger et al. (2021).

Tryptamine analogues-The group includes the structurally simplest alkaloids found in the genus Palicourea: $N$-formyltryptamine from Psychotria nemorosa (Calixto et al. 2017), $N$-methyltryptamine from Palicourea hoffmannseggiana (Roem. \& Schult.) Borhidi (Naves 2014) and Palicourea sessilis (Klein-Júnior et al. 2017), $N, N, N$-trimethyltryptamine from Psychotria nuda (Cham. \& Schltdl.) Wawra (de Carvalho Junior et al. 2019) and bufotenin (5-hydroxy $N, N$-dimethyltryptamine), the hallucinogenic principle of cane toad skin (Rhinella marina (Linnaeus, 1758), Bufonidae), from Palicourea gracilenta (Müll. 
(a)<smiles>CNCCc1ccc(O)cc1</smiles>

$\mathrm{N}$-methyltyramine<smiles>CN(C)CCc1ccc(O)cc1</smiles>

hordenine (b)<smiles>NCCCCNC(=O)/C=C/c1ccc(O)cc1</smiles><smiles>COc1cc(/C=C/C(=O)NCCCCNC(=O)/C=C/c2ccc(O)c(O)c2)ccc1O</smiles>
trans- $N, N$ '-feruloyl-caffeoylputrescine<smiles>NC(N)=NCCCCNC(=O)/C=C/c1ccc(O)cc1</smiles>
trans- $N$-p-coumaroylagmatine<smiles>COc1cc(/C=C/C(=O)NCCCCN)ccc1O</smiles>

trans- $\mathrm{N}$-feruloylputrescine<smiles>COc1cc(/C=C/C(=O)NCCCCN)cc(OC)c1O</smiles>

trans- $\mathrm{N}$-sinapoylputrescine

Fig. 5 "Protoalkaloids" found in Palicourea species. (a) Simple tyrosine derivatives. (b) Hydroxycinnamic acid amides<smiles>CNCCc1c[nH]c2ccccc12</smiles>

$N$-methyltryptamine<smiles>O=CNCCc1c[nH]c2ccccc12</smiles>

N-formyltryptamine

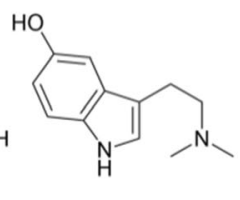

bufotenin<smiles>COc1cc(/C=C/C(=O)NCCCCN=C(N)N)ccc1O</smiles>

trans- $N$-feruloylagmatine

Fig. 6 Simple tryptamine analogues found in Palicourea species. The numbering, exemplarily shown for $N$-methyltryptamine, is taken from Ribeiro et al. (2016)

Table 3 Species of Palicourea accumulating tryptamine analogues

\begin{tabular}{lcl}
\hline Accepted species & ${\text { Reported } \text { under }^{\mathrm{a}}}^{\text {References }}$ \\
\hline Palicourea gracilenta & = Psychotria brachybotrya & Ribeiro et al. (2016) \\
Palicourea hoffmannseggiana & & Naves (2014) \\
Palicourea sessilis & & Klein-Júnior et al. (2017) \\
Palicourea comb. ined & Psychotria nuda & de Carvalho Junior et al. (2019) \\
Palicourea comb. ined & Psychotria nemorosa & Calixto et al. (2017)
\end{tabular}

a Synonymy: = heterotypic synonym; comb. ined.: combinatio inedita, nomenclatural combination under Palicourea not yet published

Arg.) Delprete \& J.H. Kirkbr. (Ribeiro et al. 2016; as Psychotria brachybotrya Müll. Arg.). Interestingly, $N$ methyltryptamine and bufotenin are related to the well-known hallucinogenic $N, N$-dimethyltryptamine (DMT), one of few alkaloids still known from the genus Psychotria in its modern circumscription (see 
Table 4 Species of Palicourea accumulating polypyrroloindoline alkaloids

\begin{tabular}{|c|c|c|}
\hline Accepted species & Reported under $^{\mathrm{a}}$ & References \\
\hline Palicourea alpina & & Woo-Ming and Stuart (1975) \\
\hline Palicourea colorata & $\equiv$ Psychotria colorata & Verotta et al. $(1998,1999)$ \\
\hline Palicourea coriacea & & da Silva et al. (2008); do Nascimento et al. (2006); Kato et al. (2017) \\
\hline Palicourea domingensis & & Ripperger (1982) \\
\hline Palicourea glomerulata & $\equiv$ Psychotria glomerulata & Solis et al. (1997) \\
\hline Palicourea hoffmannseggiana & & Naves (2014) \\
\hline Palicourea muscosa & $\equiv$ Psychotria muscosa & Jamison et al. (2017); Verotta et al. (1999) \\
\hline Palicourea ovalis & & Garcia et al. (1997) \\
\hline Palicourea semirasa & $=$ Palicourea fendleri & Nakano and Martín (1976) \\
\hline Palicourea sessilis & & Klein-Júnior et al. (2017) \\
\hline
\end{tabular}

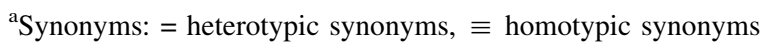

sect. "Psychotria L."). Bufotenin was isolated together with two of its dimers possessing a biphenyl core structure otherwise known only from polypyrroloindoline alkaloids (e.g. see sect. "Eumachia DC."). Brachybotryne and its $N$-oxide derivative can occur as atropisomers which is discussed in
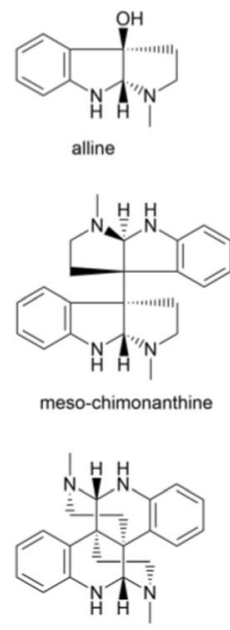

(-)-calycanthine

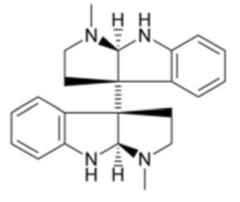

(+)-chimonanthine

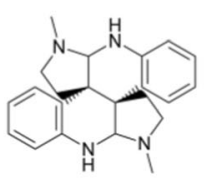

iso-calycanthine

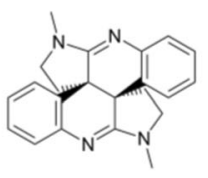

PML 100

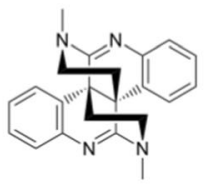

glomerulatine A

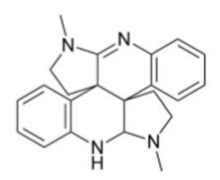

PML 300

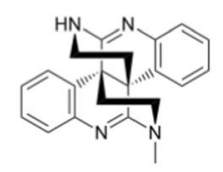

glomerulatine B

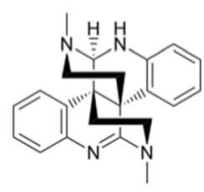

glomerulatine C

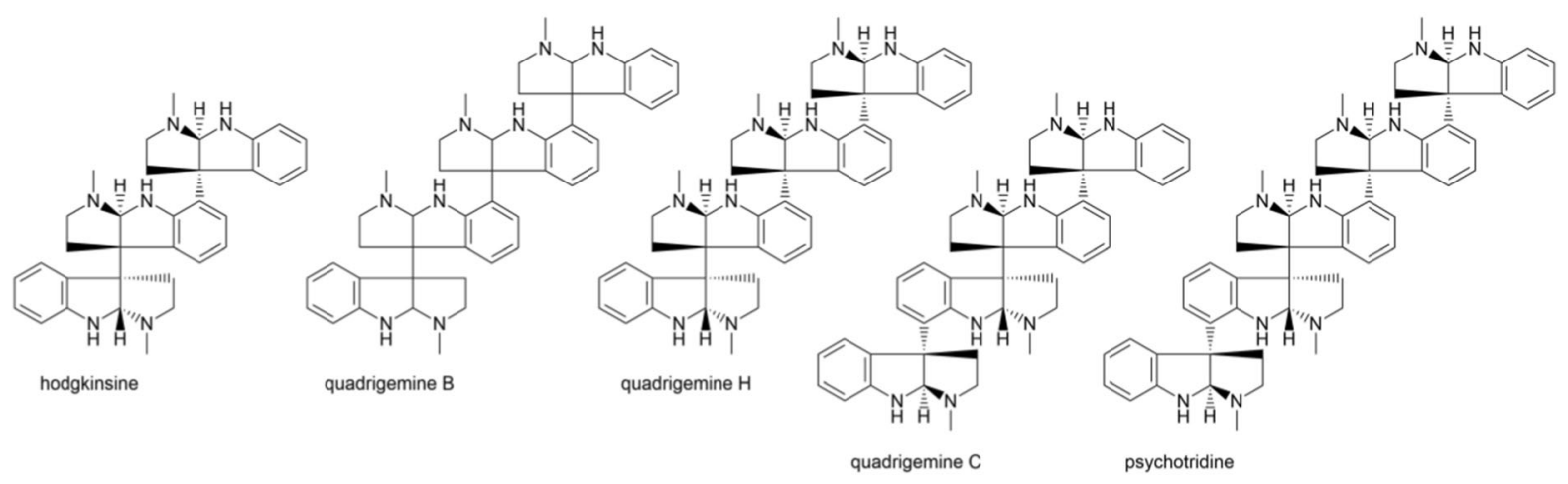

Fig. 7 Polypyrroloindoline alkaloids found in Palicourea species. Oligomers aligned to the central chimonanthine core 
detail by Ribeiro et al. (2016). However, the authors do not provide any data on optical rotation of their isolated compounds. Hence, a preferred configuration of these natural products cannot be indicated. Structures of the respective alkaloids known from Palicourea-species are shown in Fig. 6 and the species are enumerated in Table 3.

Polypyrroloindoline alkaloids-Polypyrroloindoline alkaloids, the typical chemical complement of the genus Eumachia (see sect. "Eumachia DC."), are also found in a number of species of Psychotria (see sect. "Polypyrroloindoline alkaloids") and Palicourea (see Table 4). The monomer alline was isolated from Palicourea sessilis (Klein-Júnior et al. 2017). The dimers ( +)-chimonanthine and/or (-)-calycanthine are rather widespread and occur in Palicourea alpina (Sw.) DC. (Woo-Ming and Stuart 1975), Palicourea colorata (Willd. ex Roem. \& Schult.) Delprete \& J.H. Kirkbr. (Verotta et al. 1998, 1999), Palicourea coriacea (Cham.) K. Schum. (da Silva et al. 2008; do Nascimento et al. 2006), Palicourea domingensis (Jacq.) DC. (Ripperger 1982), Palicourea glomerulata
(Donn. Sm.) Borhidi (Solis et al. 1997), Palicourea hoffmannseggiana (Naves 2014), Palicourea muscosa (Jacq.) Delprete \& J.H. Kirkbr. (Verotta et al. 1999), Palicourea ovalis Standl. (Garcia et al. 1997) and in Palicourea semirasa Standl. (Nakano and Martín 1976; as Palicourea fendleri Standl.). Other dimers are found in Palicourea glomerulata (Solis et al. 1997) and Palicourea muscosa (Verotta et al. 1999).

Oligomers are less common and are only known from Palicourea muscosa (a trimer and a tetramer; Jamison et al. 2017; Verotta et al. 1999) and Palicourea colorata (trimers to pentamers; Verotta et al. 1998, 1999). Palicourea colorata is used by the Amazonian Caboclos for its potent analgesic activity, and experimental data suggests that its alkaloids indeed affect the brain opioid system (Amador et al. 1996, 2000; Elisabetsky et al. 1995). Structures of polypyrroloindoline alkaloids isolated from Palicourea species are shown in Fig. 7.

Table 5 Species of Palicourea accumulating harmala-type $\beta$-carboline alkaloids

\begin{tabular}{lll}
\hline Accepted species & Reported under $^{\mathrm{a}}$ & References \\
\hline $\begin{array}{l}\text { Palicourea alpina } \\
\text { Palicourea deflexa }\end{array}$ & Stuart and Woo-Ming (1974) \\
Palicourea hoffmannseggiana & $=$ Psychotria barbiflora & Bertelli et al. (2017) \\
Palicourea marcgravii & E Psychotria suerrensis & de Oliveira et al. (2013); Naves (2014) \\
Palicourea suerrensis & & Kemmerling (1996) \\
Palicourea winkleri & Psychotria nemorosa & Murillo and Castro (1998) \\
Palicourea comb. ined & Berger et al. (2017) \\
\hline
\end{tabular}

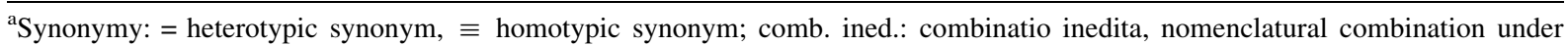
Palicourea not yet published

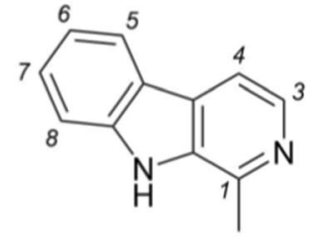

harman<smiles>Cc1nc(C(=O)O)cc2c1[nH]c1ccccc12</smiles>

harman-3-carboxylic acid<smiles>CN1CCc2c([nH]c3ccccc23)C1</smiles>

2-methyl tetrahydro$\beta$-carboline<smiles>O=C1NCCc2c1[nH]c1ccccc21</smiles>

tetrahydronorharman-1-one

Fig. 8 -Carboline type alkaloids found in Palicourea species. Numbering according to Allen and Holmstedt (1980) 
Table 6 Species of Palicourea accumulating tryptamine-secologanin type monoterpene-indole alkaloids, I. Strictosidine and related glucosides

\begin{tabular}{|c|c|c|}
\hline Accepted species & Reported under ${ }^{\mathrm{a}}$ & References \\
\hline Palicourea acuminata & & Berger et al. $(2012,2017)$ \\
\hline Palicourea adusta & & Valverde et al. (1999) \\
\hline Palicourea alpina & & do Nascimento et al. $(2006,2008)$ \\
\hline Palicourea axillaris & $\equiv$ Cephaëlis axillaris & Martín et al. (1994) \\
\hline $\begin{array}{l}\text { Palicourea } \\
\text { chiriquensis }\end{array}$ & $\equiv$ Psychotria chiriquensis & Berger (2012) \\
\hline Palicourea coriacea & & do Nascimento et al. $(2006,2008)$ \\
\hline Palicourea crocea & & Berger et al. (2015) \\
\hline Palicourea croceoides & & Berger et al. (2015) \\
\hline $\begin{array}{l}\text { Palicourea } \\
\text { cyanococca }\end{array}$ & & Berger et al. (2017) \\
\hline Palicourea deflexa & $\equiv$ Psychotria deflexa & Bertelli et al. (2015) \\
\hline Palicourea dichroa & $\equiv$ Cephaèlis dichroa & Solis et al. (1993) \\
\hline $\begin{array}{l}\text { Palicourea } \\
\text { didymocarpos }\end{array}$ & - Psychotria bahiensis & Paul et al. (2003) \\
\hline Palicourea elata & & Berger et al. (2012) \\
\hline Palicourea garciae & & Berger et al. (2017) \\
\hline $\begin{array}{l}\text { Palicourea } \\
\text { hoffmannseggiana }\end{array}$ & $=$ Psychotria barbiflora & de Oliveira et al. (2013) \\
\hline Palicourea mamillaris & $=$ Psychotria myriantha & Farias et al. (2012), Simões-Pires et al. (2006) \\
\hline Palicourea marcgravii & & Morita et al. (1989) \\
\hline Palicourea minutiflora & & Moura et al. (2020a, b) \\
\hline Palicourea padifolia & & Berger et al. (2015) \\
\hline Palicourea prunifolia & $\equiv$ Psychotria prunifolia & Faria et al. (2010), Kato et al. (2012) \\
\hline Palicourea sessilis & & Klein-Júnior et al. (2017) \\
\hline Palicourea suerrensis & & Berger et al. (2017) \\
\hline Palicourea tsakiana & & Berger et al. (2017) \\
\hline Palicourea winkleri & & Berger et al. (2017) \\
\hline \multirow[t]{2}{*}{ Palicourea comb. ined } & Psychotria laciniata & dos Santos et al. (2013a, b) \\
\hline & $\begin{array}{l}\text { Psychotria laciniata (as Psychotria } \\
\text { stenocalyx) }\end{array}$ & Queiroz et al. (2017) \\
\hline Palicourea comb. ined & Psychotria nemorosa & Calixto et al. (2017) \\
\hline Palicourea comb. ined & Psychotria nuda & de Carvalho Junior et al. (2019) \\
\hline Palicourea comb. ined & Psychotria suterella & $\begin{array}{l}\text { de Carvalho Junior et al. (2021), dos Santos et al. (2013a, b), van } \\
\text { de Santos et al. (2001) }\end{array}$ \\
\hline
\end{tabular}

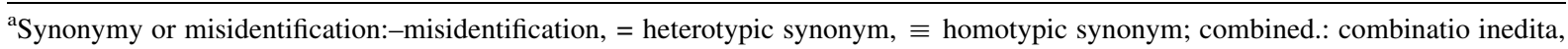
nomenclatural combination under Palicourea not yet published 

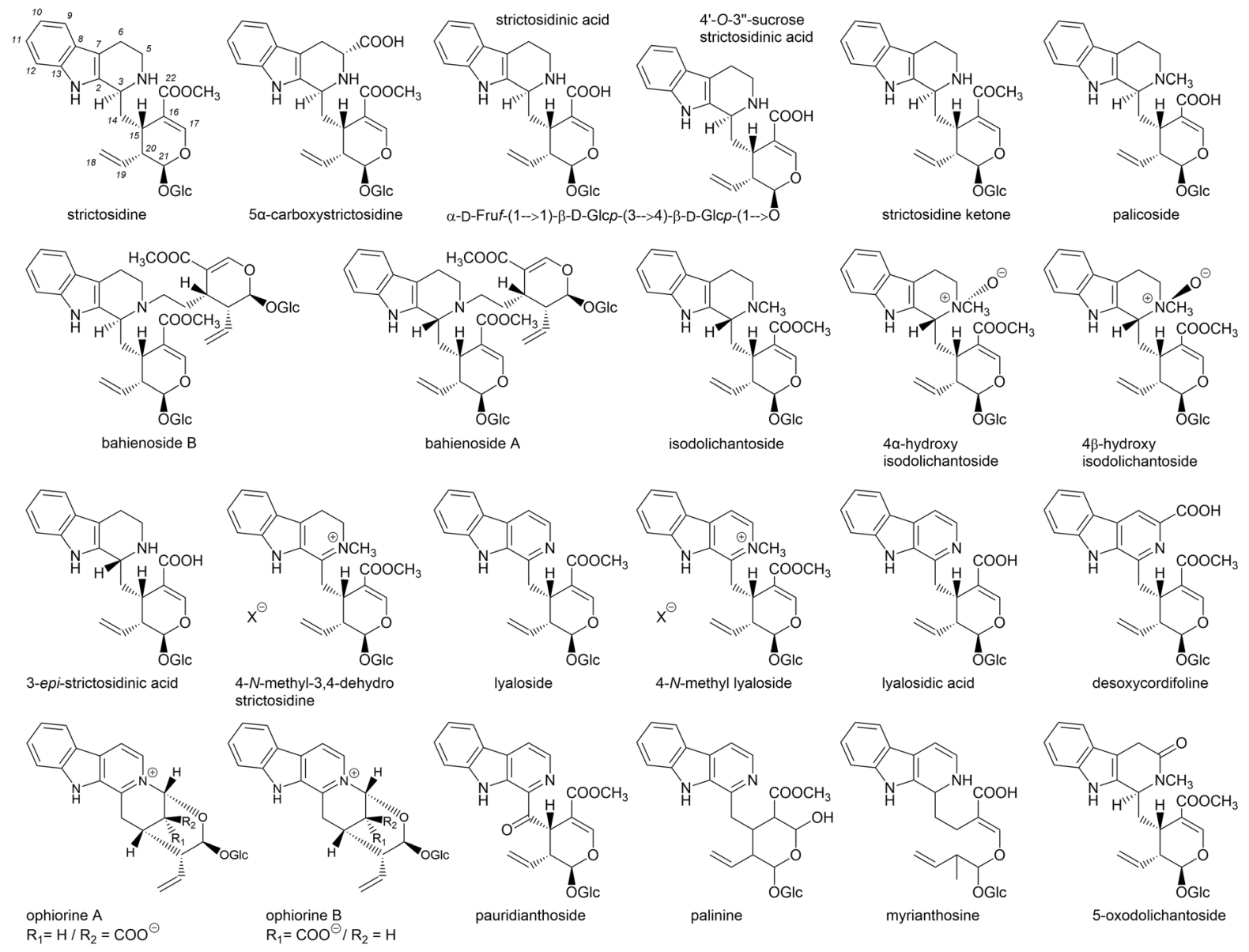

(E)-O-(6')-(4"-hydroxy-3"-methoxy)-cinnamoyl lyaloside

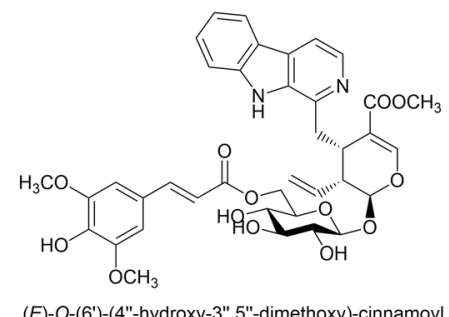

(E)-O-(6')-(4"-hydroxy-3",5"-dimethoxy)-cinnamoyl lyaloside

Fig. 9 Tryptamine-secologanin type monoterpene-indole alkaloids isolated from Palicourea species, I. Strictosidine and related glucosides. Note the additional methyl group and the unusual ring opening in myrianthosine, which distinguishes this compound from all related structures. Simões-Pires et al. (2006),

\section{$\beta$-Carbolines}

Alkaloids bearing a tricyclic pyrido(3,4- $b$ )indole skeleton are termed $\beta$-carbolines, and these show different biosynthetic origins. The present section deals with 'simple' $\beta$-carbolines that are devoid of the fused terpenoid ring system found in MIA related to who isolated the compound from Palicourea mamillaris, did not indicate a possible biosynthesis for this compound. Numbering, exemplarily shown for strictosidine, according to Silva et al. (1971)

strictosidine (see sect. "Monoterpene-indole alkaloids"). Depending on the saturation of ring $\mathrm{C}$, the group is divided in $\beta$-carboline, dihydro- $\beta$-carboline and tetrahydro- $\beta$-carboline alkaloids (Allen and Holmstedt 1980). Most simple $\beta$-carbolines are C1methylated and belong to the so-called harmala alkaloid group named after their first known source, 
Table 7 Species of Palicourea accumulating tryptamine-secologanin type monoterpene-indole alkaloids, II. Strictosamide and related glucosides featuring a pentacyclic core

\begin{tabular}{|c|c|c|}
\hline Accepted species & Reported under $^{\mathrm{a}}$ & References \\
\hline Palicourea acuminata & & Berger et al. $(2012,2017)$ \\
\hline Palicourea dichroa & $\equiv$ Cephaèlis dichroa & Solis et al. (1993) \\
\hline Palicourea mamillaris & $=$ Psychotria myriantha & Farias et al. (2012); Simões-Pires et al. (2006) \\
\hline Palicourea minutiflora & & Moura et al. (2020a, b) \\
\hline Palicourea prunifolia & $\equiv$ Psychotria prunifolia & Faria et al. (2010); Kato et al. (2012) \\
\hline $\begin{array}{l}\text { Palicourea } \\
\text { didymocarpos }\end{array}$ & - Psychotria bahiensis & Paul et al. (2003) \\
\hline Palicourea winkleri & & Berger et al. (2017) \\
\hline \multirow[t]{2}{*}{ Palicourea comb. ined } & Psychotria laciniata & dos Santos et al. (2013a, b) \\
\hline & $\begin{array}{l}\text { Psychotria laciniata (as Psychotria } \\
\text { stenocalyx) }\end{array}$ & Queiroz et al. (2017) \\
\hline Palicourea comb. ined & Psychotria leiocarpa & Henriques et al. (2004), Lopes, (1998) \\
\hline Palicourea comb. ined & Psychotria nuda & de Carvalho Junior et al. (2019); Farias et al. (2008) \\
\hline Palicourea comb. ined & Psychotria suterella & dos Santos et al. (2013a, b), van de Santos et al. (2001) \\
\hline
\end{tabular}

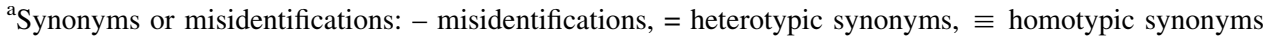

Peganum harmala L. (Nitrariaceae). Biosynthetic considerations are found in Berger et al. (2021). Harmala alkaloids act as reversible monoamine oxidase (MAO) inhibitors targeting the MAO-A isoform and are therefore of pharmacological interest in the treatment of neurodegenerative diseases (Wang et al. 2010).

Likewise, harmala alkaloid-containing species are of great ethnobotanical and ethnopharmacological importance in the preparation of ayahuasca, a traditional hallucinogenic brew used by indigenous people of the Amazon basin and adjacent areas of South America. Banisteriopsis caapi (Spruce ex Griseb.) C.V. Morton (Malpighiaceae) contains harmala alkaloids, and they provide MAO inhibition required for an oral activity of the hallucinogenic principle $\mathrm{N}, \mathrm{N}$ dimethyltryptamine (DMT) derived from the second ingredient, Psychotria viridis Ruiz \& Pav. (Callaway et al. 2005; Rivier and Lindgren 1972).

Within the genus Palicourea, harman was isolated from Palicourea alpina (Stuart and Woo-Ming 1974), Palicourea hoffmannseggiana (de Oliveira et al. 2013, as Psychotria barbiflora DC.; Naves 2014) and Palicourea suerrensis (Donn. Sm.) Borhidi (Murillo and Castro 1998). Harman-3-carboxylic acid was detected in Palicourea deflexa (DC.) Borhidi (Bertelli et al. 2017) and another derivative, tetrahydronorharman-1-one, was recently isolated from Palicourea winkleri Borhidi (Berger et al. 2017). Finally, 2-methyl tetrahydro- $\beta$-carboline (i.e. $N$-methyl

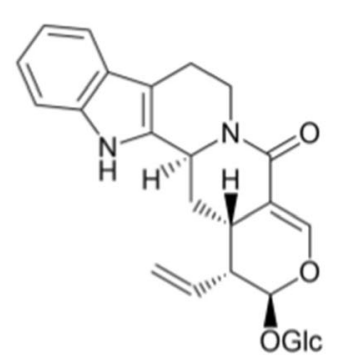

strictosamide

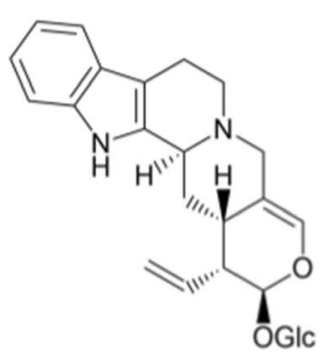

deoxostrictosamide

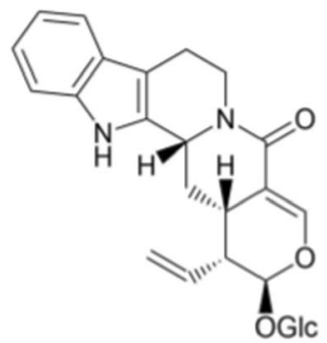

vincosamide

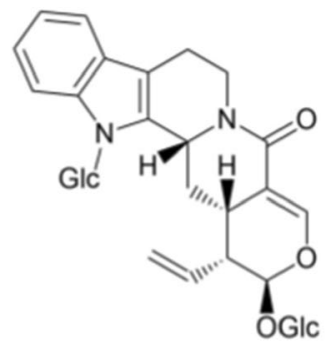

$N$, $\beta$-D-glucopyranosyl vincosamide

Fig. 10 Tryptamine-secologanin type monoterpene-indole alkaloids isolated from Palicourea species, II. Strictosamide and related pentacyclic glucosides 
(a)

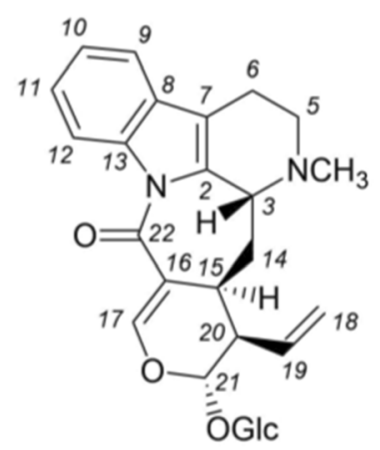

correantoside<smiles>C=C[C@@H]1[C@@H]2C[C@@H]3NC=Cc4c3n(c3ccccc43)C(=O)C2=CO[C@H]1OC(C)C</smiles>

(b)

correantosine $\mathrm{E}$<smiles>C[C@H]1OC=C2C(=O)n3c4c(c5ccccc53)CCN(C)[C@H]4C[C@H]2[C@@H]1C=O</smiles>

correantine B<smiles>C=C[C@H]1[C@H](OC(C)(C)C)OC=C2C(=O)n3c4c(c5cc(O)ccc53)CCN[C@@H]4C[C@H]21</smiles>

10-hydroxy correantoside<smiles>C=C[C@@H]1[C@@H]2Cc3nccc4c5ccccc5n(c34)C(=O)C2=CO[C@H]1OC(C)C</smiles>

correantosine $\mathrm{F}$<smiles>C[C@H]1OC=C2C(=O)n3c4c(c5ccccc53)CCN(C)[C@H]4C[C@H]2[C@H]1C=O</smiles>

20-epi-correantine B (artefact)

Fig. 11 Tryptamine-secologanin type monoterpene-indole alkaloids isolated from Palicourea species, III. Alkaloids bearing an azepane moiety. (a) Glucosides: Correantosides. Stachyoside is notable because it lacks one carbon atom in the basic structure and it cannot be ruled out that the biosynthesis is not directly related to the other compounds in this series.

1,2,3,4-tetrahydro- $\beta$-carboline), an alkaloid that lacks the C1-methylation distinctive for harmala alkaloids was isolated from Palicourea hoffmannseggiana (Naves 2014), Palicourea marcgravii (Kemmerling 1996) and Psychotria nemorosa (Calixto et al. 2017).<smiles>C=C[C@H]1[C@H](OC(C)=O)OC=C2C(=O)n3c4c(c5ccccc53)CCN(C=O)[C@H]4C[C@H]21</smiles>

nor-methyl-23-oxo correantoside

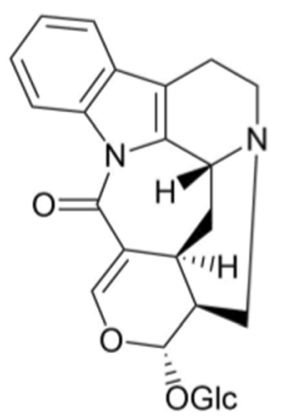

stachyoside<smiles></smiles>

correantine C<smiles>C=C[C@@H]1[C@@H]2C[C@@H]3NCCc4c3n(c3ccccc43)C(=O)C2=CO[C@H]1OC(C)C</smiles>

$\mathrm{N}$-demethyl correantoside<smiles>COC(=O)C1=CO[C@@H](C)[C@@H]2[C@@H]1C[C@@H]1NCCc3c1n(c1ccccc31)[C@@H]2O</smiles>

correantine A
However, due to the presence of the azepane moiety, stachyoside is assigned here and it is drawn based on the illustration of the other structures. (b) Aglycones: Correantines. Numbering, exemplarily shown for correantoside, according to Achenbach et al. (1995)

Species of Palicourea accumulating $\beta$-carboline alkaloids are enumerated in Table 5 and the respective structures are shown in Fig. 8. 
Table 8 Species of Palicourea accumulating tryptamine-secologanin type monoterpene-indole alkaloids, III. Strictosidine- and strictosamide-derived aglycones

\begin{tabular}{lll}
\hline Accepted species & Reported under $^{\mathrm{a}}$ & References \\
\hline $\begin{array}{l}\text { Palicourea acuminata } \\
\text { Palicourea axillaris }\end{array}$ & E Cephaèlis axillaris & Berger et al. (2012) \\
Palicourea cyanococca & Martín et al. (1994) \\
Palicourea dichroa & E Cephaélis dichroa & Berger et al. (2017) \\
Palicourea prunifolia & E Psychotria prunifolia & Solis et al. (1993) \\
Palicourea rigida & & Faria et al. (2010), Kato et al. (2012) \\
Palicourea didymocarpos & - Psychotria bahiensis & Vencato et al. (2006) \\
Palicourea comb. ined & Psychotria laciniata & Paul et al. (2003) \\
Palicourea comb. ined & Psychotria suterella & dos Santos et al. (2013a, b) \\
\hline
\end{tabular}

${ }^{\text {a }}$ Synonyms or misidentifications:- misidentifications, $\equiv$ homotypic synonyms<smiles>C=CCC1=CN2CCc3c(n(CCl)c4ccccc34)[C@H]2C[C@H]1[C@@H](C=C)C(=O)OC</smiles>

lagamboside<smiles>C/C=C\C=C(/C=O)[C@]1(C)C[C@H]2c3[nH]c4ccccc4c3CCN2C=C1C(=O)OC</smiles>

E-vallesiachotamine<smiles>C/C=C\C(=O)C1=CN2CCc3c([nH]c4ccccc34)[C@H]2C[C@H]1/C(C=O)=C/C</smiles>

Z-vallesiachotamine<smiles>COC(=O)C1=CN2CCc3c([nH]c4ccccc34)[C@H]2C[C@H]1C1=CCOC1=O</smiles>

vallesiachotamine lactone<smiles>C=C[C@H](CO)[C@H]1CCN2CCc3c([nH]c4ccc(O)cc34)[C@H]2C1</smiles>

10-hydroxy antirhine<smiles>C=Cc1cncc2c(=O)n3c(cc12)-c1[nH]c2ccccc2c1CC3</smiles>

angustine<smiles>C=C[C@H](CO)[C@H]1CC[N+]2([O-])CCc3c([nH]c4ccc(O)cc34)[C@H]2C1</smiles>

10-hydroxy antirhine $\mathrm{N}$-oxide<smiles>CC(=O)c1cncc2c(=O)n3c(cc12)-c1[nH]c2ccccc2c1CC3</smiles>

naucletine<smiles>C=C[C@H](CO)[C@H](CCO)Cc1nccc2c1[nH]c1ccc(O)cc12</smiles>

10-hydroxy isodeppeaninol

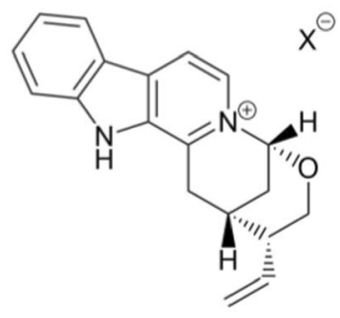

prunifoleine

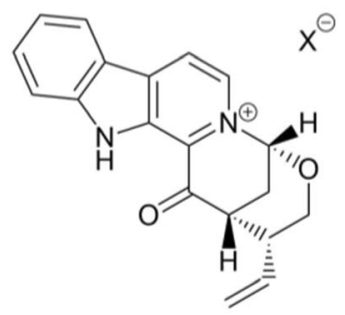

14-oxoprunifoleine

Fig. 12 Tryptamine-secologanin type monoterpene-indole alkaloids isolated from Palicourea species, IV. Strictosidine- and strictosamide-derived aglycones. Numbering, exemplarily shown for lagamboside, according to Berger et al. (2012) 
(a)

$$
\text { (2) }
$$

javaniside

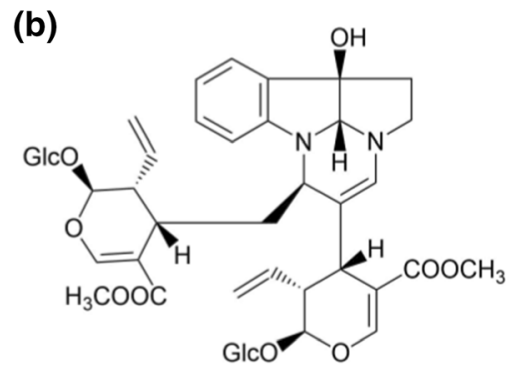

isoalstrostine $\mathrm{A}$

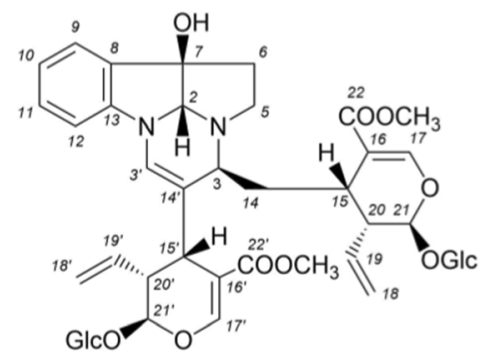

alstrostine $\mathrm{A}$

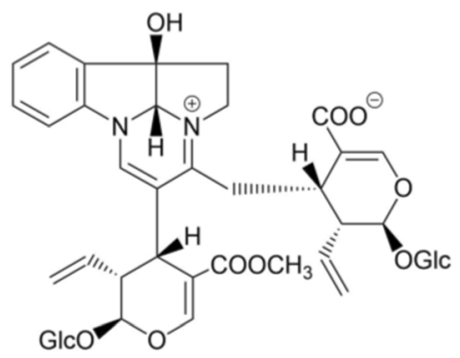

dehydrorudgeifoline
Fig. 13 Tryptamine-secologanin type monoterpene-indole alkaloids isolated from Palicourea species, V: Alkaloids from Palicourea luxurians (Rusby) Borhidi. (a) Javaniside, a spirocyclic oxindole alkaloid; (b) Alstrostine-type alkaloids.
Alstrostine A was also isolated from Chassalia curviflora (Wall.) Thwaites, see sect. "Chassalia Comm. ex Poir.". Numbering of javaniside according to Ma \& Hecht (2004), numbering of alstrostine A is according to Cai et al. (2011)

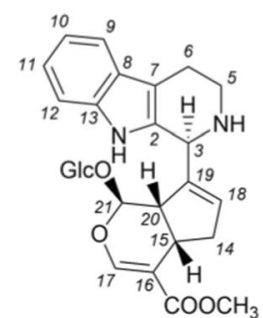

psychollatine

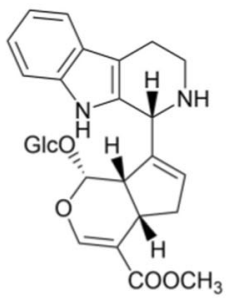

croceaine A

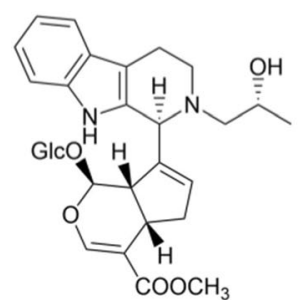

$N^{4}$-(R)-2-hydroxypropyl psychollatine

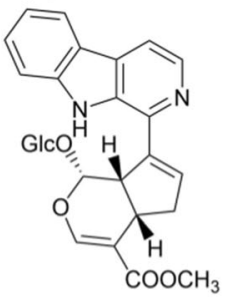

croceaine B

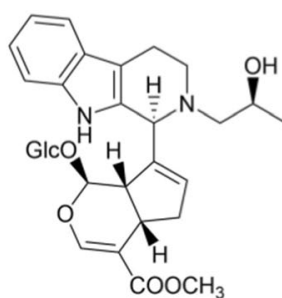

$N^{4}$-(S)-2-hydroxypropyl psychollatine

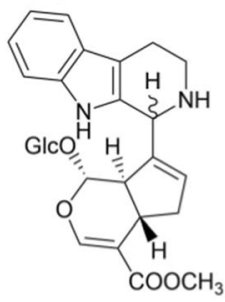

umbellatine

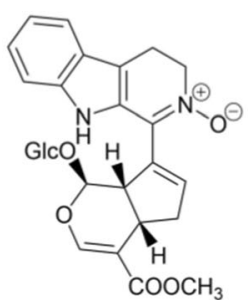

3,4-dehydropsychollatine $N^{2}$-oxide

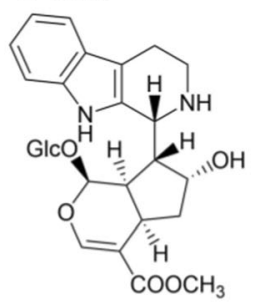

brachycerine

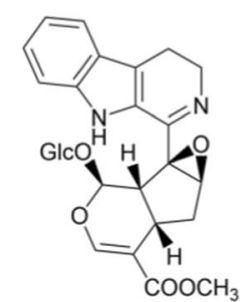

3,4-dehydro-18,19- $\beta$-epoxy psychollatine

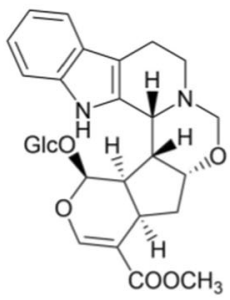

palicroceaine

Fig. 14 Tryptamine-loganin type monoterpene-indole alkaloids isolated from Palicourea species. Numbering according to Kerber et al. (2001), but other numbering schemes are also used (Berger et al. 2015, see supplementary figure S1)

Monoterpene-indole alkaloids

The basic biosynthetic steps towards MIA are well known and the key role of strictosidine synthase has already been addressed above. This enzyme catalyses the stereospecific PSR between the amine function of tryptamine and the aldehyde function of secologanin. The resulting tetrahydro- $\beta$-carboline core represents 
Table 9 Species of Palicourea accumulating tryptamine-loganin type monoterpene-indole alkaloids

\begin{tabular}{lll}
\hline Accepted species & Reported under $^{\mathrm{a}}$ & References \\
\hline Palicourea brachypoda & $=$ Psychotria umbellata & Both et al. (2002), Kerber et al. (2008, 2014) \\
Palicourea crocea & & Berger et al. (2015), Düsman et al. (2004), Narine and Maxwell (2009) \\
$\begin{array}{l}\text { Palicourea fastigiata } \\
\text { Palicourea } \text { comb. ined }\end{array}$ & Berger et al. (2015) \\
\hline
\end{tabular}

asynonyms: = heterotypic synonyms

the basic structure of all tryptamine-iridoid alkaloids. Among these, compounds possessing a secologanin moiety ("tryptamine-secologanin alkaloids") and compounds with a loganin moiety ("tryptamineloganin alkaloids") may be differentiated (see below). The cores of tryptamine-iridoid alkaloids may be subjected to various modifications, and corresponding biosynthetic considerations are found in Berger et al. (2021).

\section{Tryptamine-secologanin type MIA}

\section{Strictosidine and related glucosides}

Accumulation of strictosidine and 23 related glucosides is reported from 28 species of Palicourea (see Table 6). Interestingly, most of these strictosidinederived alkaloids retain the glucose moiety, which is remarkable because a certain level of chemical diversity is created even by omitting the deglucosylation step, otherwise considered the gateway to MIA diversity (Barleben et al. 2007; O'Connor and Maresh 2006). In many species, derivatives with tetrahydro- $\beta$ carboline (e.g. strictosidine) and $\beta$-carboline cores (e.g. lyaloside) co-occur.

Most alkaloids show only minor modifications leaving the basic strictosidine skeleton unchanged.

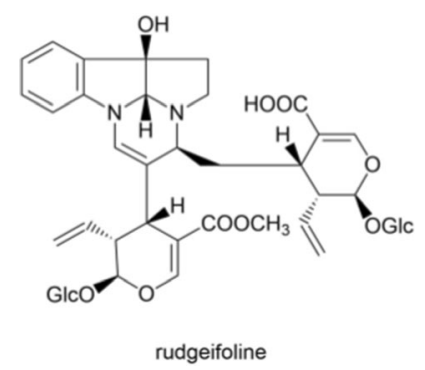

Fig. 15 Alkaloids isolated from Rudgea species. Rudgeifoline from Rudgea cornifolia (Kunth) Standl.
Corresponding structures are shown in Fig. 9. However, some species (appendix, Table 11) accumulate alkaloid glucosides with structural modifications such as ring cleavage or additional ring formations. Examples include ophiorines A and B, first reported from the genus Ophiorrhiza (Aimi et al. 1985). These compounds possess a unique $\mathrm{N}-4-\mathrm{C}-17$ linkage creating an additional heterocycle, but they retain their glucose moiety and the carboxyl group from the iridoid function. According to their (positively charged) quaternary ammonium cation and negatively charged carboxyl group, these are classified as betaine type tryptamine-iridoid alkaloids. Within Palicourea, ophiorines are only known from Palicourea suerrensis (Berger et al. 2017).

\section{Strictosamide and related glucosides}

Strictosamide features a pentacyclic core and a lactam ring resulting from a condensation of the secondary amine and the carboxyl group derived from secologanin (see Berger et al. 2021). Strictosamide is found in 11 species of Palicourea (see Table 7) such as in Palicourea winkleri, where it occurs together with the recently described deoxostrictosamide (Berger et al. 2017). By contrast the stereoisomer vincosamide appears to be of restricted occurrence and was isolated even more recently from Palicourea minutiflora (Müll. Arg.) C.M. Taylor (Moura et al. 2020a, b). In addition the related $N$ - $\beta$-D-glucopyranosyl vincosamide was reported from Psychotria leiocarpa Cham. \& Schltdl. (Henriques et al. 2004). The respective structures are shown in Fig. 10.

\section{Correantosides and correantines}

Correantosides and the related correantines are a group of unusual MIA featuring an azepane moiety, which is derived by an intramolecular cyclization 
Table 10 Alkaloid-accumulating species of Psychotria (Psychotrieae). All species except Psychotria viridis contain polypyrroloindoline alkaloids

\begin{tabular}{|c|c|c|}
\hline Accepted species & Reported under ${ }^{\mathrm{a}}$ & References \\
\hline Psychotria calocarpa & & Zhou et al. (2010) \\
\hline Psychotria henryi & & Liu et al. $(2013,2014)$ \\
\hline Psychotria malayana & & Hadi and Bremner (2001), Hadi et al. (2014) \\
\hline Psychotria milnei & $\equiv$ Calycodendron milnei & Adjibadé et al. (1990), Libot et al. (1987, 1988), Saad et al. (1995) \\
\hline Psychotria pilifera & & Li et al. (2011b), Liu et al. (2016) \\
\hline Psychotria viridis & & Callaway et al. (2005), Rivier and Lindgren (1972), Soares et al. (2017) \\
\hline
\end{tabular}

${ }^{a}$ Synonyms: $\equiv$ homotypic synonym

between $N-1$ and the iridoid framework. Whilst correantosides are glucosides and retain the exocyclic ethylene group from secologanin, correantines are aglycones showing a different mode of ring formation and resulting positions of functional groups. Berger et al. (2021) postulated a probable biosynthesis. Correantines and correantosides appear to be of restricted distribution within Palicourea, they are only known from Palicourea correae (Dwyer \& M.V. Hayden) Borhidi (Achenbach et al. 1995) and Psychotria stachyoides Benth. (Pimenta et al. 2010a, b, 2011). The respective structures are shown in Fig. 11.

\section{Strictosidine- and strictosamide-derived aglycones}

Aglycones of strictosidine and strictosamide are infrequently encountered in Palicourea, and appear to be restricted to a few species (Table 8), in which they are usually accompanied by related glucosides (appendix, Table 11). The cleavage of the glucose moiety by a dedicated strictosidine $\beta$-glucosidase (SGD; Barleben et al. 2007) leads to a spontaneous ring opening and creates a reactive dialdehyde intermediate, which ultimately converts to modified carbon skeletons with open sidechains or new ring formations. Berger et al. (2021) postulated a probable biosynthesis. Some of these aglycones show complex structural features and many are of great pharmacological importance (O'Connor and Maresh 2006). Within the genus Palicourea, comparably simple structures of strictosidine-derived alkaloid aglycones are found and these are shown in Fig. 12.

\section{Javaniside}

Javaniside was recently isolated from Palicourea luxurians (Rusby) Borhidi, and represents the only spirocyclic oxindole alkaloid so far reported from the genus Palicourea (Kornpointner et al. 2020). Alkaloids with a spiro structure i.e. cycles fused at a central carbon, are well-known from species of the genus Uncaria (Rubiaceae) and probably contribute to its bioactivity (e.g. Muhammad et al. 2001; Wang et al. 2011). The structure of javaniside is shown in Fig. 13a and a biosynthetic scheme is found in Berger et al. (2021).

\section{Alstrostines}

Within Rubiaceae, alstrostine-type MIA were previously isolated from a single species of Chassalia and Rudgea (Schinnerl et al. 2012), and they are discussed in section "Chassalia Comm. ex Poir.". Alstrostine A, dehydro-rudgeifoline and iso-alstrostine A were rather recently reported also from Palicourea luxurians (Kornpointner et al. 2020). That record represents the first and only occurrence in the large genus Palicourea (Fig. 13b).

\section{Tryptamine-loganin type MIA}

Contrary to the above-mentioned secologanin-derived MIA, a structurally related group features a loganin instead of a secologanin moiety. Structures of the respective alkaloids known from Palicourea species are shown in Fig. 14 and listed in Table 9. So far, these alkaloids have been reported from four species, Palicourea brachypoda (Müll. Arg.) L.B. Sm. \& Downs (Both et al. 2002, as Psychotria umbellata 
Vell.; Kerber et al. 2008, 2014, as Psychotria umbellata Thonn.), Palicourea crocea (Sw.) Roem. \& Schult. (Berger et al. 2015; Düsman et al. 2004; Narine and Maxwell 2009), Palicourea fastigiata Kunth (Berger et al. 2015) and Psychotria brachyceras Müll. Arg. (Kerber et al. 2001). Based on morphology the species have been classified in three different sections in the last complete monograph of the Brazilian Psychotria alliance (Müller Argoviensis 1881) and are also considered unrelated here (Berger, pers. obs.). Although phylogenetic data is necessary to clarify their relationships, this indicates that the change from tryptamine-secologanin to tryptamineloganin alkaloids could have occurred several times. Loganin and secologanin are biosynthetically related and Berger et al. (2021) proposed a biosynthetic scheme for tryptamine-loganin alkaloids.

\section{Rudgea Salisb.}

The neotropical genus Rudgea (Palicoureeae) includes more than 150 species of shrubs and small to occasionally larger trees found from Mexico and the Lesser Antilles south to northern Argentina. The circumscription of the genus has always been rather stable and unproblematic when compared to that of other lineages of the tribe: Rudgea is diagnosed by persistent or fragmenting, entire, round, truncate to acute stipules with marginal glands or medial groups of glandular appendages, which are usually early caducous; terminal, and often whitish inflorescences, bright white, small to rather large, fragrant corollas, some of which possess conspicuous appendages on the lobes; comparably large, white, orange/red or black spongy to fleshy drupes, and dorsally smooth to ridged planoconvex, and ventrally flat but deeply furrowed pyrenes with 2 marginal and 1-3 abaxial preformed germination slits. Although Rudgea and Notopleura are very different morphologically, they show a wellsupported sister-group relationship (Bruniera 2015; Razafimandimbison et al. 2014; Zappi 2003; see Fig. 1).

To date, three species of Rudgea have been phytochemically studied. Alkaloids were found only in Rudgea cornifolia (Kunth) Standl. which yielded rudgeifoline, an alstrostine-type alkaloid (Fig. 15; Schinnerl et al. 2012). Similar alkaloids are also known from single species of Chassalia, Geophila and Palicourea (see above). The other two species deviate by accumulating triterpenes and quinones (de Cacia et al. 2007; Lopes et al. 1999; Young et al. 1998).

\section{Psychotrieae}

\section{Psychotria L.}

Psychotria is a pantropical genus that includes at least 1,600 species and is among the largest genera of flowering plants. The genus comprises of seven lineages with different distribution ranges including the 'Afro-Asian-WIOR-neotropical Psychotria clade', the 'Afro-neotropical Psychotria clade' or the 'Pacific Psychotria clade'. Psychotria is paraphyletic in respect to the myrmecophytic Hydnophytinae nested within the latter subgroup. The recent transfer of most species of Psychotria subg. Heteropsychotria to Palicourea renders Psychotria a monophyletic group if the Hydnophytinae are formally included in Psychotria, as suggested by Razafimandimbison et al. (2014). In its current circumscription Psychotria is largely diagnosed by the following characters: A reddish-brown, grayish to blackish dried colour; interpetiolar, triangular and caducous stipules leaving a stipular scar with ferruginous hairs when shed; flowers adapted to insect pollination and characterized by small size, straight tubes and white, cream or greenish corollas; red or rarely white drupaceous fruits; seeds with an alcohol-soluble red seed coat pigment and pyrenes without preformed germination slits. However, numerous exceptions such as different fruit or flower colours occur in part of the range of the genus (e.g. Lachenaud 2019; Taylor 1996, 2020; Taylor et al. 2020).

Without taking two decades of taxonomic progress in the generic classification of Palicoureeae and Psychotrieae into account (see sections "Taxonomy of Palicoureeae and Psychotrieae" and "Palicourea Aubl."), recent phytochemical reviews have regarded various classes of IA and MIA as characterising the genus Psychotria (Calixto et al. 2016; de Carvalho Junior et al. 2017; Martins and Nunez 2015; Yang et al. 2016). The here-presented dataset applies an updated generic classification and challenges the previous assumption of Psychotria as an alkaloid-rich genus. Furthermore, it calls for a revised chemosystematic view based upon the currently accepted taxonomic concepts. The present review highlights 
that all reports of MIA and most reports of other alkaloid groups from Psychotria pertain to species now assigned to Carapichea, Eumachia and Palicourea, leaving only few species with alkaloids (see Table 10): A single species $(2.1 \%$ of all studied Psychotria) contains simple tryptamine analogues and five species (10.6\%) accumulate polypyrroloindoline alkaloids, whereas the remaining 41 studied species (87.2\%) are devoid of alkaloids (Table 10; appendix Table 11).

Published phytochemical data is currently available for 47 species of Psychotria corresponding to only $2.9 \%$ of its known diversity. Hence, the state of phytochemical research is extremely limited, and even more, these studies are unevenly distributed over the range of the genus. For example, only four species of the Continental African flora with ca. 240+ species (Lachenaud 2019) were studied and all of them are devoid of alkaloids: tannins are reported from Psychotria brandneriana (L. Linden) Robbr., Psychotria capensis Vatke and Psychotria orophila E.M.A. Petit (Berger 2012). Additionally, Psychotria capensis yielded $\beta$-sitosterol and an unidentified carotenoid derivative (Kafua et al. 2009), and a number of polyamines, polyphenols and other compounds were identified in Psychotria punctata Vatke by UPLC-MS. One of these is pavettamine that causes gousiektedisease in livestock (Schindler et al. 2021; Van Elst et al. 2013, as Psychotria kirkii Hiern; see Lachenaud 2019). Furthermore, the $\mathrm{C}_{7} \mathrm{~N}$ aminocyclitol kirmamine was isolated from bacterial nodules of Psychotria punctata and it was proposed that the compound is formed by its obligate leaf symbiont “Candidatus Caballeronia kirkii" (Sieber et al. 2015, as Psychotria kirkii and "Candidatus Burkholderia kirkii"). Furthermore, none of the species of the very rich Malagasy flora with $150+$ endemic species was studied (Taylor 2020; Taylor et al. 2020).

The 200+ species of Neotropical Psychotria are resolved in two clades (Razafimandimbison et al. 2014), but only three species from the group have received some initial study. Apigenin 7-O- $\alpha-\mathrm{L}-$ rhamnopyranosyl-( $1 \rightarrow 6)-\beta$-D-glucopyranoside was isolated from Psychotria nervosa (Berger et al. 2016), the common triterpenoids $\beta$-sitosterol and ursolic acid were found in Psychotria carthagenensis Jacq. (Leal and Elisabetsky 1996; Lopes et al. 2000) and the well-known hallucinogenic principle $N, N$ dimethyltryptamine (DMT) and related compounds (see below) were found in the ethnobotanically important Psychotria viridis. Preliminary HPLCUV/VIS analyses of 17 species from the morphologically and phylogenetically diverse Costa Rican flora (e.g. Berger and Schinnerl 2019; Taylor 2014) consistently showed a lack of alkaloids. Instead, accumulation of condensed tannins prevails which is also supported by an exceptionally high total phenolic content ranging from 277-454 mg gallic acid equivalents (GAE)/g of dry extract measured by the FolinCiocalteu reagent method (Berger 2012; Berger et al., unpublished data). Together with data from other regions (appendix, Table 11), this suggests that condensed tannins characterize the genus, which renders Psychotria the largest genus characterized by the accumulation of tannins. Furthermore, traces of asperuloside were detected in developing leaves of a few species (Berger 2012; Berger et al., unpublished data).

Simple indole alkaloids

Tryptamine analogues-The ethnobotanically important and well-studied Psychotria viridis is the only species of the genus known to contain simple tryptamine analogues. It yields the well-known hallucinogenic principle $N, N$-dimethyltryptamine (DMT) and the related $N$-methyltryptamine together with the $\beta$-carboline alkaloid 2-methyl tetrahydro- $\beta$-carboline (e.g. Callaway et al. 2005; Rivier and Lindgren 1972; Soares et al. 2017; see also the corresponding compound classes under sect. "Palicourea Aubl."). Reports on DMT content in other species such as Psychotria carthagenensis have so far proven erroneous and may have been based on misidentification of surveyed plants (Leal and Elisabetsky 1996; Lopes et al. 2000).

Polypyrroloindoline alkaloids-About half of the species of Psychotria subjected to phytochemical investigation occur in the Asian and Pacific region. 19 of these are devoid of alkaloids, instead accumulating various iridoids, polyphenols, terpenoids and other groups of specialized metabolites (see appendix, Table 11). Polypyrroloindoline alkaloids were reported from the remaining five species (Table 10), and first isolated from Psychotria milnei (A. Gray) K. Schum. (Adjibadé et al. 1990; Libot et al. 1987, 1988; Saad et al. 1995; as Calycodendron milnei (A. Gray) A.C. Sm.). Based on an expanded calyx, the species 
endemic to Fiji and Vanuatu was initially placed in the genera Calycosia and Calycodendron, but later transferred to Psychotria. DNA phylogenetic data has shown that it belongs to the Pacific clade which includes some of the more derived or morphologically aberrant species such as the epiphytic tuberous myrmecophytic Hydnophytinae (Barrabé et al. 2014) (Fig. 16). Other species with similar alkaloids include Psychotria calocarpa Kurz (Zhou et al. 2010), Psychotria henryi H. Lév. (Liu et al. 2013, 2014; some with unusual $\mathrm{C} 3 \mathrm{a}^{\prime}-\mathrm{N} 1$ linkage), Psychotria malayana Jack. (Hadi and Bremner 2001; Hadi et al. 2014; but the species has sometimes been confused with species of Eumachia, see Taylor et al. 2017: 316) and Psychotria pilifera Hutch. (Li et al. 2011b). All polypyrroloindoline alkaloids reported from the genus Psychotria are shown in Fig. 17 (see Sects. "Eumachia DC." and "Palicourea Aubl." for genera of the Palicoureeae accumulating these IA).

\section{$\beta$-Carbolines}

Two $\beta$-carboline alkaloids were reported from the genus Psychotria, see sect. " $\beta$-Carbolines" under the genus Palicourea for some information on that class of alkaloids. GC-MS indicated the presence of 2-methyl tetrahydro- $\beta$-carboline in Psychotria viridis (Rivier and Lindgren 1972) and 3-methyl tetrahydro$\gamma$-carboline in Psychotria malayana (Hadi et al. 2014). The structure of the latter was determined from GCMS data and has not further been proven. It is unlikely that this structure resulted directly from a PictetSpengler reaction. It is probably a rearrangement product of chimonanthine or calycanthine, which was also described by the same authors from $P$. malayana. Finally, 2-methyl tetrahydro- $\beta$-carboline was also isolated from Psychotria pilifera (Liu et al. 2016, but

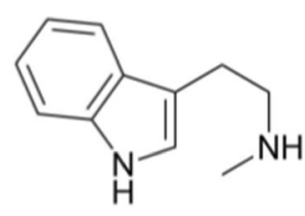

$\mathrm{N}$-methyltryptamine

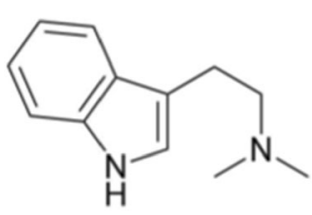

$\mathrm{N}, \mathrm{N}$-dimethyltryptamine
Fig. 16 Alkaloids isolated from Psychotria species, I. Tryptamine analogues see below) and corresponding structures are shown in Fig. 18.

Monoterpene-indole alkaloids from Psychotria pilifera?

To date a single study has reported the isolation of MIA from the genus Psychotria. Together with four polypyrroloindoline IA, Liu et al. (2016) described the occurrence of MIA with highly derived skeletons from Psychotria pilifera collected in Yunnan Province, China (see supplementary Fig. S2). The occurrence of such alkaloids largely confined to the family Apocynaceae is unparalleled in genus, as well as in the entire Psychotrieae and Palicoureeae rendering the taxonomic entity of the studied plant material doubtful. Instead the reported chemical complement fits to a number of Apocynaceae, which could be confused with Rubiaceae, especially when sterile specimens or bulk material are collected for isolation. For example, most structural groups and even individual MIA reported for Psychotria pilifera have been isolated from Tabernaemontana cymosa Jacq. and are also present in other species of the genus (Achenbach et al. 1997). Hence, an adulteration of material of Psychotria pilifera with a species of Apocynaceae is suggested, and this explanation appears probable given the large amount ( $8 \mathrm{~kg}$ dry mass) collected from this rather rare and slender understory shrub (Chen and Taylor 2011). A second phytochemical study on Psychotria pilifera (Li et al. 2011b) reported the occurrence of only polypyrroloindoline IA, likewise supporting such an assumption. Hence, these compounds are therefore tentatively excluded from the genus Psychotria pending further study.

Curiously, the same species also afforded $\mathrm{N}$ methylcarbazole (i.e. 9-methylcarbazole) (see supplementary Fig. S3; Liu et al. 2016), a tricyclic carbazole and a potent procarcinogenic component of tobacco smoke particulate matter, diesel fuel, domestic and industrial wastewater, and other sources of pollutant emissions (e.g. da Cunha et al. 2016). Carbazoles are characterized by an indole moiety annulated with a benzene ring, but originate from the anthranilic acid pathway via a 3-prenylquinolon and a 2-prenylindole to 3-methylcarbazole. They are therefore not derived from the amino acid tryptophan as suggested by the indole moiety. Within plants naturally occurring carbazoles are largely restricted to the Rutaceae family 


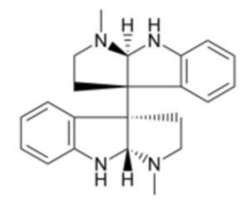

meso-chimonanthine<smiles>CNCCc1cn([C@H]2CCN(C)[C@H]2Nc2ccccc2)c2ccccc12</smiles>

psychotriasine

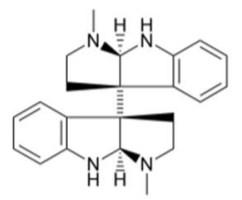

(+)-chimonanthine - H<smiles>CNCCc1cn(C2(c3ccccc3)NCCN2C)c2ccccc12</smiles>

psychohenin

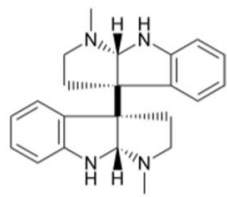

(-)-chimonanthine

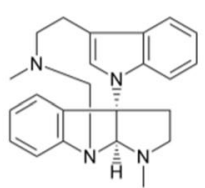

"compound 1"

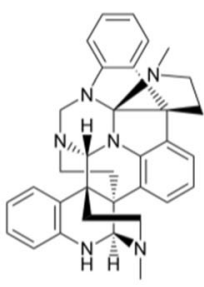

psychotripine

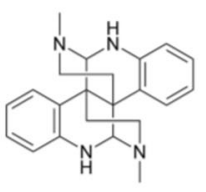

calycanthine

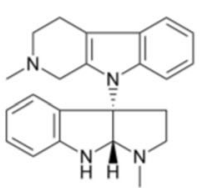

"compound 2"

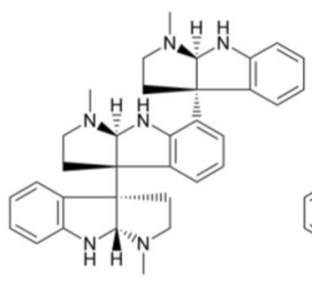

hodgkinsine

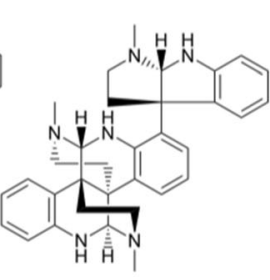

calycosidine

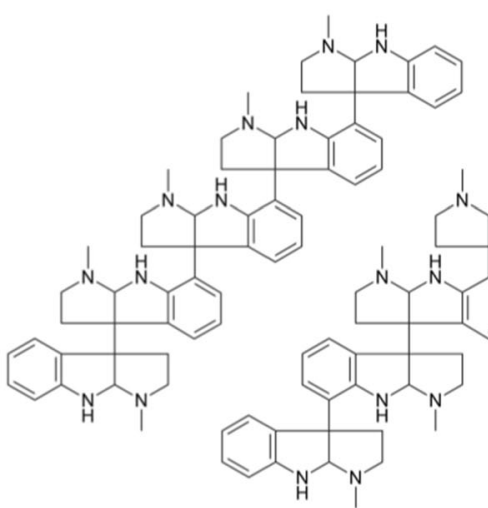

isopsychotridine D

psychotridine C
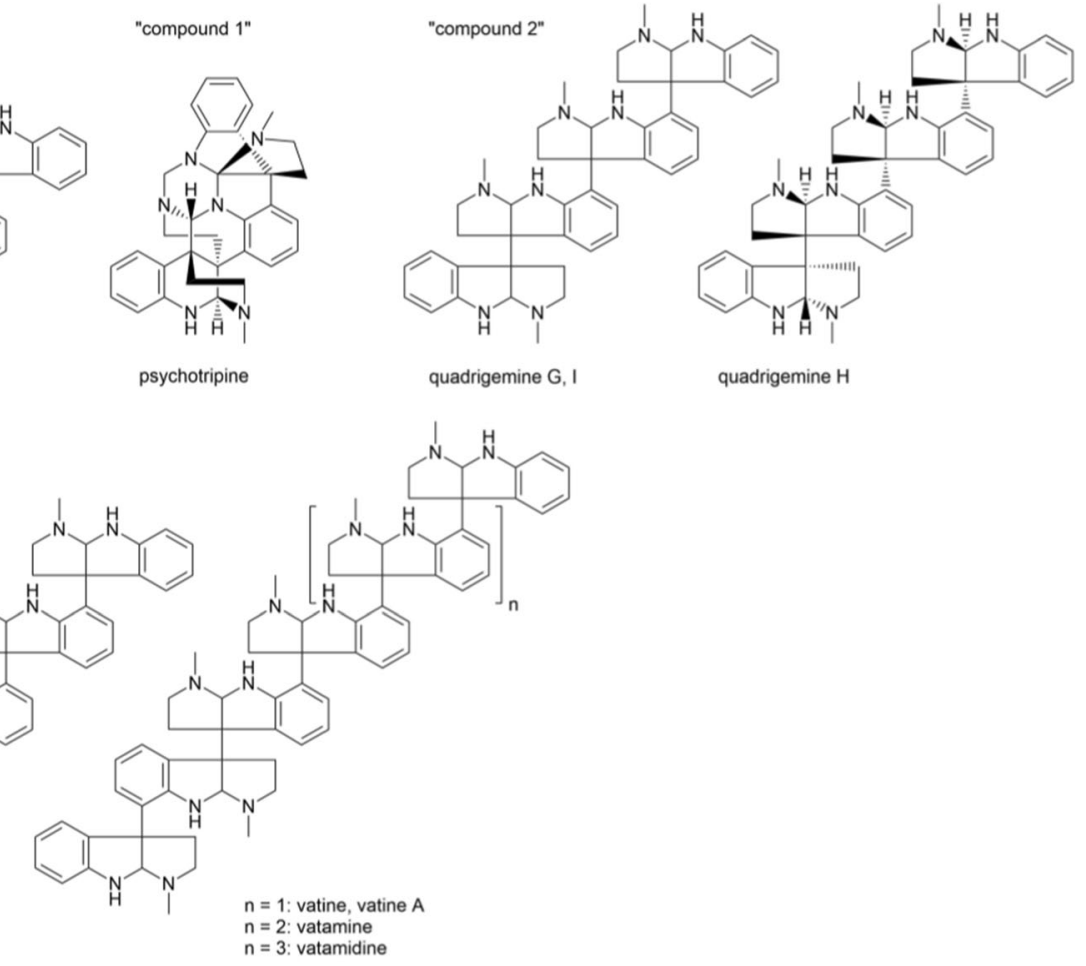

Fig. 17 Alkaloids isolated from Psychotria species, II. Polypyrroloindoline alkaloid dimers to octamers. Note the unusual C-Nlinkage in some of the dimers. Oligomers aligned to the central chimonanthine core<smiles>CN1CCc2c([nH]c3ccccc23)C1</smiles>

\section{2-methyl-tetrahydro} $\beta$-carboline

Fig. 18 Alkaloids isolated from Psychotria species, III. A $\beta$ carboline and a $\gamma$-carboline alkaloid<smiles>CN1CCc2[nH]c3ccccc3c2C1</smiles>

\section{3-methyl-tetrahydro $\mathrm{y}$-carboline}

and feature a methyl group or an oxidized $\mathrm{C}_{1}$ substituent at $C$-3 (Schmidt et al. 2012). $N$-methylcarbazole was never before isolated from plants, and differs from known plant-derived carbazoles by the lack of the $\mathrm{C}_{1}$-substituent at $C$-3. Therefore, its report from Psychotria pilifera is doubtful and is here referred to environmental pollution or contaminated solvents used during the extraction and/or isolation process. 


\section{Conclusion}

Numerous phytochemical studies have been published on species originally ascribed to the genus Psychotria (Psychotrieae). However, recent phylogenetic and morphological data has challenged the traditional circumscription of the genus, which led to the recognition of various segregates within the new tribe Palicoureeae. Based on these revised taxonomic concepts, the phytochemistry of Palicoureeae and Psychotrieae is reviewed here, and accumulation patterns are delineated for most of the genera of the alliance. The present review highlights that alkaloid occurrence in Psychotria is rather limited and excludes all monoterpene indole alkaloids from the genus. Furthermore, it shows that most reports on alkaloids pertain to species of Palicourea and that all genera included in the tribe Palicoureeae feature chemically distinct alkaloid patterns, which may be of ecological relevance such as in plant defence against herbivores.

Electronic supplementary material The online version of this article (https://doi.org/10.1007/s11101-021-09769-x) contains supplementary material, which is available to authorized users.

Acknowledgements We wish to thank the anonymous reviewers for their numerous useful comments that have greatly improved the present article.
Funding Open access funding provided by University of Vienna.

\section{Declarations}

Conflicts of interest The author declared that there is no conflict of interest.

Infomed consent All authors consented to participate in the work.

Open Access This article is licensed under a Creative Commons Attribution 4.0 International License, which permits use, sharing, adaptation, distribution and reproduction in any medium or format, as long as you give appropriate credit to the original author(s) and the source, provide a link to the Creative Commons licence, and indicate if changes were made. The images or other third party material in this article are included in the article's Creative Commons licence, unless indicated otherwise in a credit line to the material. If material is not included in the article's Creative Commons licence and your intended use is not permitted by statutory regulation or exceeds the permitted use, you will need to obtain permission directly from the copyright holder. To view a copy of this licence, visit http://creativecommons.org/licenses/by/4.0/.

\section{Appendix}

See Table 11

Table 11 Compilation of published alkaloids and other compound groups from species of Palicoureeae and Psychotrieae based upon a revised generic classification

\begin{tabular}{|c|c|c|c|}
\hline Accepted species & Alkaloids & Compounds and/or compound groups & References (synonyms) \\
\hline \multicolumn{4}{|l|}{ PALICOUREEAE } \\
\hline \multicolumn{4}{|l|}{ Carapichea } \\
\hline \multirow[t]{2}{*}{ Carapichea affinis } & TIQA & $\begin{array}{l}\text { - Cephaeline, emetine, ipecoside, 6- } O \text {-methyl } \\
\text { ipecoside, borucoside, 6- } O \text {-methyl trans- } \\
\text { cephaeloside }\end{array}$ & Bernhard et al. (2011) \\
\hline & TIQA & $\begin{array}{l}\text { - 7-O-Methyl alangine, ipecoside, 6- } O \text {-methyl } \\
\text { ipecoside, borucoside, } 3^{\prime \prime \prime} \text { - } O \text {-demethyl } \\
\text { borucoside, 6-O-methyl trans-cephaeloside }\end{array}$ & Kornpointner et al. (2018) \\
\hline \multirow[t]{4}{*}{ Carapichea ipecacuanha } & TIQA & $\begin{array}{l}\text { - Emetine, emetamine, protoemetine, } O \text {-methyl } \\
\text { psychotrine }\end{array}$ & $\begin{array}{l}\text { Battersby et al. (1959), Battersby and } \\
\text { Harper (1959, both as "Ipecacuanha } \\
\text { root") }\end{array}$ \\
\hline & TIQA & $\begin{array}{l}\text { - Emetine, cephaeline, emetamine, psychotrine, } \\
O \text {-methyl psychotrine, isoemetine }\end{array}$ & $\begin{array}{l}\text { Hatfield et al. (1981, as Ceph. } \\
\text { ipecacuanha) }\end{array}$ \\
\hline & TIQA & $\begin{array}{l}\text { - Emetine, cephaeline, emetamine, psychotrine, } \\
O \text {-methyl psychotrine, ipecoside, protoemetine }\end{array}$ & $\begin{array}{l}\text { Wiegrebe et al. (1984, as Ceph. } \\
\text { acuminata, Psy. granadensis sensu } \\
\text { auct. non Benth., Psy. ipecacuanha) }\end{array}$ \\
\hline & TIQA & $\begin{array}{l}\text { - Ipecoside, neoipecoside, 7-O-methyl } \\
\text { neoipecoside }\end{array}$ & $\begin{array}{l}\text { Itoh et al. (1989, as Ceph. } \\
\text { ipecacuanha) }\end{array}$ \\
\hline
\end{tabular}


Table 11 continued

\begin{tabular}{|c|c|c|c|}
\hline Accepted species & Alkaloids & Compounds and/or compound groups & References (synonyms) \\
\hline & TIQA & $\begin{array}{l}\text { - Ipecoside, 6- } O \text {-methyl ipecoside, ipecosidic } \\
\text { acid, neoipecoside, 7-O-methyl neoipecoside, } \\
\text { 3,4-dehydro neoipecoside, alangiside, } \\
\text { demethylalangiside; others: iridoids }\end{array}$ & $\begin{array}{l}\text { Itoh et al. (1991, as Ceph. } \\
\text { ipecacuanha) }\end{array}$ \\
\hline & TIQA & - Trans-cephaeloside, cis-cephaeloside & $\begin{array}{l}\text { Nagakura et al. (1993, as Ceph. } \\
\text { ipecacuanha) }\end{array}$ \\
\hline & TIQA & $\begin{array}{l}\text { - Alangiside, 3-O-demethyl-2- } O \text {-methyl } \\
\text { alangiside, 7-O-methyl ipecoside }\end{array}$ & $\begin{array}{l}\text { Itoh et al. (1994, as Ceph. } \\
\text { ipecacuanha) }\end{array}$ \\
\hline & TIQA & $\begin{array}{l}\text { - Emetine, cephaeline, } 7^{\prime} \text { - } O \text {-demethyl } \\
\text { cephaeline, } 10-O \text {-demethyl cephaeline, } 2^{\prime}-N \text { - } \\
\left(1^{\prime \prime} \text {-deoxy- } 1^{\prime \prime}-\beta \text {-D-fructopyranosyl }\right) \text { cephaeline, } \\
\text { isocephaeline, neocephaeline, } 2^{\prime}-N \text { - }\left(1^{\prime \prime} \text {-deoxy- }\right. \\
1^{\prime \prime} \text { - } \beta \text {-D-fructopyranosyl neocephaeline, } \\
\text { psychotrine, protoemetine, 9-demethyl } \\
\text { protoemetinol }\end{array}$ & Itoh et al. (1999, as Ceph. acuminata) \\
\hline & TIQA & $\begin{array}{l}\text { - Demethylalangiside, } 2-O \text { - } \beta \text {-D-glucopyranosyl } \\
\text { demethylalangiside, demethyl-iso-alangiside, } \\
\text { ipecoside, } 6^{\prime \prime}-O-\alpha \text {-D-glucopyranosyl ipecoside, } \\
6^{\prime \prime}-O \text { - } \beta \text {-D-glucopyranosyl ipecoside, }(4 R)-4- \\
\text { hydroxy ipecoside; others: iridoids }\end{array}$ & Itoh et al. (2002, as Ceph. acuminata) \\
\hline Carapichea klugii & TIQA & $\begin{array}{l}\text { - Cephaeline, isocephaeline, } 7^{\prime} \text { - } O \text {-demethyl } \\
\text { isocephaeline, klugine, } 7-O \text {-methyl ipecoside }\end{array}$ & $\begin{array}{l}\text { Muhammad et al. (2003, as Psy. } \\
\text { klugii) }\end{array}$ \\
\hline \multicolumn{4}{|l|}{ Chassalia } \\
\hline Chassalia capitata & n.s. ${ }^{1}$ & - Others: flavonoids & Soobrattee et al. (2005) \\
\hline Chassalia coriacea & n.s. ${ }^{1}$ & - Others: flavonoids & Soobrattee et al. (2005) \\
\hline \multirow[t]{2}{*}{ Chassalia curviflora } & MIA & - Alstrostine A & Schinnerl et al. (2012) \\
\hline & n.d & - Others: coumarins, polyphenols & Wang and Zhou (1999) \\
\hline Chassalia grandifolia & n.s. ${ }^{1}$ & - Others: flavonoids & Soobrattee et al. (2005) \\
\hline Chassalia lanceolata & n.s. ${ }^{1}$ & - Others: flavonoids & Soobrattee et al. (2005) \\
\hline Chassalia petrinensis & n.s. ${ }^{1}$ & - Others: flavonoids & Soobrattee et al. (2005) \\
\hline \multicolumn{4}{|l|}{ Eumachia } \\
\hline Eumachia cymuligera & PIA & - Hodgkinsine, quadrigemine B & Brand et al. (2012) \\
\hline \multirow[t]{2}{*}{ Eumachia depauperata } & PIA & $\begin{array}{l}\text { - Calycosidine, } N-8^{\prime \prime} \text {-formyl calycosidine, } N-8^{\prime \prime} \text { - } \\
\text { methyl- } N-1^{\prime} \text {-demethyl iso-calycosidine, } \\
\text { hodgkinsine }\end{array}$ & $\begin{array}{l}\text { Nascimento et al. (2015b, as Marg. } \\
\text { carrascoana) }\end{array}$ \\
\hline & n.s & - Others: flavonoids, polyphenols & $\begin{array}{l}\text { Nascimento et al. (2015a, as Marg. } \\
\text { carrascoana) }\end{array}$ \\
\hline \multirow[t]{2}{*}{ Eumachia forsteriana } & PIA & $\begin{array}{l}\text { - (-)-Calycanthine, iso-calycanthine, meso- } \\
\text { chimonanthine }\end{array}$ & $\begin{array}{l}\text { Adjibadé et al. } \\
\text { (1985, 1986, 1989, 1992, all as Psy. } \\
\text { forsteriana) }\end{array}$ \\
\hline & PIA & $\begin{array}{l}\text { - Quadrigemine A, quadrigemine B, } \\
\text { psychotridine, isopsychotridine C }\end{array}$ & $\begin{array}{l}\text { Roth et al. (1985, as Psy. forsteriana), } \\
\text { see also Jamison et al. (2017) }\end{array}$ \\
\hline \multirow[t]{2}{*}{ Eumachia frutescens } & PIA & - Hodgkinsine & $\begin{array}{l}\text { Anet et al. (1961, as Hod. frutescens); } \\
\text { Fridrichsons et al. (1967, 1974, as } \\
\text { Hod. frutescens) }\end{array}$ \\
\hline & PIA & - Quadrigemine A, quadrigemine B & Parry et al. (1978, as Hod. frutescens) \\
\hline Eumachia leptothyrsa & PIA & - Psychotridine & Hart et al. (1974, as Psy. beccarioides) \\
\hline Eumachia lyciiflora & PIA & $\begin{array}{l}\text { - Meso-chimonanthine, } N_{b} \text {-desmethyl meso- } \\
\text { chimonanthine, hodgkinsine }\end{array}$ & Jannic et al. (1999, as Psy. lyciiflora) \\
\hline Eumachia oleoides & PIA & $\begin{array}{l}\text { - Hodgkinsine, quadrigemine C, psychotridine, } \\
\text { isopsychotridine A, isopsychotridine B }\end{array}$ & $\begin{array}{l}\text { Libot et al. (1987, as Psy. oleoides); } \\
\text { see also Jamison et al. (2017) }\end{array}$ \\
\hline
\end{tabular}


Table 11 continued

\begin{tabular}{lll}
\hline Accepted species & Alkaloids & Compounds and/or compound groups \\
\hline PIA & - Hodgkinsine, psycholeine, quadrigemine C \\
& PIA & $\begin{array}{l}\text { - Hodgkinsine, quadrigemine C, psychotridine, } \\
\text { isopsychotridine B, quadrigemine I, oleoidine, } \\
\text { caledonine }\end{array}$ \\
& n.d & - Others: coumarins, flavonoids, triterpenes \\
Eumachia straminea & PIA & $-(-)$-Calycanthine, $(+)$-chimonanthine, \\
& calycosidine, hodgkinsine, quadrigemine B & - Psychotrimine, psychopentamine
\end{tabular}

Geophila

Geophila macropoda

Geophila uniflora

MIA

n.d

Notopleura

Notopleura camponutans

Notopleura polyphlebia

Notopleura uliginosa

Palicourea

Palicourea acuminata

Palicourea adusta

Palicourea alpina

Palicourea axillaris

Palicourea brachypoda

Palicourea chiriquensis

MIA

Palicourea colorata n.d

n.s

n.d - Others: quinones

MIA

MIA

$\beta C A$, MIA

PIA - Calycanthine

n.d - Others: megastigmanes

MIA - $(E)-O-\left(6^{\prime}\right)-\left(4^{\prime \prime}-\right.$-Hydroxy- $3^{\prime \prime}$-methoxy)cinnamoyl lyaloside, $(E)$-vallesiachotamine, (Z)-vallesiachotamine

- Psychollatine, umbellatine

Alstrostines triterpenes

- Others: essential oils

- Others: diterpenoids

- Others: megastigmanes, flavonoids, quinones

- Others: megastigmanes, quinones desoxycordifoline, lagamboside, lyaloside, strictosamide; others: coumarins

Lyaloside, $(E)-O-\left(6^{\prime}\right)-\left(4^{\prime \prime}\right.$-hydroxy- $3^{\prime \prime}$ methoxy)-cinnamoyl lyaloside, $(E)-O-\left(6^{\prime}\right)-\left(4^{\prime \prime}\right.$ lyaloside

- Harman, palinine

- Psychollatine, 3,4-dehydro-18,19- $\beta$-epoxy psychollatine, $N^{4}$-[1-((R)-2-hydroxypropyl $\left.)\right]$ psychollatine, $N^{4}$-[1-((S)-2-hydroxypropyl)] psychollatine

- 5 $\alpha$-Carboxystrictosidine, lyaloside; others: iridoids

PIA - (-)-Calycanthine, iso-calycanthine, (+)chimonanthine, PML $100\left(=8-8 \mathrm{a}, 8^{\prime}-8^{\prime} \mathrm{a}\right.$
References (synonyms)

Guéritte-Voegelein et al. (1992);

Rasolonjanahary et al. (1995, both as

Psy. oleoides)

Jannic et al. (1999, as Psy. oleoides); see also Jamison et al. (2017)

Fu et al. (2015, as Psy. straminea)

Lajis et el. (1993); Mahmud et al. (1993, both as Psy. rostrata)

Takayama et al. (2004, as Psy. rostrata)

Berger et al., unpublished

Luo et al. (2011, as Geo. herbacea)

Rao et al. (2017, misidentified as Geo. repens)

Dash et al. (2019, misidentified as Geo. repens)

Jacobs et al. (2008); Solís et al. (1995, both as Psy. camponutans)

Berger (2012); Berger et al. (2016)

Kostyan (2017)

Berger (2012); Berger et al. (2012, 2017)

Valverde et al. (1999)

Stuart and Woo-Ming (1974)

Woo-Ming and Stuart (1975)

Stuart and Woo-Ming (1975)

Martín et al. (1994, as Ceph. axillaris)

Both et al. (2002); Kerber et al. (2008, as Psy. umbellata Vell.)

Kerber et al. (2014, as Psy. umbellata Thonn.)

Berger (2012, as Psy. chiriquensis)

Verotta et al. (1998, as Psy. colorata) 
Table 11 continued

\begin{tabular}{|c|c|c|c|}
\hline \multirow[t]{2}{*}{ Accepted species } & Alkaloids & $\begin{array}{l}\text { Compounds and/or compound groups } \\
\left.\text { tetradehydro iso-calycanthine } 3 \mathrm{a}(R), 3^{\prime} \mathrm{a}(R)\right) \text {, } \\
\text { hodgkinsine, quadrigemine } \mathrm{C}\end{array}$ & References (synonyms) \\
\hline & PIA & $\begin{array}{l}\text { - Hodgkinsine, quadrigemine } \mathrm{B} \text {, quadrigemine } \mathrm{C} \text {, } \\
\text { psychotridine }\end{array}$ & Verotta et al. (1999, as Psy. colorata) \\
\hline \multirow[t]{4}{*}{ Palicourea coriacea } & $\begin{array}{r}\text { MIA, } \\
\text { PIA }\end{array}$ & $\begin{array}{l}\text { - Calycanthine, strictosidinic acid, 3-epi- } \\
\text { strictosidinic acid, strictosidinic ketone; others: } \\
\text { triterpenes }\end{array}$ & do Nascimento et al. (2006) \\
\hline & MIA & $-4^{\prime}-O-3^{\prime \prime}$-Sucrose-strictosidinic acid & do Nascimento et al. (2008) \\
\hline & PIA & $\begin{array}{l}\text { - Calycanthine; others: polyphenols, quinones, } \\
\text { triterpenes }\end{array}$ & da Silva et al. (2008) \\
\hline & PIA & - Calycanthine & Kato et al. (2017) \\
\hline Palicourea correae & MIA & $\begin{array}{l}\text { - 10-Hydroxy correantoside, correantine A, } \\
\text { correantine B, correantine C, 20-epi- } \\
\text { correantine B, correantoside, } \\
\text { isodolichantoside; others: megastigmanes, } \\
\text { tetraterpenes }\end{array}$ & $\begin{array}{l}\text { Achenbach et al. (1995, as Psy. } \\
\text { correae) }\end{array}$ \\
\hline \multirow[t]{4}{*}{ Palicourea crocea } & MIA & - Croceaine A, croceaine B, & Düsman et al. (2004) \\
\hline & MIA & $\begin{array}{l}\text { - Croceaine A, psychollatine, } 3,4- \\
\text { dehydropsychollatin } \mathrm{N}^{2} \text {-oxide }(=3,4 \text {-dihydro- } \\
\text { 1-(1- } \beta \text {-D-glucopyranosyloxy-1,4a,5,7a- } \\
\text { tetrahydro-4- } \\
\text { methoxycarbonylcyclopenta[c]pyran-7-yl)- } \beta \text { - } \\
\left.\text { carboline- } N^{2} \text {-oxide }\right)\end{array}$ & Narine and Maxwell (2009) \\
\hline & MIA & $\begin{array}{l}\text { - Brachycerine, palicroceaine, strictosidine, } \\
\text { strictosidinic acid }\end{array}$ & Berger et al. (2015) \\
\hline & n.s & - Others: flavonoids & Berger et al. (2016) \\
\hline Palicourea croceoides & MIA & - Strictosidine, strictosidinic acid & Berger et al. (2015) \\
\hline Palicourea cyanococca & MIA & $\begin{array}{l}\text { - Bahienoside B, } 5 \alpha \text {-carboxystrictosidine, } \\
\text { desoxycordifoline, lagamboside, lyaloside }\end{array}$ & $\begin{array}{l}\text { Berger (2012, as Psy. cyanococca), } \\
\text { Berger et al. (2017) }\end{array}$ \\
\hline Palicourea deflexa & $\begin{array}{l}\beta C A, \\
\text { MIA }\end{array}$ & - Harman-3-carboxylic acid, strictosidinic acid & $\begin{array}{l}\text { Bertelli et al. }(2015,2017 \text {, both as Psy. } \\
\text { deflexa) }\end{array}$ \\
\hline \multirow[t]{2}{*}{ Palicourea demissa } & n.d & - Others: coumarins, polyphenols, triterpenes & El-Seedi (1999) \\
\hline & n.d & - Others: coumarins, flavonoids, triterpenes & Sosa Moreno (2011) \\
\hline Palicourea dichroa & MIA & $\begin{array}{l}\text { - Angustine, strictosidine, strictosamide, }(E) \text { - } \\
\text { vallesiachotamine, vallesiachotamine lactone }\end{array}$ & Solis et al. (1993, as Ceph. dichroa) \\
\hline Palicourea domingensis & PIA & - Chimonanthine & Ripperger (1982) \\
\hline \multirow[t]{2}{*}{ Palicourea elata } & MIA & - Strictosidine & Berger $(2012$, as Psy. elata $)$ \\
\hline & n.s & - Others: chlorogenic acids & Berger et al. (2016) \\
\hline Palicourea eurycarpa & n.s & - Others: essential oils & Setzer et al. (2006) \\
\hline Palicourea fastigiata & MIA & - Brachycerine & Berger et al. (2015) \\
\hline Palicourea garciae & MIA & - Palicoside & Berger et al. (2017) \\
\hline Palicourea glomerulata & PIA & $\begin{array}{l}\text { - Glomerulatine A, glomerulatine B, } \\
\text { glomerulatine C }\end{array}$ & Solis et al. (1997, as Psy. glomerulata) \\
\hline Palicourea gracilenta & TA & $\begin{array}{l}\text { - Bufotenin, brachybotryne, } N \text {-oxo } \\
\text { brachybotryne }\end{array}$ & $\begin{array}{l}\text { Ribeiro et al. (2016, as Psy. } \\
\text { brachybotrya) }\end{array}$ \\
\hline \multirow[t]{2}{*}{$\begin{array}{l}\text { Palicourea } \\
\text { hoffmannseggiana }\end{array}$} & $\begin{array}{l}\beta C A, \\
\text { MIA }\end{array}$ & - Harman, strictosidinic acid & $\begin{array}{l}\text { de Oliveira et al. (2013, as. Psy. } \\
\text { barbiflora) }\end{array}$ \\
\hline & $\begin{array}{l}\text { TA, } \\
\text { ßCA, } \\
\text { MIA, } \\
\text { PIA }\end{array}$ & $\begin{array}{l}\text { - } N \text {-Methyltryptamine, harman, 2-methyl } \\
\text { tetrahydro- } \beta \text {-carboline, }(+) \text {-chimonanthine, } \\
\text { strictosidinic acid; others: coumarins, } \\
\text { polyphenols }\end{array}$ & Naves (2014) \\
\hline
\end{tabular}


Table 11 continued

\begin{tabular}{|c|c|c|c|}
\hline Accepted species & Alkaloids & Compounds and/or compound groups & References (synonyms) \\
\hline Palicourea luxurians & MIA & $\begin{array}{l}\text { - Alstrostine A, dehydro-rudgeifoline, iso- } \\
\text { alstrostine A, } 5 \alpha \text {-carboxystrictosidine, } \\
\text { javaniside; others: iridoids }\end{array}$ & Kornpointner et al. (2020) \\
\hline Palicourea longiflora & n.d & - Others: polyphenols & Coelho et al. (2007) \\
\hline \multirow[t]{2}{*}{ Palicourea mamillaris } & MIA & - Myrianthosine, strictosidinic acid & $\begin{array}{l}\text { Simões-Pires et al. (2006, as Psy. } \\
\text { myriantha) }\end{array}$ \\
\hline & MIA & - Strictosidinic acid & Farias et al. (2012, as Psy. myriantha) \\
\hline \multirow[t]{2}{*}{ Palicourea marcgravii } & MIA & - Palicoside & Morita et al. (1989) \\
\hline & $\mathrm{PA}, \beta \mathrm{CA}$ & $\begin{array}{l}\text { - } N \text {-Methyltyramine, } 2 \text {-methyl tetrahydro- } \beta \text { - } \\
\text { carboline }\end{array}$ & Kemmerling (1996) \\
\hline Palicourea minutiflora & MIA & $\begin{array}{l}\text { - Strictosidinic acid, vincosamide; others: } \\
\text { iridoids, polyphenols, triterpenes }\end{array}$ & Moura et al. (2020a, 2020b) \\
\hline Palicourea mortoniana & n.d & - Others: flavonoids, chlorogenic acids & $\begin{array}{l}\text { Berger et al. (2016, as Psy. } \\
\text { mortoniana) }\end{array}$ \\
\hline \multirow[t]{2}{*}{ Palicourea muscosa } & PIA & $\begin{array}{l}\text { - (-)-Calycanthine, }(+) \text {-chimonanthine, meso- } \\
\text { chimonanthine, PML } 100\left(=8-8 \mathrm{a}, 8^{\prime}-8^{\prime} \mathrm{a}\right. \\
\left.\text { tetradehydro iso-calycanthine } 3 \mathrm{a}(R), 3^{\prime} \mathrm{a}(R)\right) \text {, } \\
\text { PML } 300, \text { hodgkinsine }\end{array}$ & Verotta et al. (1999, as Psy. muscosa) \\
\hline & PIA & - Hodgkinsine, quadrigemine $\mathrm{H}$ & Jamison et al. (2017, as Psy. muscosa) \\
\hline Palicourea ovalis & PIA & - Calycanthine & Garcia et al. (1997) \\
\hline \multirow[t]{2}{*}{ Palicourea padifolia } & MIA & $\begin{array}{l}\text { - Lyaloside, }(E)-O-\left(6^{\prime}\right)-\left(4^{\prime \prime} \text {-hydroxy- } 3^{\prime \prime}, 5^{\prime \prime} \text { - }\right. \\
\text { dimethoxy)-cinnamoyl lyaloside, strictosidine }\end{array}$ & Berger et al. (2015) \\
\hline & n.s & - Others: flavonoids & Berger et al. (2016) \\
\hline \multirow[t]{3}{*}{ Palicourea prunifolia } & MIA & - Strictosamide, prunifoleine, 14-oxoprunifoleine & Faria et al. (2010, as Psy. prunifolia) \\
\hline & MIA & $\begin{array}{l}\text { - Strictosamide, 10-hydroxy isodeppeaninol, } \\
\text { 10-hydroxy antirhine, 10-hydroxy antirhine } \mathrm{N} \text { - } \\
\text { oxide }\end{array}$ & Kato et al. (2012, as Psy. prunifolia) \\
\hline & MIA & $\begin{array}{l}\text { - Prunifoleine, 10-hydroxy antirhine, 10-hydroxy } \\
\text { isodeppeaninol }\end{array}$ & Kato et al. (2017, as Psy. prunifolia) \\
\hline Palicourea racemosa & n.d & - Others: flavonoids & Berger (2012); Berger et al. (2016) \\
\hline \multirow[t]{10}{*}{ Palicourea rigida } & n.d & - Others: triterpenes & Bolzani et al. (1992) \\
\hline & n.d & - Others: iridoids & Lopes et al. (2004) \\
\hline & MIA & - Vallesiachotamine & Vencato et al. (2006) \\
\hline & n.d & - Others: flavonoids & da Rosa et al. (2010) \\
\hline & n.d & - Others: iridoids & de Freitas Morel et al. (2011) \\
\hline & n.d & - Others: iridoids & da Silva et al. (2013) \\
\hline & n.d & - Others: diterpenes, triterpenes, iridoids & Alves et al. (2016) \\
\hline & n.d & - Others: iridoids & Valdevite et al. (2016) \\
\hline & n.d & - Others: coumarins & Alves et al. (2017) \\
\hline & n.d & - Others: flavonoids & Pinheiro et al. (2018) \\
\hline Palicourea semirasa & PIA & - Calycanthine, chimonanthine & $\begin{array}{l}\text { Nakano and Martín (1976, as Pal. } \\
\text { fendleri) }\end{array}$ \\
\hline \multirow[t]{2}{*}{ Palicourea sessilis } & n.d & - Others: coumarins, triterpenes & $\begin{array}{l}\text { Moreno et al. (2014, as Psy. } \\
\text { vellosiana) }\end{array}$ \\
\hline & TA, MIA & $\begin{array}{l}\text { - Alline, } N \text {-methyltryptamine, } 4-N \text {-methyl } \\
\text { lyaloside, } 4-N \text {-methyl-3,4-dehydro } \\
\text { strictosidine, isodolichantoside, } 4 \alpha \text {-hydroxy }\end{array}$ & Klein-Júnior et al. (2017) \\
\hline
\end{tabular}


Table 11 continued

\begin{tabular}{|c|c|c|c|}
\hline \multirow[t]{2}{*}{ Accepted species } & Alkaloids & $\begin{array}{l}\text { Compounds and/or compound groups } \\
\text { isodolichantoside, } 4 \beta \text {-hydroxy } \\
\text { isodolichantoside, } 5 \text {-oxodolichantoside }\end{array}$ & References (synonyms) \\
\hline & n.d & $\begin{array}{l}\text { - Others: hydroxycinnamic acid amides, } \\
\text { flavonoids, polyphenols }\end{array}$ & Samulski et al. (2020) \\
\hline Palicourea spectabilis & n.d & - Others: diterpenes, coumarines, flavonoids & $\begin{array}{l}\text { Benevides et al. (2005, as Psy. } \\
\text { spectabilis) }\end{array}$ \\
\hline Palicourea didymocarpos & MIA & $\begin{array}{l}\text { - Bahienoside A, bahienoside B, } 5 \alpha \text { - } \\
\text { carboxystrictosidine, angustine, strictosamide, } \\
\text { (E)-vallesiachotamine, }(Z) \text {-vallesiachotamine }\end{array}$ & $\begin{array}{l}\text { Paul et al. (2003, misidentified as Psy. } \\
\text { bahiensis) }\end{array}$ \\
\hline \multirow[t]{2}{*}{ Palicourea suerrensis } & $\beta \mathrm{CA}$ & - Harman & $\begin{array}{l}\text { Murillo and Castro (1998, as Psy. } \\
\text { suerrensis) }\end{array}$ \\
\hline & MIA & $\begin{array}{l}\text { - Lyaloside, lyalosidic acid, strictosidine, } \\
\text { strictosidinic acid, ophiorine A, ophiorine B; } \\
\text { others: coumarines }\end{array}$ & $\begin{array}{l}\text { Berger (2012, as Psy. suerrensis); } \\
\text { Berger et al. (2017) }\end{array}$ \\
\hline Palicourea tsakiana & MIA & - Palicoside & Berger et al. (2017) \\
\hline Palicourea winkleri & $\begin{array}{l}\beta C A, \\
\text { MIA }\end{array}$ & $\begin{array}{l}\text { - Tetrahydronorharman-1-one, lyalosidic acid, } \\
\text { strictosamide, deoxostrictosamide, } \\
\text { strictosidinic acid }\end{array}$ & Berger et al. (2017) \\
\hline Psychotria brachyceras $^{2}$ & MIA & - Brachycerine & Kerber et al. (2001) \\
\hline \multirow[t]{3}{*}{ Psychotria laciniata $^{2}$} & MIA & $\begin{array}{l}\text { - Lyaloside, }(E)-O-\left(6^{\prime}\right)-\left(4^{\prime \prime} \text {-hydroxy- } 3^{\prime \prime}, 5^{\prime \prime}-\right. \\
\text { dimethoxy)-cinnamoyl lyaloside, } \\
\text { strictosamide, }(E) \text {-vallesiachotamine, }(Z) \text { - } \\
\text { vallesiachotamine (= isovallesiachotamine) }\end{array}$ & dos Santos et al. (2013a) \\
\hline & MIA & $\begin{array}{l}\text { - Angustine, vallesiachotamine lactone, }(E) \text { - } \\
\text { vallesiachotamine, }(Z) \text {-vallesiachotamine, } \\
\text { pauridianthoside }\end{array}$ & dos Santos et al. (2013b) \\
\hline & MIA & $\begin{array}{l}\text { - Lyaloside, }(E)-O-\left(6^{\prime}\right)-\left(4^{\prime \prime} \text {-hydroxy- } 3^{\prime \prime}, 5^{\prime \prime} \text { - }\right. \\
\text { dimethoxy)-cinnamoyl lyaloside, } \\
\text { pauridianthoside, strictosamide, } \\
\text { vallesiachotamine lactone, }(E) \text { - } \\
\text { vallesiachotamine, }(Z) \text {-vallesiachotamine } \\
\text { (= isovallesiachotamine) }\end{array}$ & $\begin{array}{l}\text { Queiroz et al. (2017, as Psy. } \\
\text { stenocalyx) }\end{array}$ \\
\hline \multirow[t]{5}{*}{ Psychotria leiocarpa $^{2}$} & n.s & - Others: iridoids & Lopes et al. (2004) \\
\hline & MIA & - Strictosamide & Lopes (1998) \\
\hline & MIA & - $N, \beta$-D-Glucopyranosyl vincosamide & Henriques et al. (2004) \\
\hline & MIA & - $N, \beta$-D-Glucopyranosyl vincosamide & Matsuura and Fett-Neto (2013) \\
\hline & n.s & - Others: essential oils & Andrade et al. (2010) \\
\hline Psychotria nemorosa $^{2}$ & $\begin{array}{l}\text { PA, TA, } \\
\beta C A \\
\text { MIA }\end{array}$ & $\begin{array}{l}\text { - Hordenine, } N \text {-formyltryptamine, } 2 \text {-methyl } \\
\text { tetrahydro- } \beta \text {-carboline, strictosidine; others: } \\
\text { fatty acids, iridoids, polyphenols, triterpenes }\end{array}$ & Calixto et al. (2017) \\
\hline \multirow[t]{2}{*}{ Psychotria nuda ${ }^{2}$} & MIA & - Strictosamide & Farias et al. (2008) \\
\hline & TA, MIA & $\begin{array}{l}\text { - N, } N, N \text {-Trimethyltryptamine, strictosidine, } 5 \alpha- \\
\text { carboxystrictosidine, strictosamide, lyaloside; } \\
\text { others: coumarines, iridoids, polyphenols, } \\
\text { triterpenes }\end{array}$ & de Carvalho Junior et al. (2019) \\
\hline \multirow[t]{3}{*}{ Psychotria stachyoides $^{2}$} & MIA & - Stachyoside, nor-methyl-23-oxo-correantoside & Pimenta et al. (2010a) \\
\hline & MIA & - Correantosine E, correantosine F & Pimenta et al. (2010b) \\
\hline & MIA & $\begin{array}{l}\text { - } N \text {-Demethylcorreantoside; others: coumarines, } \\
\text { quinones, triterpenes }\end{array}$ & Pimenta et al. (2011) \\
\hline Psychotria suterella ${ }^{2}$ & MIA & - Lyaloside, strictosamide, naucletine & van de Santos et al. (2001) \\
\hline
\end{tabular}


Table 11 continued

\begin{tabular}{|c|c|c|c|}
\hline Accepted species & Alkaloids & Compounds and/or compound groups & References (synonyms) \\
\hline & MIA & $\begin{array}{l}\text { - Lyaloside, }(E)-O-\left(6^{\prime}\right)-\left(4^{\prime \prime} \text {-hydroxy- } 3^{\prime \prime}, 5^{\prime \prime}-\right. \\
\text { dimethoxy)-cinnamoyl lyaloside, } \\
\text { strictosamide, }(E) \text {-vallesiachotamine, }(Z)- \\
\text { vallesiachotamine (= isovallesiachotamine) }\end{array}$ & dos Santos et al. (2013a) \\
\hline & MIA & $\begin{array}{l}\text { - Lyalosidic acid, strictosidinic acid; others: } \\
\text { iridoids, triterpenes }\end{array}$ & de Carvalho Junior et al. (2021) \\
\hline \multicolumn{4}{|l|}{ Rudgea } \\
\hline Rudgea cornifolia & MIA & - Rudgeifoline & Schinnerl et al. (2012) \\
\hline \multirow[t]{2}{*}{ Rudgea jasminoides } & n.d & - Others: triterpenes & Lopes et al. (1999) \\
\hline & n.d & - Others: quinones & de Cacia et al. (2007) \\
\hline Rudgea viburnoides & n.d & - Others: triterpenes & Young et al. (1998) \\
\hline \multicolumn{4}{|l|}{ PSYCHOTRIEAE } \\
\hline \multicolumn{4}{|l|}{ Psychotria s. str } \\
\hline \multirow[t]{4}{*}{ Hydnophytum formicarum $^{2}$} & n.d & - Others: flavonoids, polyphenols, triterpenes & Rédei et al. (2005) \\
\hline & n.d & - Others: flavonoids, polyphenols, triterpenes & Hasmah et al. (2008) \\
\hline & n.d & - Others: flavonoids, polyphenols, triterpenes & Prachayasittikul et al. $(2008,2012)$ \\
\hline & n.d & - Others: polyphenols, triterpenes & Abdullah et al. (2017a, 2017b) \\
\hline \multirow[t]{2}{*}{ Myrmecodia pendens $^{2}$} & n.d & - Others: flavonoids, polyphenols & Engida et al. $(2013,2015)$ \\
\hline & n.d & - Others: flavonoids, diterpenoids, triterpenoids & $\begin{array}{l}\text { Alibasyah et al. (2017); Gartika et al. } \\
\text { (2018), Kurnia et al. (2019); Satari } \\
\text { et al. (2019) }\end{array}$ \\
\hline Myrmecodia tuberosa $^{2}$ & n.d & - Others: iridoids & Hanh et al. (2016) \\
\hline \multirow[t]{2}{*}{ Psychotria adenophylla } & n.d & - Others: triterpenes & Dan and Dan (1986) \\
\hline & n.d & - Others: polyphenols & Berger (2012) \\
\hline \multirow[t]{4}{*}{ Psychotria asiatica } & n.d & - Others: quinones, sesquiterpenes & Hayashi et al. (1987, as Psy. rubra) \\
\hline & n.s & - Others: iridoids & Inouye et al. (1988, as Psy. rubra) \\
\hline & n.d & $\begin{array}{l}\text { - Others: aliphatic compounds, triterpenes, } \\
\text { amides }\end{array}$ & Giang et al. (2007, as Psy. reevesii) \\
\hline & n.d & - Others: flavonoids, iridoids & Lu et al. (2014a, as Psy. rubra) \\
\hline Psychotria brandneriana & n.d & - Others: polyphenols & $\begin{array}{l}\text { Berger (2012, misidentified as } P \text {. } \\
\text { verschuerenii) }\end{array}$ \\
\hline Psychotria calocarpa & PIA & - Psychotriasine & Zhou et al. (2010) \\
\hline \multirow[t]{2}{*}{ Psychotria capensis } & n.d & - Others: triterpenes, tetraterpenes & Kafua et al. (2009) \\
\hline & n.d & - Others: polyphenols & Berger (2012) \\
\hline \multirow[t]{2}{*}{ Psychotria carthagenensis } & n.d & - Others: triterpenes & $\begin{array}{l}\text { Leal and Elisabetsky (1996), Lopes } \\
\text { et al. (2000); see also Rivier and } \\
\text { Lindgren (1972) }\end{array}$ \\
\hline & n.d & - Others: polyphenols & Berger (2012) \\
\hline Psychotria chagrensis & n.d & - Others: polyphenols & Berger (2012) \\
\hline Psychotria fractistipula & n.d & - Others: triterpenes & de Oliveira (2015) \\
\hline Psychotria gitingensis & n.d & - Others: megastigmanes & Tan et al. (2012) \\
\hline Psychotria graciliflora & n.d & - Others: iridoids, polyphenols & Berger (2012) \\
\hline Psychotria grandis & n.d & - Others: iridoids, polyphenols & Berger (2012) \\
\hline Psychotria hainanensis & n.d & $\begin{array}{l}\text { - Others: aliphatic compounds, flavonoids, } \\
\text { triterpenes }\end{array}$ & Li et al. (2011a) \\
\hline Psychotria hawaiiensis & n.d & - Others: iridoids, polyphenols & Berger (2012) \\
\hline
\end{tabular}


Table 11 continued

\begin{tabular}{|c|c|c|c|}
\hline Accepted species & Alkaloids & Compounds and/or compound groups & References (synonyms) \\
\hline \multirow[t]{2}{*}{ Psychotria henryi } & PIA & - "Compound 1", “compound 2" & Liu et al. (2013) \\
\hline & PIA & - Psychohenin & Liu et al. (2014) \\
\hline Psychotria hexandra & n.d & - Others: polyphenols & Berger (2012) \\
\hline Psychotria horizontalis & n.d & - Others: iridoids, polyphenols & Berger (2012) \\
\hline Psychotria ligustrifolia & n.d & - Others: polyphenols & Berger (2012) \\
\hline Psychotria limonensis & n.d & - Others: polyphenols & Berger (2012) \\
\hline Psychotria luzoniensis & n.d & $\begin{array}{l}\text { - Others: flavonoids, iridoids, megastigmanes, } \\
\text { sugars }\end{array}$ & Ramil et al. (2020) \\
\hline \multirow[t]{2}{*}{ Psychotria malayana $^{4}$} & PIA & - Chimonanthine, hodgkinsine & Hadi and Bremner (2001) \\
\hline & $\begin{array}{l}\text { PIA, } \\
\beta C A\end{array}$ & $\begin{array}{l}\text { - (+)-Chimonanthine, }(-) \text {-chimonanthine, meso- } \\
\text { chimonanthine, calycanthine, hodgkinsine, } \\
\text { 2-methyl tetrahydro- } \gamma \text {-carboline; others: } \\
\text { pyrazine }\end{array}$ & Hadi et al. (2014) \\
\hline Psychotria manillensis & n.s & - Others: iridoids & Inouye et al. (1988) \\
\hline Psychotria marginata & n.d & - Others: polyphenols & Berger (2012) \\
\hline \multirow[t]{2}{*}{ Psychotria mariniana } & n.d & - Others: iridoids, triterpenes & Gonzalez and Dieck (1996) \\
\hline & n.d & - Others: iridoids, polyphenols & Berger (2012) \\
\hline Psychotria micrantha & n.d & - Others: iridoids, polyphenols & Berger (2012) \\
\hline \multirow[t]{3}{*}{ Psychotria milnei } & PIA & - Calycosidine, hodgkinsine & Libot et al. (1987, as Cal. milnei) \\
\hline & PIA & - Vatine, vatine A, vatamine, vatamidine & Adjibadé et al. (1990, as Cal. milnei) \\
\hline & PIA & $\begin{array}{l}\text { - Hodgkinsine, quadrigemine } \mathrm{H} \text {, psychotridine } \mathrm{C} \text {, } \\
\text { isopsychotridine } \mathrm{E} \text {, vatine, vatine A, vatamine, } \\
\text { vatamidine }\end{array}$ & Saad et al. (1995, as Cal. milnei) \\
\hline Psychotria nervosa & n.d & - Others: flavonoids, polyphenols & Berger (2012); Berger et al. (2016) \\
\hline Psychotria orophila & n.d & - Others: polyphenols & Berger (2012) \\
\hline Psychotria orosiana & n.d & - Others: iridoids, polyphenols & Berger (2012) \\
\hline Psychotria parvifolia & n.d & - Others: polyphenols & Berger (2012) \\
\hline Psychotria pilifera & PIA & - Psychotripine & Li et al. (2011b) \\
\hline $\begin{array}{l}\text { some compounds probably } \\
\text { originating from an } \\
\text { adulteration by an } \\
\text { Apocynaceae }\end{array}$ & $\begin{array}{l}\text { PIA, } \\
\beta C A \\
\text { MIA }\end{array}$ & $\begin{array}{l}\text { - Psychotriasine, quadrigemine I, calycanthine, } \\
\text { iso-calycanthine, 2-methyl tetrahydro- } \beta \text { - } \\
\text { carboline; } \\
\text { Apocynaceous: } 16,17,19,20 \text {-tetrahydro-2,16- } \\
\text { dehydro-18-deoxy isostrychnine, "alkaloid } \\
\text { 376", 10-hydroxyakuammidine, vincanol, } \\
\text { deoxyvincamine, (-)-eburnamenine, } \\
\text { stemmadenine- } N(4) \text {-oxide, strictosamide, 9-( } \beta \text { - } \\
\text { D-gluocopranosyloxy)tetrahydro alstonine; } \\
\text { others: } N \text {-methyl-carbazole }\end{array}$ & Liu et al. (2016) \\
\hline Psychotria psychotriifolia & n.d & - Others: iridoids, polyphenols & Berger (2012) \\
\hline Psychotria pisonioides & n.d & - Others: polyphenols & $\begin{array}{l}\text { Berger }(2012, \text { misidentified as } P s y . \\
\text { convergens) }\end{array}$ \\
\hline \multirow[t]{2}{*}{ Psychotria prainii } & n.d & - Others: carbamates, iridoids & Tran et al. (2019) \\
\hline & n.d & $\begin{array}{l}\text { Others: coumarins, flavonoids, polyphenols, } \\
\text { megastigmanes, triterpenes }\end{array}$ & Yang et al. (2018) \\
\hline \multirow[t]{2}{*}{ Psychotria punctata } & n.s & - Others: polyphenols & $\begin{array}{l}\text { Berger (2012, as P. kirkii); Schindler } \\
\text { et al. (2021) }\end{array}$ \\
\hline & n.s. ${ }^{3}$ & - Others: polyamines & Van Elst et al. (2013 (as Psy. kirkii) \\
\hline
\end{tabular}


Table 11 continued

\begin{tabular}{|c|c|c|c|}
\hline Accepted species & Alkaloids & Compounds and/or compound groups & References (synonyms) \\
\hline & n.s. ${ }^{3}$ & - Others: aminocyclitols & Sieber et al. (2015 (as Psy. kirkii) \\
\hline \multirow[t]{5}{*}{ Psychotria serpens } & n.s & - Others: iridoids & Inouye et al. (1988) \\
\hline & n.d & - Others: triterpenes & Lee et al. (1988) \\
\hline & n.d & - Others: polyphenols & Berger (2012) \\
\hline & n.d & - Others: flavonoids & Lin et al. (2015) \\
\hline & n.d & - Others: flavonoids, triterpenes & Zhou et al. (2018) \\
\hline Psychotria subsessilis & n.d & - Others: iridoids, polyphenols & Berger (2012, as $P$. quinqueradiata) \\
\hline Psychotria sylvivaga & n.d & - Others: iridoids, polyphenols & Berger (2012) \\
\hline Psychotria tenuifolia & n.d & - Others: polyphenols & Berger (2012) \\
\hline Psychotria viridiflora & n.s. ${ }^{3}$ & - Others: polyamines & Van Elst et al. (2013) \\
\hline \multirow[t]{5}{*}{ Psychotria viridis } & IA, $\beta$ CA & $\begin{array}{l}\text { - } N \text {-Methyltryptamine, } N, N \text {-dimethyltryptamine, } \\
\text { 2-methyl tetrahydro- } \beta \text {-carboline }\end{array}$ & Rivier and Lindgren (1972) \\
\hline & IA & - N,N-Dimethyltryptamine & Blackledge and Taylor (2003) \\
\hline & IA & - $N, N$-Dimethyltryptamine & Callaway et al. (2005) \\
\hline & n.s & - Others: polyphenols & Berger (2012) \\
\hline & IA & $\begin{array}{l}\text { - } N \text {-Methyltryptamine, } N, N \text {-dimethyltryptamine; } \\
\text { others: triterpenes, hydrocarbons, fatty acids, } \\
\text { triglycerides }\end{array}$ & Soares et al. (2017) \\
\hline Psychotria yunnanensis & n.d & $\begin{array}{l}\text { - Others: lignans, monoterpenes, megastigmanes, } \\
\text { polyphenols, sesquiterpenes }\end{array}$ & Lu et al. (2014b, 2014c) \\
\hline Psychotria zeylanica & n.d & - Others: iridoids, polyphenols & Berger (2012) \\
\hline Psychotria sp. & n.d & - Others: polyphenols, sphingolipids, triterpenes & $\begin{array}{l}\text { Ye et al. (2014); Zhang et al. } \\
\quad(2010,2012,2013)\end{array}$ \\
\hline Psychotria sp. ${ }^{5}$ & n.d & - Others: megastigmanes & $\begin{array}{l}\text { Tan et al. (2014, misidentified as Psy. } \\
\text { cadingensis) }\end{array}$ \\
\hline identification unclear $^{6}$ & n.d & - Others: triterpenes & $\begin{array}{l}\text { Sandra et al. } 2018 \text { (misidentified as } \\
\text { Pal. croceoides) }\end{array}$ \\
\hline
\end{tabular}

All individual alkaloids are listed and their corresponding alkaloid groups are noted. For further detected compounds ("others") only the respective structural and/or biosynthetic groups are mentioned. Most studies were reported from names now considered homooder heterotypic synonyms, and these names are given in brackets after the corresponding references. Note that phytochemical studies are often tailored to certain compound groups and do not necessarily reflect the full phytochemical complement of a studied species. PA: "protoalkaloids"; TIQA: tetrahydroisoquinoline alkaloids; TA: tryptamine analogues; PIA: polypyrroloindoline alkaloids; $\beta$ CA: $\beta$-carboline alkaloids; MIA: monoterpene-indole alkaloids; n.s.: no alkaloids studied; n.d.: no alkaloids detected

${ }^{1}$ The study was limited to the detection and quantification of flavonoids

${ }^{2} \mathrm{~A}$ nomenclatural combination transferring the species to the genus it belongs according to morphological and/or molecular data is not yet available (comb. ined.)

${ }^{3}$ The studies were limited to detecting polyamines and aminocyclitols

${ }^{4}$ Probably confused with a species of Eumachia, see comments in Taylor et al. (2017: 316)

${ }^{5}$ Psychotria cadigensis, now thought to be extinct, was a narrow endemic to Mt. Cadig, Luzon Island (Philippines; Sohmer and Davis, 2007). The report of this species from Mindoro Island is likely based on a misidentification

${ }^{6}$ Sandra et al. (2018) report the isolation of a triterpene from Nigerian material of 'Palicourea croceoides', but the genus is endemic to the Neotropics. No voucher specimen was cited and the authors did not respond to numerous requests. Although they indeed include a photography of Palicourea croceoides, it cannot serve as voucher on the identity of the species because it was actually taken on the Caribean Island of Dominica in 2006 and copy-pasted without indication of source. Cal.: Calycodendron; Ceph.: Cephaelis; Geo.: Geophila; Hod.: Hodgkinsonia; Marg.: Margaritopsis; Pal.: Palicourea; Psy: Psychotria 


\section{References}

Abdullah NS, Ahmad WYW, Sabri NA (2017a) The chemical constituents from young tubers of Hydnophytum formicarum. Malaysian J Anal Sci 21(2):291-297. https://doi. org/10.17576/mjas-2017-2102-03

Abdullah NS, Ahmad WYW, Sabri NA (2017b) New compounds from Hydnophytum formicarum young tubers. Malaysian J Anal Sci 21(4):778-783. https://doi.org/10. 17576/mjas-2017-2104-03

Achenbach H, Lottes M, Waibel R, Karikas GA, Correa MD, Gupta MP (1995) Alkaloids and other compounds from Psychotria correae. Phytochemistry 38(6):1537-1545. https://doi.org/10.1016/0031-9422(94)00823-C

Achenbach H, Benirschke M, Torrenegra R (1997) Alkaloids and other compounds from seeds of Tabernaemontana cymosa. Phytochemistry 45(2):325-335. https://doi.org/ 10.1016/S0031-9422(96)00645-0

Adjibadé Y, Kuballa B, Cabalion P, Anton R (1986) A new alkaloid from Psychotria forsteriana. Planta Med 52(6):523. https://doi.org/10.1055/s-2007-969305

Adjibadé Y, Kuballa B, Cabalion P, Anton R (1989) Preliminary chemical study of the alkaloids from the fruits of Psychotria forsteriana. Planta Med 55(1):115. https://doi.org/10. 1055/s-2006-961898

Adjibadé Y, Saad H, Sévenet T, Kuballa B, Quirion JC, Anton R (1990) New polyindolenine alkaloids from Calycodendron milnei. Planta Med 56(2):212-215. https://doi.org/10. 1055/s-2006-960927

Adjibadé Y, Weniger B, Quirion JC, Kuballa B, Cabalion P, Anton R (1992) Dimeric alkaloids from Psychotria forsteriana. Phytochemistry 31(1):317-319. https://doi. org/10.1016/0031-9422(91)83062-P

Adjibadé Y, Kuballa B, Cabalion P, Anton R (1985) Occurring of calycanthine: a bis-tetrahydroquinoline alkaloid in the stem-bark of Psychotria forsteriana A. Gray (Rubiaceae). Acta Agron Hung 34(suppl.):81

Aimi N, Tsuyuki T, Murakami H, Sakai SI, Haginiwa J (1985) Structure of ophiorines A and B; novel type gluco indole alkaloids isolated from Ophiorrhiza spp. Tetrahedron Lett 26(43):5299-5302. https://doi.org/10.1016/S00404039(00)95021-4

Akinboye ES, Rosen MD, Bakare O, Denmeade SR (2017) Anticancer activities of emetine prodrugs that are proteolytically activated by the prostate specific antigen (PSA) and evaluation of in vivo toxicity of emetine derivatives. Bioorg Med Chem 25(24):6707-6717. https://doi.org/10. 1016/j.bmc.2017.11.015

Alibasyah ZM, Purba A, Setiabudiawan B, Adhita HD, Kurnia D, Satari MH (2017) The effectiveness of flavonoids and terpenoid isolate Sarang Semut (Myrmecodia pendens Merr and Perry) against Phorphyromonas gingivalis ATCC 33277. Int J Dev Res 7(7):13557-13561

Allen JR, Holmstedt BR (1980) The simple $\beta$-carboline alkaloids. Phytochemistry 19(8):1573-1582. https://doi.org/10. 1016/S0031-9422(00)83773-5

Alves VG, da Rosa EA, de Arruda LLM, Rocha BA, Bersani Amado CA, Santin SMO, Pomini AM, da Silva CC (2016) Acute toxicity, antiedematogenic activity, and chemical constituents of Palicourea rigida Kunth. Z Naturforsch C:
Biosci 71(3-4):39-43. https://doi.org/10.1515/znc-20150036

Alves VG, Schuquel ITA, Ferreira HD, Santin SMO, da Silva CC (2017) Coumarins from roots of Palicourea rigida. Chem Nat Comp 53(6):1157-1159. https://doi.org/10. 1007/s10600-017-2224-8

Amador TA, Elisabetsky E, de Souza DO (1996) Effects of Psychotria colorata alkaloids in brain opioid system. Neur Res 21(1):97-102. https://doi.org/10.1007/BF02527677

Amador TA, Verotta L, Nunes DS, Elisabetsky E (2000) Antinociceptive profile of hodgkinsine. Planta Med 66(8):770-772. https://doi.org/10.1055/s-2000-9604

Andersson L (2002) Re-establishment of Carapichea (Rubiaceae, Psychotrieae). Kew Bull 57(2):363-374. https://doi. org/10.2307/4111112

Andrade JMM, Biegelmeyer R, Xavier CAG, Bordignon SAL, Moreno PRH, Zuanazzi JAS, Henriques AT, Apel MA (2010) Essential oil constituents of Psychotria leiocarpa. Chem Nat Compd 46(4):649-650. https://doi.org/10.1007/ s10600-010-9702-6

Anet EFLJ, Hughes GK, Ritchie E (1961) Hodgkinsine, the alkaloid of Hodgkinsonia frutescens F. Muell Austr J Chem 14(1):173-174. https://doi.org/10.1071/CH9610173

Aniszewski T (2015) Alkaloids: Chemistry, Biology, Ecology, and Applications, 2nd edn. Elsevier, Amsterdam

Barleben L, Panjikar S, Ruppert M, Koepke J, Stöckigt J (2007) Molecular architecture of strictosidine glucosidase: the gateway to the biosynthesis of the monoterpenoid indole alkaloid family. Plant Cell 19(9):2886-2897. https://doi. org/10.1105/tpc.106.045682

Barrabé L, Davis AP (2013) (2207) Proposal to conserve the name Margaritopsis against Eumachia (Rubiaceae. Taxon 62(5):1069-1070

Barrabé L, Buerki S, Mouly A, Davis AP, Munzinger J, Maggia L (2012) Delimitation of the genus Margaritopsis (Rubiaceae) in the Asian, Australasian and Pacific region, based on molecular phylogenetic inference and morphology. Taxon 61(6):1251-1268. https://doi.org/10.1002/tax. 616007

Barrabé L, Maggia L, Pillon Y, Rigault F, Mouly A, Davis AP, Buerki S (2014) New Caledonian lineages of Psychotria (Rubiaceae) reveal different evolutionary histories and the largest documented plant radiation for the archipelago. Mol Phylogenet Evol 71:15-35. https://doi.org/10.1016/j. ympev.2013.10.020

Battersby AR, Harper BJT (1959) 347. Ipecacuanha alkaloids. Part II. The structure of protoemetine and a partial synthesis of (-)-emetine. J Chem Soc 1959:1748-1753. https:// doi.org/10.1039/JR9590001748

Battersby AR, Davidson GC, Harper BJT (1959) 346. Ipecacuanha alkaloids. Part I. Fractionation studies and the isolation of two new alkaloids. $J$ Chem Soc 1959:1744-1748. https://doi.org/10.1039/JR9590001744

Benevides PJC, Young MCM, da Silva BV (2005) Biological Activities of Constituents from Psychotria spectabilis. Pharm Biol 42(8):565-569. https://doi.org/10.1080/ 13880200490901780

Berger A (2017) Two new combinations, lectotypifications and a new name for Costa Rican Palicourea s.l. PhytoKeys 80:53-63. https://doi.org/10.3897/phytokeys.80.13330 
Berger A, Fasshuber H, Schinnerl J, Robien W, Brecker L, Valant-Vetschera K (2011) Iridoids as chemical markers of false ipecac (Ronabea emetica), a previously confused medicinal plant. J Ethnopharmacol 138(3):756-761. https://doi.org/10.1016/j.jep.2011.10.024

Berger A, Fasshuber H, Schinnerl J, Brecker L, Greger H (2012) Various types of tryptamine-iridoid alkaloids from Palicourea acuminata (=Psychotria acuminata Rubiaceae). Phytochemistry Lett 5(3):558-562. https://doi.org/10. 1016/j.phytol.2012.05.013

Berger A, Kostyan MK, Klose SI, Gastegger M, Lorbeer E, Brecker L, Schinnerl J (2015) Loganin and secologanin derived tryptamine-iridoid alkaloids from Palicourea crocea and $P$. padifolia (Rubiaceae). Phytochemistry 116:162-169. https://doi.org/10.1016/j.phytochem.2015. 05.013

Berger A, Preinfalk A, Windberger M, Fasshuber HK, Gastegger M, Klose I, Robien W, Felsinger S, Brecker L, Valant-Vetschera K, Schinnerl J (2016) New reports on flavonoids, benzoic- and chlorogenic acids as rare features in the Psychotria alliance (Rubiaceae). Biochem Syst Ecol 66:145-153. https://doi.org/10.1016/j.bse.2016.02.027

Berger A, Tanuhadi E, Brecker L, Schinnerl J, Valant-Vetschera K (2017) Chemodiversity of tryptamine-derived alkaloids in six Costa Rican Palicourea species (Rubiaceae-Palicoureeae). Phytochemistry 143:124-131. https://doi.org/ 10.1016/j.phytochem.2017.07.016

Berger A, Schinnerl J (2019) Taxonomical and phytochemical diversity of Costa Rica Palicoureeae and Psychotrieae (Rubiaceae). Acta ZooBot Austria 156:231-248. https:// www.zobodat.at/pdf/VZBG_156_0231-0248.pdf

Berger A, Valant-Vetschera K, Schinnerl J, Brecker L (2021) Alkaloid diversification in the genus Palicourea (Rubiaceae: Palicoureeae) viewed from a (retro-)biosynthetic perspective. Phytochem Rev. https://doi.org/10.1007/ s11101-021-09768-y

Berger A (2012) Distribution and systematic significance of selected secondary metabolites within Psychotrieae/Palicoureeae (Rubiaceae). Diploma thesis, University of Vienna. https://doi.org/10.25365/thesis.24493

Berger A (2018a) Rediscovery of Chamisso's type specimens of Hawaiian Psychotria (Rubiaceae, Psychotrieae) in the herbarium of the Natural History Museum, Vienna. PhytoKeys 114:27-42. https://doi.org/10.3897/phytokeys.114. 29426

Berger A (2018b) Synopsis and typification of Mexican and Central American Palicourea, part I: The entomophilous species. Ann. Naturhist. Mus. Wien, Ser. B 120:59-140. http://www.jstor.org/stable/26335282

Bernhard M, Fasshuber H, Robien W, Brecker L, Greger H (2011) Dopamine-iridoid alkaloids in Carapichea affinis (= Psychotria borucana) confirm close relationship to the vomiting root Ipecac. Biochem Syst Ecol 39(3):232-235. https://doi.org/10.1016/j.bse.2011.03.006

Bertelli PR, Biegelmeyer R, Rico EP, Klein-Junior LC, Toson NSB, Minetto L, Bordignon SAL, Gasper AL, Moura S, de Oliveira DL, Henriques AT (2017) Toxicological profile and acetylcholinesterase inhibitory potential of Palicourea deflexa, a source of $\beta$-carboline alkaloids. Comp Biochem Physiol Part C 201:44-50. https://doi.org/10.1016/j.cbpc. 2017.09.003
Bertelli PR, Rico EP, Grünspan LD, Biegelmeyer R, KleinJúnior LC, Vander Heyden Y, Gasper AL, Bordignon SAL, Oliveira DL, Henriques AT (2015) Application of zebrafish embryos toxicity test to evaluate the alkaloid fraction of Psychotria deflexa. Planta Med 81(16):PM_160. https:// doi.org/10.1055/s-0035-1565537

Blackledge RD, Taylor CM (2003) Psychotria viridis -A botanical source of dimethyltryptamine (DMT). Microgram 1(1-2):18-22

Bolzani VS, Trevisan LMV, Young MCM (1992) Triterpenes of Palicourea rigida H.B.K. Rev Latinoam Quim 23(1):20-21

Borhidi AL (2011) Transfer of the Mexican species of Psychotria subgen. Heteropsychotria to Palicourea based on morphological and molecular evidences. Acta Bot Hung 53(3-4):241-250. https://doi.org/10.1556/abot.53.2011.34.4

Borhidi AL (2017) La circunscripsión de Palicourea subgen. Heteropsychotria (Rubiaceae, Palicoureeae). Acta Bot Hung 59(1-2):25-61. https://doi.org/10.1556/034.59. 2017.1-2.4

Both FL, Kerber VA, Henriques AT, Elisabetsky E (2002) Analgesic properties of umbellatine from Psychotria umbellata. Pharm Biol 40(5):336-341. https://doi.org/10. 1076/phbi.40.5.336.8453

Brand G, Henriques AT, Passos CDS, Baldoqui DC, de Oliveira Santin SM, da Costa WF, Sarragiotto MH (2012) Pyrrolidinoindoline alkaloids from Margaritopsis cymuligera (Muell. Arg.) C.M. Taylor (Rubiaceae). Biochem Syst Ecol 45:155-157. https://doi.org/10.1016/j.bse.2012.07.009

Bruniera CP (2015) Sistemática e taxonomia de Rudgea Salisb. (Palicoureeae, Rubiaceae). Doctoral Thesis, Universidade de São Paulo. https://doi.org/10.11606/T.41.2015.tde28072015-145432

Cai X-H, Bao M-F, Zhang Y, Zeng C-X, Liu Y-P, Luo X-D (2011) A new type of monoterpenoid indole alkaloid precursor from Alstonia rostrata. Org Lett 13(14):3568-3571. https://doi.org/10.1021/ol200996a

Calixto NO, Pinto MEF, Ramalho SD, Burger M, Bobey AF, Young MCM, Bolzani VS, Pinto AC (2016) The genus Psychotria: Phytochemistry, chemotaxonomy, ethnopharmacology and biological properties. J Braz Chem Soc 27(8):1355-1378. https://doi.org/10.5935/0103-5053. 20160149

Calixto NO, Cordeiro MS, Giorno TBS, Oliveira GG, Lopes NP, Fernandes PD, Pinto AC, Rezende CM (2017) Chemical constituents of Psychotria nemorosa Gardner and antinociceptive activity. J Braz Chem Soc 28(5):707-723. https://doi.org/10.21577/0103-5053.20160219

Callaway JC, Brito GS, Neves ES (2005) Phytochemical analyses of Banisteriopsis caapi and Psychotria viridis. J Psych Drugs 37(2):145-150. https://doi.org/10.1080/02791072. 2005.10399795

Canham SM, Hafensteiner BD, Lebsack AD, May-Dracka TL, Nam S, Stearns BA, Overman LE (2015) Stereocontrolled enantioselective total synthesis of the $[2+2]$ quadrigemine alkaloids. Tetrahedron 71(37):6424-6436. https://doi.org/ 10.1016/j.tet.2015.02.080

Chen T, Taylor CM (2011) Psychotria. In: Yang QE, Landrein S, Osborne J, Borosova R (eds) Flora of China, vol 19. 
Science Press and Missouri Botanical Garden, Beijing and St, Louis, pp 294-301

Coelho EG, Amaral ACF, Ferreira JLP, dos Santos AG, Pinheiro MLB, de Silva AJR (2007) Calcium oxalate crystals and methyl salicylate as toxic principles of the fresh leaves from Palicourea longiflora, an endemic species in the Amazonas state. Toxicon 49(3):407-409. https://doi.org/ 10.1016/j.toxicon.2006.10.003

Cook D, Lee ST, Taylor CM, Bassüner B, Riet-Correa F, Pfister JA, Gardner DR (2014) Detection of toxic monofluoroacetate in Palicourea species. Toxicon 80:9-16. https://doi. org/10.1016/j.toxicon.2013.12.003

Cordell GA, Quinn-Beattie ML, Farnsworth NR (2001) The potential of alkaloids in drug discovery. Phytother Res 15(3):183-205. https://doi.org/10.1002/ptr.890

da Cunha ALMC, Sá A, Mello SC, Vásquez-Castro YE, Luna AS, Aucelio RQ (2016) Determination of nitrogen-containing polycyclic aromatic compounds in diesel and gas oil by reverse-phase high performance liquid chromatography using introduction of sample as detergentless microemulsion. Fuel 176:119-129. https://doi.org/10. 1016/j.fuel.2016.02.035

de Carvalho Junior AR, Oliveira Ferreira R, de Souza Passos M, da Silva Boeno SI, de Lima Glória das Virgens L, Ventura TLB, Calixto SD, Lassounskaia E, de Carvalho MG, BrazFilho R, Curcino Vieira IJ (2019) Antimycobacterial and nitric oxide production inhibitory activities of triterpenes and alkaloids from Psychotria nuda (Cham. \& Schltdl.) Wawra. Molecules 24(6):1026. https://doi.org/10.3390/ molecules 24061026

de Carvalho Junior AR, Ferreira RO, de Souza Passos M, Vieira MGC, de Lima Glória das Virgens L, Calixto SD, Ventura TLBV, Lassounskaia E, de Carvalho MG, Braz-Filho R, Vieira IJC (2021) Chemical composition, antimycobacterial and anti-inflammatory activities of iridoids and triterpene from Psychotria suterella (Rubiaceae). Pharmacogn Mag 17(74):355-359. https://doi.org/10.4103/pm.pm_93_ 2

de Freitas Morel LJ, Baratto DM, Pereira PS, Contini SHT, Momm HG, Bertoni BW, de Castro S (2011) Loganin production in Palicourea rigida HBK (Rubiaceae) from populations native to Brazilian Cerrado. J Med Plants Res 5(12):2559-2565. https://doi.org/10.5897/JMPR.9000856

de Sousa Queiroz C, de Carvalho Batista FR, de Oliveira LO (2011) Evolution of the 5.8 S nrDNA gene and internal transcribed spacers in Carapichea ipecacuanha (Rubiaceae) within a phylogeographic context. Mol Phylogenetics Evol 59(2):293-302. https://doi.org/10.1016/j. ympev.2011.01.013

da Rosa EA, Silva BC, Silva FM, Tanaka C, Peralta RM, de Oliveira C, Kato L, Ferreira HD, da Silva CC (2010) Flavonoids and antioxidant activity in Palicourea rigida Kunth Rubiaceae. Rev Bras Farmacogn 20(4):484-488. https://doi.org/10.1590/S0102-695X2010000400004

da Silva VC, de Carvalho MG, Alves AN (2008) Chemical constituents from leaves of Palicourea coriacea (Rubiaceae). J Nat Med 62(3):356-357. https://doi.org/10.1007/ s11418-008-0227-2

da Silva MDS, Pereira AMS, de Freitas Morel LJ, de Castro FS, Bertoni BW (2013) Association of loganin contents with the genetic characterization of natural populations of
Palicourea rigida Kunth determined by AFLP molecular markers. Biochem Syst Ecol 51:189-194. https://doi.org/ 10.1016/j.bse.2013.08.032

Dan S, Dan SS (1986) Phytochemical study of Adansonia digitata, Coccoloba excoriata Psychotria Adenophylla and Schleichera Oleosa. Fitoterapia 57(6):445-446

Dash UC, Kanhar S, Dixit A, Dandapat J, Sahoo AK (2019) Isolation, identification, and quantification of pentylcurcumene from Geophila repens: A new class of cholinesterase inhibitor for Alzheimer's disease. Bioorg Chem 88:102947. https://doi.org/10.1016/j.bioorg.2019.102947

de Cacia OM, Negri G, Salatino A, Braga MR (2007) Detection of anthraquinones and identification of 1,4-naphtohydroquinone in cell suspension cultures of Rudgea jasminoides (Rubiaceae). Rev Bras Biol 30(1):167-172. https://doi.org/ 10.1590/S0100-84042007000100017

de Carvalho Junior AR, Vieira IJC, de Carvalho MG, Braz-Filho R, Lima MAS, Ferreira RO, Maria JE, de Oliveira DB (2017) ${ }^{13}$ C-NMR Spectral data of alkaloids isolated from Psychotria species (Rubiaceae). Molecules 22(1):103. https://doi.org/10.3390/molecules22010103

de Oliveira AM, Lemos RPL, Conserva LM (2013) $\beta$-Carboline alkaloids from Psychotria barbiflora DC. (Rubiaceae). Biochem Syst Ecol 50:339-341. https://doi.org/10.1016/j. bse.2013.04.015

de L Carvalho FK, Cook D, Lee ST, Taylor CM, Oliveira JBS, Riet-Correa F (2016) Determination of toxicity in rabbits and corresponding detection of monofluoroacetate in four Palicourea (Rubiaceae) species from the Amazonas state, Brazil. Toxicon 109:42-44. https://doi.org/10.1016/j. toxicon.2015.11.009

Delprete PG, Kirkbride Jr. JH (2015) New combinations in Eumachia (Rubiaceae) for species occurring on the Guiana Shield. J Bot Res Inst Texas 9(1):75-79. https://www.jstor. org/stable/24621246

Delprete PG, Kirkbride Jr. JH (2016) New combinations and new names in Palicourea (Rubiaceae) for species of Psychotria subgenus Heteropsychotria occurring in the Guianas. J Bot Res Inst Texas 10(2):409-442. https://www. jstor.org/stable/44858580

Delprete PG, Lachenaud O (2018) Conspectus of Palicourea section Potaroenses (Rubiaceae), with a new species from French Guiana and a new combination. Plant Ecol Evol 151(1):119-129. https://doi.org/10.5091/plecevo.2018. 1356

do Nascimento CA, Gomes MS, Liao LM, de Oliveira C, Kato L, da Silva CC, Tanaka C (2006) Alkaloids from Palicourea coriacea (Cham.) K. Schum. Z Naturforsch B: Chem Sci 61(11):1443-1446. https://doi.org/10.1515/znb2006-1120

do Nascimento CA, Liao LM, Kato L, da Silva CC, Tanaka CM, Schuquel IT, de Oliveira CM (2008) A tetrahydro $\beta$-carboline trisaccharide from Palicourea coriacea (Cham.) K. Schum. Carbohydr Res 343(6):1104-1107. https://doi.org/ 10.1016/j.carres.2008.01.032

dos Santos PC, Simões-Pires CA, Nurisso A, Soldi TC, Kato L, de Oliveira CMA, de Faria EO, Marcourt L, Gottfried C, Carrupt P-A, Henriques AT (2013a) Indole alkaloids of Psychotria as multifunctional cholinesterases and monoamine oxidases inhibitors. Phytochemistry 86:8-20. https://doi.org/10.1016/j.phytochem.2012.11.015 
dos Santos PC, Soldi TC, Torres Abib R, Anders Apel M, Simões-Pires C, Marcourt L, Gottfried C, Henriques AT (2013b) Monoamine oxidase inhibition by monoterpene indole alkaloids and fractions obtained from Psychotria suterella and Psychotria laciniata. J Enzyme Inhib Med Chem 28(3):611-618. https://doi.org/10.3109/14756366. 2012.666536

Düsman LT, Marinho Jorge TC, de Souza MC, Eberlin MN, Meurer EC, Bocca CC, Basso EA, Sarragiotto MH (2004) Monoterpene indole alkaloids from Palicourea crocea. J Nat Prod 67(11):1886-1888. https://doi.org/10.1021/ np0340807

Elisabetsky E, Amador TA, Albuquerque RR, Nunes DS, do CT Carvalho A (1995) Analgesic activity of Psychotria colorata (Willd. ex R. \& S.) Muell. Arg. alkaloids. J Ethnopharmacol 48(2):77-83. https://doi.org/10.1016/ 0378-8741(95)01287-N

El-Seedi HR (1999) Coumarins, benzoic acids and terpenoids from Palicourea demissa. Rev Latinoam Quim 27(1):13-16

Engida AM, Kasim NS, Tsigie YA, Ismadji S, Huynh LH, Ju YH (2013) Extraction, identification and quantitative HPLC analysis of flavonoids from sarang semut (Myrmecodia pendan). Ind Crops Prod 41:392-396. https://doi.org/ 10.1016/j.indcrop.2012.04.043

Engida AM, Faika S, Nguyen-Thi BT, Ju Y-H (2015) Analysis of major antioxidants from extracts of Myrmecodia pendans by UV/visible spectrophotometer, liquid chromatography/tandem mass spectrometry, and high-performance liquid chromatography/UV techniques. J Food Drug Anal 23(2):303-309. https://doi.org/10.1016/j.jfda.2014.07.005

Faria EO, Kato L, Oliveira CMA, Carvalho BG, Silva CC, Sales L, Schuquel IT, Silva-Lacerda EP, Delprete GP (2010) Quaternary $\beta$-carboline alkaloids from Psychotria prunifolia (Kunth) Steyerm. Phytochem Lett 3(3):113-116. https://doi.org/10.1016/j.phytol.2010.02.008

Farias FM, Konrath EL, Zuanazzi JAS, Henriques AT (2008) Strictosamide from Psychotria nuda (Cham. et Schltdl) Wawra (Rubiaceae). Biochem Syst Ecol 36(12):919-920. https://doi.org/10.1016/j.bse.2008.10.002

Farias FM, Passos CS, Arbo MD, Barros DM, Gottfried C, Steffen VM, Henriques AT (2012) Strictosidinic acid, isolated from Psychotria myriantha Müll. Arg. (Rubiaceae), decreases serotonin levels in rat hippocampus. Fitoterapia 83(6):1138-1143. https://doi.org/10.1016/j. fitote.2012.04.013

Fridrichsons J, Mackay MF, Mathieson AM (1967) The molecular structure of hodgkinsine, $\mathrm{C}_{33} \mathrm{H}_{38} \mathrm{~N}_{6}$. Tetrahedron Lett 8(36):3521-3524. https://doi.org/10.1016/ S0040-4039(01)89834-8

Fridrichsons J, Mackay MF, Mathieson AM (1974) The absolute molecular structure of hodgkinsine. Tetrahedron 30(1):85-92. https://doi.org/10.1016/S00404020(01)97221-7

Fu Y-H, Huang L-G, Wang X-C, Li X-B, Li K-K, Wu S-L, Liu Y-P (2015) Studies on chemical constituents from Psychotria straminea. Zhongguo Zhongyao Zazhi [Journal of Chinese Materia Medica] 40(11):2138-2143

Garcia RMA, de Oliveira LO, Moreira MA, Barros WS (2005) Variation in emetine and cephaeline contents in roots of wild Ipecac (Psychotria ipecacuanha). Biochem Syst Ecol 33(3):233-243. https://doi.org/10.1016/j.bse.2004.08.005

Garcia LA de, Tobón CF, Mora CE (1997) Citotoxicidad de los componentes de Palicourea ovalis. Rev Colomb Cienc Quim Farm 26(1):55-57. https://doi.org/10.15446/ rcciquifa

Gartika M, Pramesti HT, Kurnia D, Satari MH (2018) A terpenoid isolated from sarang semut (Myrmecodia pendans) bulb and its potential for the inhibition and eradication of Streptococcus mutans biofilm. BMC Complement Altern Med 18(1):151. https://doi.org/10.1186/s12906-018-2213$\mathrm{x}$

Gentry AH (1990) Floristic similarities and differences between southern Central America and Central Amazonia. In: Gentry AH (ed) Four Neotropical Rainforests. Yale University Press, New Haven, CT, pp 141-157

Giang PM, Son HV, Son PT (2007) Study on the chemistry and antimicrobial activity of Psychotria reevesii Wall. (Rubiaceae). Vietnam J Chem 45(5):628-633. https://doi.org/10. $15625 / 4802$

Gonzalez J, Dieck T (1996) Asperuloside, an iridoid glucoside isolated from Psychotria mariniana. Rev Latinoam Quim 24(1):7-9

Guéritte-Voegelein F, Sévenet T, Pusset J, Adeline MT, Gillet B, Beloeil JC, Guénard D, Potier P, Rasolonjanahary R, Kordon C (1992) Alkaloids from Psychotria oleoides with activity on growth hormone release. J Nat Prod 55(7):923-930. https://doi.org/10.1021/np50085a012

Hadi S, Bremner JB (2001) Initial studies on alkaloids from Lombok medicinal plants. Molecules 6:117-129. https:// doi.org/10.3390/60100117

Hadi S, Rahmawati KP, Asnawati D, Ersalena VF, Azwari A (2014) Characterization of alkaloids from the leaves of Psychotria malayana Jack of Lombok Island on the basis of gas chromatography-mass spectroscopy. J Pure Appl Chem Res 3(3):108-113

Hanh NP, Phan NHT, Thuan NTD, Hanh TTH, Vien LT, Thao NP, Thanh NV, Cuong NX, Binh NQ, Nam NH, Kiem PV, Kim YH, Minh CV (2016) Two new simple iridoids from the ant-plant Myrmecodia tuberosa and their antimicrobial effects. Nat Prod Res 30(18):2071-2076. https://doi.org/ 10.1080/14786419.2015.1113412

Hart NK, Johns SR, Lamberton JA, Summons RE (1974) Psychotridine, a $\mathrm{C}_{55} \mathrm{H}_{62} \mathrm{~N}_{10}$ alkaloid from Psychotria beccarioides (Rubiaceae). Austr J Chem 27(3):639-646

Hasmah A, Hohmann J, Azimahtol HL, Molnar J, Forgo P (2008) Antiproliferative compounds from Hydnophytum formicarium. J Trop Med Plants 9(2):366-371

Hatfield GM, Arteaga L, Dwyer JD, Arias TD, Gupta MP (1981) An investigation of Panamanian Ipecac: botanical source and alkaloid analysis. J Nat Prod 44(4):452-456

Hayashi T, Smith FT, Lee KH (1987) Antitumor agents. 89. Psychorubrin, a new cytotoxic naphthoquinone from Psychotria rubra and its structure-activity relationships. J Med Chem 30(11):2005-2008. https://doi.org/10.1021/ jm00394a013

Henriques AT, Lopes SO, Paranhos JT, Gregianini TS, FettNeto AG, Schripsema J, von Poser GL (2004) N, $\beta$-dGlucopyranosyl vincosamide, a light regulated indole alkaloid from the shoots of Psychotria leiocarpa. 
Phytochemistry 65(4):449-454. https://doi.org/10.1016/j. phytochem.2003.10.027

Inouye H, Takeda Y, Nishimura H, Kanomi A, Okuda T, Puff C (1988) Chemotaxonomic studies of rubiaceous plants containing iridoid glycosides. Phytochemistry 27(8):2591-2598. https://doi.org/10.1016/00319422(88)87030-4

Itoh A, Tanahashi T, Nagakura N (1989) Neoipecoside and 7-methylneoipecoside, new unusually-cyclized tetrahydroisoquinoline-monoterpene glucosides from Cephaelis ipecacuanha. Chem Pharm Bull 37(4):1137-1139. https:// doi.org/10.1248/cpb.37.1137

Itoh A, Tanahashi T, Nagakura N (1991) Six tetrahydroisoquinoline-monoterpene glucosides from Cephaelis ipecacuanha. Phytochemistry 30(9):3117-3123. https://doi.org/ 10.1016/S0031-9422(00)98265-7

Itoh A, Tanahashi T, Nagakura N, Nayeshiro H (1994) Tetrahydroisoquinoline-monoterpene glucosides from Alangium lamarckii and Cephaelis ipecacuanha. Phytochemistry 36(2):383-387. https://doi.org/10.1016/S00319422(00)97080-8

Itoh A, Ikuta Y, Baba Y, Tanahashi T, Nagakura N (1999) Ipecac alkaloids from Cephaelis acuminata. Phytochemistry 52(6):1169-1176. https://doi.org/10.1016/S00319422(99)00361-1

Itoh A, Baba Y, Tanahashi T, Nagakura N (2002) Tetrahydroisoquinoline-monoterpene glycosides from Cephaelis acuminata. Phytochemistry 59(1):91-97. https://doi.org/ 10.1016/S0031-9422(01)00418-6

Jacobs J, Claessens S, de Kimpe N (2008) First straightforward synthesis of 1-hydroxy-3,4-dihydro-1H-benz $[g]$ isochromene-5,10-dione and structure revision of a bioactive benz $[g]$ isochromene-5,10-dione from Psychotria camponutans. Tetrahedron 64(2):412-418. https://doi.org/10. 1016/j.tet.2007.10.063

Jamison CR, Badillo JJ, Lipshultz JM, Comito RJ, MacMillan DWC (2017) Catalyst-controlled oligomerization for the collective synthesis of polypyrroloindoline natural products. Nat Chem 9(12):1165-1169. https://doi.org/10.1038/ nchem. 2825

Jannic V, Guéritte F, Laprévote O, Serani L, Martin MT, Sévenet T, Potier P (1999) Pyrrolidinoindoline alkaloids from Psychotria oleoides and Psychotria lyciiflora. J Nat Prod 62(6):838-843. https://doi.org/10.1016/j.tet.2007.10. 063

Kafua L, Kritzinger Q, Hussein A (2009) Antifungal activity of Psychotria capensis leaf extracts. S Afr J Bot 75(2):434. https://doi.org/10.1016/j.sajb.2009.02.148

Kato L, de Oliveira CMA, Faria EO, Ribeiro LC, Carvalho BG, da Silva CC, Schuquel ITA, Santin MO, Nakamura CV, Britta EA, Miranda N, Iglesias AH, Delprete PG (2012) Antiprotozoal alkaloids from Psychotria prunifolia (Kunth) Steyerm. J Braz Chem Soc 23(2):355-360. https:// doi.org/10.1590/S0103-50532012000200024

Kato L, Moraes AP, de Oliveira CMA, Vaz BG, de Almeida Gonçalves L, e Silva EC, Janfelt C (2017) The spatial distribution of alkaloids in Psychotria prunifolia (Kunth) Steyerm and Palicourea coriacea (Cham.) K. Schum leaves analysed by desorption electrospray ionisation mass spectrometry imaging. Phytochem Anal 29(1):69-76. https://doi.org/10.1002/pca.2715
Kemmerling W (1996) Toxicity of Palicourea marcgravii: combined effects of fluoroacetate, $N$-methyltyramine and 2-methyltetrahydro- $\beta$-carboline. Z Naturforsch, C: Biosci 51(1-2):59-64. https://doi.org/10.1515/znc-1996-1-211

Kerber VA, Gregianini TS, Paranhos JT, Schwambach J, Farias F, Fett JP, Fett-Neto AG, Zuanazzi JA, Quirion JC, Elizabetsky E, Henriques AT (2001) Brachycerine, a novel monoterpene indole alkaloid from Psychotria brachyceras. J Nat Prod 64(5):677-679. https://doi.org/10.1021/ np000590e

Kerber VA, Passos CS, Verli H, Fett-Neto AG, Quirion JP, Henriques AT (2008) Psychollatine, a glucosidic monoterpene indole alkaloid from Psychotria umbellata. J Nat Prod 71(4):697-700. https://doi.org/10.1021/ np0703951

Kerber VA, Passos CS, Klein-Júnior LC, Quirion J-C, Pannecoucke X, Salliot-Maire I, Henriques AT (2014) Three new monoterpene indole alkaloids from Psychotria umbellata Thonn. Tetrahedron Lett 55(4):4798-4800. https://doi.org/10.1016/j.tetlet.2014.06.090

Kiehn M, Berger A (2020) Neotropical Rubiaceae: Synthesis of chromosome data from Costa Rican taxa, with insights on the systematics of the family. Ann Missouri Bot Gard 105(4):423-458. https://doi.org/10.3417/2020421

Klein-Júnior LC, Cretton S, Allard P-M, Genta-Jouve G, Passos CS, Salton J, Bertelli P, Pupier M, Jeannerat D, Vander Heyden Y, Gasper AL, Wolfender J-L, Christen P, Henriques AT (2017) Targeted isolation of monoterpene indole alkaloids from Palicourea sessilis. J Nat Prod 80(11):3032-3037. https://doi.org/10.1021/acs.jnatprod. $7 \mathrm{~b} 00681$

Koehbach J, Attah AF, Berger A, Hellinger R, Kutchan TM, Carpenter EJ, Rolf M, Sonibare MA, Moody JO, Wong GK, Dessein S, Greger H, Gruber CW (2013) Cyclotide discovery in Gentianales revisited-identification and characterization of cyclic cystine-knot peptides and their phylogenetic distribution in Rubiaceae plants. Biopolymers 100(5):438-452. https://doi.org/10.1002/bip.22328

Kornpointner C, Berger A, Fischer IM, Popl L, Groher C, Valant-Vetschera K, Brecker L, Schinnerl J (2018) Revisiting Costa Rican Carapichea affinis (Rubiaceae: Palicoureeae): A source of bioactive dopamine-iridoid alkaloids. Phytochemistry Lett 26:164-169. https://doi. org/10.1016/j.phytol.2018.05.004

Kornpointner C, Berger A, Traxler F, Habziadic A, Massar M, Matek J, Brecker L, Schinnerl J (2020) Alkaloid and iridoid glucosides from Palicourea luxurians (Rubiaceae: Palicoureeae) indicate tryptamine and tryptophan iridoid alkaloid formation apart the strictosidine pathway. Phytochemistry 173:112296. https://doi.org/10.1016/j. phytochem.2020.112296

Kostyan MK (2017) Comparative analysis of secondary metabolites in selected Notopleura species, Master Thesis, University of Vienna, Vienna, Austria. https://doi.org/10. 25365/thesis.49735

Kurnia D, Apriyanti E, Soraya C, Satari MH (2019) Antibacterial flavonoids against oral bacteria of Enterococcus faecalis ATCC 29212 from Sarang Semut (Myrmecodia pendans) and its inhibitor activity against Enzyme MurA. Curr Drug Discov Technol 16(3):290-296. https://doi.org/ $10.2174 / 1570163815666180828113920$ 
Lachenaud O (2019) Révision du genre Psychotria (Rubiaceae) en Afrique Occidentale et Centrale. Opera Bot Belg 17:1-909

Lajis NH, Mahmud Z, Toia RF (1993) The alkaloids of Psychotria rostrata. Planta Med 59(4):383-384. https://doi. org/10.1055/s-2006-959709

Leal MB, Elisabetsky E (1996) Absence of alkaloids in Psychotria carthagenensis Jacq. (Rubiaceae). J Ethnopharmacol 54(1):37-40. https://doi.org/10.1016/03788741(96)01448-1

Lee MR (2008) Ipecacuanha: the South American vomiting root. J R Coll Physicians Edinb 38(4):355-360

Lee K-H, Lin Y-M, Wu T-S, Zhang D-C, Yamagishi T, Hayashi T, Hall IH, Chang J-J, Wu R-Y, Yang T-H (1988) Antitumor agents. 88. The cytotoxic principles of Prunella vulgaris, Psychotria serpens, and Hyptis capitata: ursolic acid and related derivatives. Planta Med 54(4):308-311. https://doi.org/10.1055/s-2006-962441

Li H-F, Huang J, Liu M-S, Zhang X-P (2011a) Studies on chemical constituents from leaves of Psychotria hainanensis. Zhongguo Shiyan Fangjixue Zazhi 17(19):125-127

Li XN, Zhang Y, Cai XH, Feng T, Liu YP, Li Y, Ren J, Zhu HJ, Luo XD (2011b) Psychotripine: a new trimeric pyrroloindoline derivative from Psychotria pilifera. Org Lett 13(21):5896-5899. https://doi.org/10.1021/ol202536b

Libot F, Miet C, Kunesch N, Poisson JE, Pusset J, Sévenet T (1987) Rubiacées d'Océanie: Alcaloïdes de Psychotria oleoides de Nouvelle-Calédonie et de Calycodendron milnei du Vanuatu (Nouvelles-Hébrides). J Nat Prod 50(3):468-473. https://doi.org/10.1021/np50051a020

Libot F, Kunesch N, Poisson J, Kaiser M, Duddeck H (1988) Biomimetic transformation of hodgkinsine, a pyrrolidinoindoline alkaloid. Heterocycles 27(10):2381-2386. https://doi.org/10.3987/COM-88-4620

Lin CZ, Wu AZ, Zhong Y, Wang YM, Peng GT, Su XJ, Liu BX, Deng Y, Zhu CC, Zhang CX (2015) Flavonoids from Psychotria serpens $\mathrm{L}$, a herbal medicine with anti-cancer activity. J Cancer Res Updates 4(2):60-64. https://doi.org/ 10.6000/1929-2279.2015.04.02.3

Liu Y, Wang J-S, Wang X-B, Kong L-Y (2013) Two novel dimeric indole alkaloids from the leaves and twigs of Psychotria henryi. Fitoterapia 86:178-182. https://doi.org/ 10.1016/j.fitote.2013.03.013

Liu Y, Wang J-S, Wang X-B, Kong L-Y (2014) Absolute configuration study of a new dimeric indole alkaloid from the leaves and twigs of Psychotria henryi. J Asian Nat Prod Res 16(1):29-33. https://doi.org/10.1080/10286020.2013. 870996

Liu L, Song C-W, Khan A, Li X-N, Yang X-W, Cheng G-G, Liu Y-P, Luo X-D (2016) A potent antibacterial indole alkaloid from Psychotria pilifera. J Asian Nat Prod Res 18(8):798-803. https://doi.org/10.1080/10286020.2016. 1158710

Lopes MN, Mazza FC, Young MCM, da S Bolzani V, (1999) Complete assignments of $1 \mathrm{H}$ and 13C-NMR spectra of the 3,4-seco-triterpene canaric acid isolated from Rudgea jasminoides. J Braz Chem Soc 10(3):237-240. https://doi. org/10.1590/S0103-50531999000300013

Lopes SO, Moreno PRH, Henriques AT (2000) Growth characteristics and chemical analysis of Psychotria carthagenensis cell suspension cultures. Enzyme Microb Tech
26(2):259-264.

https://doi.org/10.1016/S01410229(99)00148-9

Lopes S, von Poser GL, Kerber VA, Farias FM, Konrath EL, Moreno P, Sobral ME, Zuanazzi JA, Henriques AT (2004) Taxonomic significance of alkaloids and iridoid glucosides in the tribe Psychotrieae (Rubiaceae). Biochem Syst Ecol 32(12):1187-1195. https://doi.org/10.1016/j.bse.2004.04. 015

Lopes SO (1998) Análise química e cultivo in vitro de Psychotria leiocarpa Cham. \& Schlecht. e Psychotria carthagenensis Jacq. (Rubiaceae). Master Thesis, Universidade Federal do Rio Grande do Sul

Lorence DH, Taylor CM (2012) Rubiaceae. In: Davidse G, Sousa Sánchez M, Knapp S, Chiang Cabrera F (eds) Flora Mesoamericana, vol 4. Missouri Botanical Garden Press. St. Louis, Missouri, pp 1-288

Lu HX, Liu LY, Li DP, Li JZ, Xu LC (2014a) A new iridoid glycoside from the root of Psychotria rubra. Biochem Syst Ecol 57:133-136. https://doi.org/10.1016/j.bse.2014.07. 024

Lu Q, Wang J, Kong L (2014b) Chemical constituents from Psychotria yunnanensis and its chemotaxonomic study. Biochem Syst Ecol 52:20-22. https://doi.org/10.1016/j. bse.2013.11.002

Lu Q, Wang J, Luo J, Wang X, Shan S, Kong L (2014c) A new acorane sesquiterpene from the aerial parts of Psychotria yunnanensis. Nat Prod Res 28(20):1659-1663. https://doi. org/10.1080/14786419.2014.934234

Luo Y, Li G, Li G, Yan J, Yi J, Zhang G (2011) Discovery and identification of 2-phenylethyl 2,6-dihydroxybenzoate as a natural lipid-lowering lead. Planta Med 77(18):2047-2049. https://doi.org/10.1055/s-0031-1280118

Ma J, Hecht SM (2004) Javaniside, a novel DNA cleavage agent from Alangium javanicum having an unusual oxindole skeleton. Chem Commun 10:1190-1191. https://doi.org/ 10.1039/B402925A

Macoy DM, Kim WY, Lee SY, Kim MG (2015) Biosynthesis, physiology, and functions of hydroxycinnamic acid amides in plants. Plant Biotechnol Rep 9(5):269-278. https://doi. org/10.1007/s11816-015-0368-1

Mahmud Z, Musa M, Ismail N, Lajis NH (1993) Cytotoxic and bacteriocidal activities of Psychotria rostrata. Int $\mathrm{J}$ Pharmacog 31(2):142-146. https://doi.org/10.3109/ 13880209309082931

Martín ML, Gupta MP, Ortiz de Urbina AV, Karikas GA, Gordaliza M, Miguel de Corral JM, San Román L, Sánchez C, San Feliciano A (1994) Pharmacological and phytochemical studies of Cephaelis axillaris. Planta Med 60(6):561-565. https://doi.org/10.1055/s-2006-959572

Martins D, Nunez CV (2015) Secondary metabolites from Rubiaceae species. Molecules 20(7):13422-13495. https:// doi.org/10.3390/molecules200713422

Matsuura HN, Fett-Neto AG (2013) The major indole alkaloid $N, \beta$-d-glucopyranosyl vincosamide from leaves of Psychotria leiocarpa Cham. \& Schltdl. is not an antifeedant but shows broad antioxidant activity. Nat Prod Res 27(4-5):402-411. https://doi.org/10.1080/14786419.2012. 715293

Moreno BP, Fiorucci LLR, do Carmo MRB, Sarragiotto MH, Baldoqui DC, (2014) Terpenoids and a coumarin from aerial parts of Psychotria vellosiana Benth. (Rubiaceae). 
Biochem Syst Ecol 56:80-82. https://doi.org/10.1016/j. bse.2014.04.013

Morita H, Ichihara Y, Takeya K, Watanabe K, Itokawa H, Motidome M (1989) A new indole alkaloid glycoside from the leaves of Palicourea marcgravii. Planta Med 55(3):288-289. https://doi.org/10.1055/s-2006-962007

Moura VM de, Ribeiro MAS, Corrêa JGS, Peixoto MA, Souza GK, Bonfim-Mendonça PS, Svidzinski TIE, Pomini AM, Meurer EC, Santin SMO (2020b) Minutifloroside, a new bis-iridoid glucoside with antifungal and antioxidant activities and other constituents from Palicourea minutiflora. J Braz Chem Soc 31(3):505-511. https://doi.org/10. 21577/0103-5053.20190209

Moura VM. de, Ames FQ, Corrêa JG, Peixoto MA, Amorim AM, Pomini AM, Carvalho JE. de, Ruiz ALTG, BersaniAmado CA, Santin SM (2020a) Cytotoxicity and anti-inflammatory effects of the extract, fractions and alkaloids from Palicourea minutiflora (Rubiaceae). Nat Prod Res. https://doi.org/10.1080/14786419.2019.1710704

Muhammad I, Khan IA, Fischer NH, Fronczek FR (2001) Two stereoisomeric pentacyclic oxindole alkaloids from $U n$ caria tomentosa: uncarine $\mathrm{C}$ and uncarine E. Acta Crystallogr C 57(4):480-482. https://doi.org/10.1107/ S0108270101000932

Muhammad I, Dunbar DC, Khan SI, Tekwani BL, Bedir E, Takamatsu S, Ferreira D, Walker LA (2003) Antiparasitic alkaloids from Psychotria klugii. J Nat Prod 66(7):962-967. https://doi.org/10.1021/np030086k

Müller Argoviensis J (1881) Rubiaceae. Tribus I Retiniphylleae-Tribus VI Psychotrieae. In: von Martius CFP, Eichler AG, Urban I (eds) Flora Brasiliensis: Enumeratio Plantarum in Brasilia 6(5). Apud R. Oldenbourg, Monachii et Lipsiae, pp 1-470. https://www.biodiversitylibrary.org/ item/9666

Murillo R, Castro V (1998) Isolation of the alkaloid harmane from Psychotria suerrensis. Ing Sci Quim 18(2):61-62

Nagakura N, Itoh A, Tanahashi T (1993) Four tetrahydroisoquinoline-monoterpene glucosides from Cephaelis ipecacuanha. Phytochemistry 32(3):761-765. https://doi.org/10. 1016/S0031-9422(00)95167-7

Nakano T, Martín A (1976) Studies on the alkaloids of Palicourea fendleri. Planta Med 30(2):186-188. https://doi. org/10.1055/s-0028-1097715

Narine LL, Maxwell AR (2009) Monoterpenoid indole alkaloids from Palicourea crocea. Phytochem Lett 2(1):34-36. https://doi.org/10.1016/j.phytol.2008.10.007

Nascimento RRG, Monteiro JA, Pimenta ATA, Trevisan MTS, Braz-Filho R, de Souza EB, Silveira ER, Lima MAS (2015a) Novos flavonoides de Margaritopsis carrascoana com atividade antioxidante. Quím Nov 38(1):60-65. https://doi.org/10.5935/0100-4042.20140289

Nascimento RRG, Pimenta ATA, de Lima NP, Junior JRC, Costa-Lotufo LV, Ferreira EG, Tinoco LW, Braz-Filho R, Silveira ER, Lima MAS (2015b) New alkaloids from Margaritopsis carrascoana (Rubiaceae). J Braz Chem Soc 26(6):1152-1159. https://doi.org/10.5935/0103-5053. 20150079

Naves RF (2014) Estudo fitoquímico das folhas de Psychotria hoffmannseggiana Roem. \& Schult. (Rubiaceae). Master Thesis, Universidade Federal de Goiás, Goiás, Brazil, pp 1-212. http://repositorio.bc.ufg.br/tede/handle/tede/ 3612

Nepokroeff M, Bremer B, Sytsma KJ (1999) Reorganization of the genus Psychotria and tribe Psychotrieae (Rubiaceae) inferred from ITS and rbcL sequence data. Syst Bot 24(1):5-27. https://doi.org/10.2307/2419383

Nomura T, Quesada AL, Kutchan TM (2008) The new $\beta$-Dglucosidase in terpenoid-isoquinoline alkaloid biosynthesis in Psychotria ipecacuanha. $\mathrm{J}$ Biol Chem 283(50):34650-34659. https://doi.org/10.1074/jbc. M806953200

Nomura T, Kutchan TM (2010a) Three new $O$-methyltransferases are sufficient for all $O$-methylation reactions of ipecac alkaloid biosynthesis in root culture of Psychotria ipecacuanha. J Biol Chem 285(10):7722-7738. https://doi. org/10.1074/jbc.M109.086157

Nomura T, Kutchan TM (2010b) Is a metabolic enzyme complex involved in the efficient and accurate control of Ipecac alkaloid biosynthesis in Psychotria ipecacuanha? Plant Signal Behav 5(7):875-877. https://doi.org/10.4161/psb.5. 7.11901

O'Connor SE, Maresh JJ (2006) Chemistry and biology of monoterpene indole alkaloid biosynthesis. Nat Prod Rep 23(4):532-547. https://doi.org/10.1039/B512615K

de Oliveira CF (2015) Morfoanatomia, caracterização fitoquímica e avaliação das atividades biológicas de Psychotria fractistipula L.B. SM, Klein \& Delprete (Rubiaceae). Dissertação, Universidad Federal do Paraná, Curitiba. https://hdl.handle.net/1884/37389

Parry KP, Smith GF (1978) Quadrigemines-A and -B, two minor alkaloids of Hodgkinsonia frutescens F. Muell. J Chem Soc, Perkin Trans 1(12):1671-1682. https://doi.org/10. 1039/P19780001671

Paul JHA, Maxwell AR, Reynolds WF (2003) Novel bis(monoterpenoid) indole alkaloids from Psychotria bahiensis. J Nat Prod 66(6):752-754. https://doi.org/10.1021/ np020554a

Pimenta ATÁ, Braz-Filho R, Delprete PG, de Souza EB, Silveira ER, Lima MAS (2010a) Structure elucidation and NMR assignments of two unusual monoterpene indole alkaloids from Psychotria stachyoides. Magn Res Chem 48(9):734-737. https://doi.org/10.1002/mrc.2656

Pimenta ATÁ, Braz-Filho R, Delprete PG, de Souza EB, Silveira ER, Lima MAS (2010b) Unusual monoterpene indole alkaloids from Psychotria stachyoides Benth. Biochem Syst Ecol 38(4):846-849. https://doi.org/10.1016/j.bse. 2010.07.013

Pimenta ATÁ, Uchôa DE, Braz-Filho R, Silveira ER, Lima MAS (2011) Alkaloid and other chemical constituents from Psychotria stachyoides Benth. J Braz Chem Soc 22(11):2216-2219. https://doi.org/10.1590/S010350532011001100027

Pinheiro RP, Moraes MA, Santos BCS, Fabri RL, Del-VechioVieira G, Yamamoto CH, Araújo ALSM, Araújo ALA, Sousa OV (2018) Identification of compounds from Palicourea rigida leaves with topical anti-inflammatory potential using experimental models. Inflammopharmacology 26(4):1005-1016. https://doi.org/10.1007/s10787017-0415-3

Porto DD, Henriques AT, Fett-Neto AG (2009) Bioactive alkaloids from South American Psychotria and related 
species. Open Bioact Comp J 2:29-36. https://doi.org/10. 2174/1874847300902010029

Prachayasittikul S, Buraparuangsang P, Worachartcheewan A, Isarankura-Na-Ayudhya $\mathrm{C}$, Ruchirawat $\mathrm{S}$, Prachayasittikul V (2008) Antimicrobial and antioxidative activities of bioactive constituents from Hydnophytum formicarum Jack. Molecules 13(4):904-921. https://doi.org/10.3390/ molecules 13040904

Prachayasittikul S, Pingaew R, Yamkamon V, Worachartcheewan A, Wanwimolruk S, Ruchirawat S, Prachayasittikul V (2012) Chemical constituents and antioxidant activity of Hydnophytum formicarum Jack. Int J Pharmacol 8(5):440-444. https://doi.org/10.3923/ijp.2012.440.444

Queiroz GS, Luz ABG, dos Santos Nascimento MVP, Thomasi SS, Ferreira AG, Dalmarco EM, Brighente IMC (2017) Phytochemical study and anti-inflammatory effect of Psychotria stenocalyx (Rubiaceae). J App Pharm Sci 7(04):168-173. https://doi.org/10.7324/JAPS.2017.70425

Ramil RJD, Ramil MDI, Konno T, Murata T, Kobayashi K, Buyankhishig B, Agrupis SC, Sasaki K (2020) A new hexenoic acid glycoside with cytotoxic activity from the leaves of Psychotria luzoniensis. Nat Prod Res. https://doi. org/10.1080/14786419.2020.1765345

Rao H, Lai P, Gao Y (2017) Chemical composition, antibacterial activity, and synergistic effects with conventional antibiotics and nitric oxide production inhibitory activity of essential oil from Geophila repens (L.) I.M. Johnst. Molecules 22(9):1561 https://doi.org/10.3390/ molecules 22091561

Rasolonjanahary R, Sévenet T, Guéritte-Voegelein F, Kordon C (1995) Psycholeine, a natural alkaloid extracted from Psychotria oleoides, acts as a weak antagonist of somatostatin. Eur J Pharmacol 285(1):19-23. https://doi.org/10. 1016/0014-2999(95)00345-L

Razafimandimbison SG, Taylor CM, Wikström N, Pailler T, Khodabandeh A, Bremer B (2014) Phylogeny and generic limits in the sister tribes Psychotrieae and Palicoureeae (Rubiaceae): Evolution of schizocarps in Psychotria and origins of bacterial leaf nodules of the Malagasy species. Am J Bot 101(7):1102-1126. https://doi.org/10.3732/ajb. 1400076

Rédei D, Abdullah H, Hawariah A, Forgo P, Molnár J, Hohmann J (2005) Constituents and antiproliferative activity of the Malaysian plant Hydnophytum formicarium. Poster: 53rd Annual Congress of the Society for Medicinal Plant Research, Florence, Italy. https://doi.org/10.13140/RG.2.2. 32172.49288

Ribeiro MAS, Gomes CMB, Formagio ASN, Pereira ZV, Melo UZ, Basso EA, da Costa WF, Baldoqui DC, Sarragiotto MH (2016) Structural characterization of dimeric indole alkaloids from Psychotria brachybotrya by NMR spectroscopy and theoretical calculations. Tetrahedron Lett 57(12):1331-1334. https://doi.org/10.1016/j.tetlet.2016.02.040

Ripperger H (1982) Chimonanthin aus Palicourea domingensis. Pharmazie 37(12):867

Rivier L, Lindgren JE (1972) Ayahuasca, the South American hallucinogenic drink: An ethnobotanical and chemical investigation. Econ Bot 26(2):101-129

Robbrecht E (1975) Hymenocoleus, a new genus of Psychotrieae (Rubiaceae) from tropical Africa. Bull Jard Bot Nat Belg 45:273-300. https://doi.org/10.2307/3667482
Robbrecht E, Manen JF, (2006) The major evolutionary lineages of the coffee family (Rubiaceae, angiosperms). Combined analysis (nDNA and cpDNA) to infer the position of Coptosapelta and Luculia, and supertree construction based on rbcL, rps16, trnL-trnF and atpB-rbcL data. A new classification in two subfamilies, Cinchonoideae and Rubioideae. Syst Geogr Pl 76(1):85-145. https://www. jstor.org/stable/20649700

Roth A, Kuballa B, Cabalion P, Anton R (1985) Preliminary study of the alkaloids of Psychotria forsteriana. Planta Med 51(3):289. https://doi.org/10.1055/s-2007-969491

Roth A, Kuballa B, Bounthanh C, Cabalion P, Sévenet T, Beck JP, Anton R (1986) Cytotoxic activity of polyindoline alkaloids of Psychotria forsteriana (Rubiaceae). Planta Med 52(06):450-453. https://doi.org/10.1055/s-2007969251

Saad HEA, El-Sharkawy SH, Shier WT (1995) Biological activities of pyrrolidinoindoline alkaloids from Calycodendron milnei. Planta Med 61(4):313-316. https://doi. org/10.1055/s-2006-958090

Samulski GB, Gontijo DC, Moreira NC, Brandão GC, de Oliveira AB (2020) Dereplication of Palicourea sessilis ethanol extracts by UPLC-DAD-ESI-MS/MS discloses the presence of hydroxycinnamic acid amides and the absence of monoterpene indole alkaloids. Biochem Syst Ecol 92:104114. https://doi.org/10.1016/j.bse.2020.104114

Sandra UI, Ishmael AV, Raphael O, Cookey I, Sani G, Godfrey N, Edet EE (2018) Chromatographic assay, antimicrobial analysis and structural elucidation of bioactive compounds of Palicourea croceiodes leaves extract. Am J Biol Chem 5(2):6-14

Satari MH, Situmeang B, Yudha IP, Kurnia D (2019) Antibacterial diterpenoid against pathogenic oral bacteria of Streptococcus mutans ATCC 25175 isolated from Sarang Semut (Myrmecodia pendans). Jurnal Kimia Valensi 5(2):218-223 https://doi.org/10.15408/jkv.v5i2.8864

Schindler F, Fragner L, Herpell JB, Berger A, Brenner M, Tischler S, Bellaire A, Schönenberger J, Li W, Sun X, Schinnerl J, Brecker L, Weckwerth W (2021) Dissecting metabolism of leaf nodules in Ardisia crenata and Psychotria punctata. Front Mol Biosci 8:683671. https://doi. org/10.3389/fmolb.2021.683671

Schinnerl J, Orlowska EA, Lorbeer E, Berger A, Brecker L (2012) Alstrostines in Rubiaceae: Alstrostine A from Chassalia curviflora var. ophioxyloides and a novel derivative, rudgeifoline from Rudgea cornifolia. Phytochem Lett 5(3):586-590. https://doi.org/10.1016/j. phytol.2012.05.019

Schmidt AW, Reddy KR, Knölker HJ (2012) Occurrence, biogenesis, and synthesis of biologically active carbazole alkaloids. Chem Rev 112(6):3193-3328. https://doi.org/ $10.1021 / \mathrm{cr} 200447 \mathrm{~s}$

Setzer WN, Noletto JA, Haber WA (2006) Chemical composition of the floral essential oil of Psychotria eurycarpa from Monteverde, Costa Rica. J Essent Oil Bear Plants 9(1):28-31. https://doi.org/10.1080/0972060X.2006. 10643466

Shamma M (1972) Emetine and related alkaloids. In: Organic Chemistry 25: The isoquinoline alkaloids. Academic Press, New York, pp 426-457. https://doi.org/10.1016/B978-012-638250-1.50027-7 
Sieber S, Carlier A, Neuburger M, Grabenweger G, Eberl L, Gademann K (2015) Isolation and total synthesis of kirkamide, an aminocyclitol from an obligate leaf nodule symbiont. Angew Chem 127(27):8079-8081. https://doi. org/10.1002/anie.201502696

Silva KTD, Smith GN, Warren KEH (1971) Stereochemistry of strictosidine. Chem Commun 16:905-907. https://doi.org/ 10.1039/C29710000905

Simões-Pires CA, Farias FM, Marston A, Queiroz EF, Chaves CG, Henriques AT, Hostettmann K (2006) Indole monoterpenes with antichemotactic activity from Psychotria myriantha: Chemotaxonomic significance. Nat Prod Commun 1(12):1101-1106. https://doi.org/10.1177/ 1934578X0600101206

Snow DW (1981) Tropical frugivorous birds and their food plants: a World survey. Biotropica 13(1):1-14. https://doi. org/10.2307/2387865

Soares DBS, Duarte LP, Cavalcanti AD, Silva FC, Braga AD, Lopes MT, Takahashi JA, Vieira-Filho SA (2017) Psychotria viridis: Chemical constituents from leaves and biological properties. An Acad Bras Ciênc 89(2):927-938. https://doi.org/10.1590/0001-3765201720160411

Sohmer SH, Davis AP (2007) The genus Psychotria (Rubiaceae) in the Philippine Archipelago. Sida. Bot Misc 37:1-247

Solis PN, Wright CW, Gupta MP, Phillipson JD (1993) Alkaloids from Cephaelis dichroa. Phytochemistry 33(5):1117-1119. https://doi.org/10.1016/00319422(93)85033-N

Solis PN, Lang'at C, Gupta MP, Kirby GC, Warhurst DC, Phillipson JD (1995) Bio-active compounds from Psychotria camponutans. Planta Med 61(1):62-65. https://doi. org/10.1055/s-2006-958001

Solis PN, Ravelo AG, Palenzuela JA, Gupta MP, González A, Phillipson JD (1997) Quinoline alkaloids from Psychotria glomerulata. Phytochemistry 44(5):963-969. https://doi. org/10.1016/S0031-9422(96)00583-3

Soobrattee MA, Bahorun T, Thaunoo P (2005) Characterization of the phenolic profile of endemic Mauritian Chassalia species and assessment of their antioxidant activities. In: Lalouette JA, Bheenick KJ, Nundalallee C (eds) Proceedings of the Seventh Meeting of Agricultural Scientists, Réduit, Mauritius, 4-6 May 2005. Food and Agricultural Research Council, Réduit, Mauritius, pp 13-21

Sosa Moreno A (2011) Etude phytochimique de plantes médicinales des Andes Vénézuéliennes: Palicourea demissa Standl. (Rubiaceae) et Hydrocotyle umbellata L. (Umbelliferae). Doctoral thesis, Université Bordeaux http://www.theses.fr/2011BOR14274/document

Steyermark JA (1972) The botany of the Guayana HighlandsPart IX. Rubiaceae Mem New York Bot Gard 23:227-832

Stuart KL, Woo-Ming RB (1974) Palicourea alkaloids: the structure of palinine. Tetrahedron Lett 15(44):3853-3856. https://doi.org/10.1016/S0040-4039(01)92027-1

Stuart KL, Woo-Ming RB (1975) Vomifoliol in Croton and Palicourea species. Phytochemistry 14(2):594-595. https://doi.org/10.1016/0031-9422(75)85146-6

Takayama H, Mori I, Kitajima M, Aimi N, Lajis NH (2004) New type of trimeric and pentameric indole alkaloids from Psychotria rostrata. Org Lett 6(17):2945-2948. https://doi. org/10.1021/ol048971x
Tan MA, Eusebio JA, Alejandro GJD (2012) Chemotaxonomic implications of the absence of alkaloids in Psychotria gitingensis. Biochem Syst Ecol 45:20-22. https://doi.org/ 10.1016/j.bse.2012.07.016

Tan MA, Panghulan GFM, Uy MM, Takayama H (2014) Chemical constituents from Psychotria cadigensis and their chemotaxonomic relevance. Am J Essent Oil Nat Prod 1(4):18-19

Taylor CM (1996) Overview of the Psychotrieae (Rubiaceae) in the Neotropics. Opera Bot Belg 7:261-270

Taylor CM (2001) Overview of the neotropical genus Notopleura (Rubiaceae: Psychotrieae), with the description of some new species. Ann Missouri Bot Gard 88(3):478-515. https://doi.org/10.2307/3298587

Taylor CM (2019a) Rubiacearum Americanarum Magna Hama Pars XLIV: Review of the Palicourea pilosa group, with some new species and a new subspecies (Palicoureeae). Novon 27(2):102-130. https://doi.org/10.3417/2018316

Taylor CM (2019b) Rubiacearum Americanarum Magna Hama Pars XLV: More new species and taxonomic changes in Palicourea (Rubiaceae, Palicoureeae) and Psychotria subg. Heteropsychotria. Novon 27(3):165-195. https://doi. org/10.3417/2019387

Taylor CM (2020) Overview of Psychotria in Madagascar (Rubiaceae, Psychotrieae), and of Bremekamp's foundational study of this group. Candollea 75(1):51-70. https:// doi.org/10.15553/c2020v751a5

Taylor CM, Gereau RE (2013) The genus Carapichea (Rubiaceae, Psychotrieae). Ann Missouri Bot Gard 99(1):100-127. https://www.jstor.org/stable/42703711

Taylor CM, Schmidt GRE (2020) Some distinctive new species of Psychotria from Madagascar (Rubiaceae, Psychotrieae). Candollea 75(2):159-182. https://doi.org/10.15553/ c2020v752a1

Taylor CM, Lorence DH, Gereau RE (2010) Rubiacearum Americanarum Magna Hama Pars XXV: the nocturnally flowering Psychotria domingensis-Coussarea hondensis group plus three other Mesoamerican Psychotria species transfer to Palicourea. Novon 20(4):481-492. https://doi. org/10.3417/2009124

Taylor CM, Hollowell VC (2016) Rubiacearum Americanarum Magna Hama Pars XXXV: The new group Palicourea sect. Nonatelia, with five new species (Palicoureeae). Novon 25(1):69-110. https://doi.org/10.3417/2015012

Taylor CM, Razafimandimbison SG, Barrabé L, Jardim JG, Barbosa MRV (2017) Eumachia expanded, a pantropical genus distinct from Psychotria (Rubiaceae, Palicoureeae). Candollea 72(2):289-318. https://doi.org/10.15553/c2017v722a6

Taylor CM (2005) Margaritopsis (Rubiaceae, Psychotrieae) in the Neotropics. Syst Geogr Pl 75(2):161-177. http://www. jstor.org/stable/3668574

Taylor CM (2014) Rubiaceae. In: Hammel BE, Grayum MH, Herrera C, Zamora N (eds) Manual de Plantas de Costa Rica. Vol. VII. Monogr Syst Bot Missouri Bot Gard 129:464-779

Taylor CM (2015a) Rubiacearum Americanarum Magna Hama XXXIII: The new group Palicourea sect. Didymocarpae with four new species and two new subspecies (Palicoureeae). Novon 23(4):452-478. https://doi.org/10.3417/ 2012003 
Taylor CM (2015b) Rubiacearum Americanarum Magna Hama Pars XXXIV: The new group Palicourea sect. Tricephalium with eight new species and a new subspecies (Palicoureeae). Novon 24(1):55-95. https://doi.org/10.3417/ 2015001

Taylor CM (2017) Rubiacearum Americanarum Magna Hama XXXVII: The new group Palicourea sect. Chocoanae of the Chocó biogeographic region, with two new species (Palicoureeae). Novon 25(3):322-342. https://doi.org/10. $3417 / 2016002$

Taylor CM (2018) Rubiacearum Americanarum Magna Hama Pars XXXVIII: A new circumscription of Palicourea sect. Bracteiflorae, an Andean radiation with several new species (Palicoureeae). Novon 26(1):66-138. https://doi. org/10.3417/2017036

Tran PH, Le VD, Do TH, Nguyen TL, Nguyen PT, Nguyen TT, Nguyen TD (2019) Anti-inflammatory constituents from Psychotria prainii H. Lév Nat Prod Res 33(5):695-700. https://doi.org/10.1080/14786419.2017.1408095

Uzor PF (2016) Recent developments on potential new applications of emetine as anti-cancer agent. EXCLI Journal 15:323-328. https://doi.org/10.17179/excli2016-280

Valdevite M, Bertoni BW, Contini SHT, de Castro FS, Pereira AMS (2016) Accumulation of loganin by genotypes of Palicourea rigida and related differential gene expression as determined by cDNA-SRAP. Plant Cell, Tissue Organ Cult 125(3):445-456. https://doi.org/10.1007/s11240-0160959-8

Valverde J, Tamayo G, Hesse M (1999) $\beta$-Carboline monoterpenoid glucosides from Palicourea adusta. Phytochemistry 52(8):1485-1489. https://doi.org/10.1016/S00319422(99)00215-0

van de Santos L, Fett-Neto AG, Kerber VA, Elisabetsky E, Quirion JC, Henriques AT (2001) Indole monoterpene alkaloids from leaves of Psychotria suterella Müll. Arg. (Rubiaceae). Biochem Syst Ecol 29(11):1185-1187. https://doi.org/10.1016/S0305-1978(01)00059-X

Van Elst D, Nuyens S, Van Wyk B, Verstraete B, Dessein S, Prinsen E (2013) Distribution of the cardiotoxin pavettamine in the coffee family (Rubiaceae) and its significance for gousiekte, a fatal poisoning of ruminants. Plant Physiol Biochem 67:15-19. https://doi.org/10.1016/j.plaphy.2013. 02.022

Vencato I, da Silva FM, de Oliveira CMA, Kato L, Tanaka CMA, da Silva CC, Sabino JR (2006) Vallesiachotamine Acta Crystallogr E 62:o429-o431. https://doi.org/10.1107/ S1600536805041553

Verotta L, Pilati T, Tatò M, Elisabetsky E, Amador TA, Nunes DS (1998) Pyrrolidinoindoline alkaloids from Psychotria colorata. J Nat Prod 61(3):392-396. https://doi.org/10. 1021/np9701642

Verotta L, Peterlongo F, Elisabetsky E, Amador TA, Nunes DS (1999) High-performance liquid chromatography-diode array detection-tandem mass spectrometry analyses of the alkaloid extracts of Amazon Psychotria species. J Chromatogr A 841(2):165-176. https://doi.org/10.1016/S00219673(99)00298-8

Wang Y, Zhou J (1999) Chemical constituents of curvedflower chasalis bark (Chasalis curviflora). Zhongcaoyao 30(9):644-645
Wang YH, Samoylenko V, Tekwani BL, Khan IA, Miller LS, Chaurasiya ND, Rahman MM, Tripathi LM, Khan SI, Joshi VC, Wigger FT (2010) Composition, standardization and chemical profiling of Banisteriopsis caapi, a plant for the treatment of neurodegenerative disorders relevant to Parkinson's disease. J Ethnopharmacol 128(3):662-671. https://doi.org/10.1016/j.jep.2010.02.013

Wang K, Zhou X-Y, Wang Y-Y, Li M-M, Li Y-S, Peng L-Y, Cheng X, Li Y, Wang Y-P, Zhao Q-S (2011) Macrophyllionium and macrophyllines $\mathrm{A}$ and $\mathrm{B}$, oxindole alkaloids from Uncaria macrophylla. J Nat Prod 74(1):12-15. https://doi.org/10.1021/np1004938

Wiegrebe W, Kramer WJ, Shamma M (1984) The emetine alkaloids. J Nat Prod 47(3):397-408. https://doi.org/10. 1021/np50033a001

Woo-Ming RB, Stuart KL (1975) Calycanthine from Palicourea alpina. Phytochemistry 14(11):2529. https://doi.org/10. 1016/0031-9422(75)80394-3

Yang H, Zhang H, Yang C, Chen Y (2016) Chemical constituents of plants from the genus Psychotria. Chem Biodiv 13(7):807-820. https://doi.org/10.1002/cbdv.201500259

Yang HM, Zhang HM, Zhang YK, Liao PN, Chen YG (2018) Compounds from the twigs and leaves of Psychotria prainii. Chem Nat Compd 54(1):178-180. https://doi.org/ 10.1007/s10600-018-2289-Z

Ye H-C, Zheng X-H, Wang Y-M, Peng G-T, Su X-J, Lei L-F, Zhang C-X (2014) Saponins from the stem of Psychotria sp. Zhongshan Daxue Xuebao 53(1):93-97

Young MCM, Araújo AR, da Silva CA, Lopes MN, Trevisan LMV (1998) Triterpenes and saponins from Rudgea viburnioides. J Nat Prod 61(7):936-938. https://doi.org/10. 1021/np9704617

Zappi DC (2003) Revision of Rudgea (Rubiaceae) in southeastern and southern Brazil. Kew Bull. 58(3):513-596. https://doi.org/10.2307/4111145

Zhang C-X, He X-X, Guan S-Y, Zhong Y, Lin C-Z, Xiong T-Q, Zhu C-C (2012) New sphingolipid psychotramide A-D from the stem of Psychotria sp. Nat Prod Res 26(20):1864-1868. https://doi.org/10.1080/14786419. 2011.617747

Zhang C-X, Zhang D-M, Chen M-F, Guan S-Y, Yao J-H, He X-X, Lei L-F, Zhong Y, Wang Z-F, Ye W-C (2013) Antiproliferative triterpenoid saponins from the stem of Psychotria sp. Planta Med 79(11):978-986. https://doi.org/ 10.1055/s-0032-1328650

Zhang C-X, Peng G-T, He X-X, Lin C-Z, Xiong T-Q, Deng J-W, Zhao Z-X, Zhu C-C (2010) Chemical constituents of Psychotria sp. (I). Zhongshan Daxue Xuebao 49(4):22-25

Zhou H, He H-P, Wang Y-H, Hao X-J (2010) A new dimeric alkaloid from the leaf of Psychotria calocarpa. Helv Chim Acta 93(8):1650-1652. https://doi.org/10.1002/hlca. 200900439

Zhou BD, Zhang XL, Niu HY, Guan CY, Liu YP, Fu YH (2018) Chemical constituents from stems and leaves of Psychotria serpens. Zhongguo Zhong Yao Za Zhi 43(24):4878-4883. https://doi.org/10.19540/j.cnki.cjcmm.20180912.003

Publisher's Note Springer Nature remains neutral with regard to jurisdictional claims in published maps and institutional affiliations. 